\title{
Model Reference Adaptive Control of Distributed Parameter Systems
}

\author{
Michael Böhm * \\ FB Mathematik \\ Humboldt-Universität zu Berlin \\ 10099 Berlin Germany
M. A. Demetriou $+\S$
Center for Research in Scientific Computation
Department of Mathematics
North Carolina State University
Raleigh, North Carolina 27695 USA \\ Simeon Reich $\ddagger$ \\ Department of Mathematics \\ University of Southern California \\ Los Angeles, California 90089-1113 USA \\ and \\ Department of Mathematics \\ The Technion-Israel Institute of Technology \\ 32000 Haifa, Israel \\ I.G. Rosen $\S$ \\ Center for Applied Mathematical Sciences \\ Department of Mathematics \\ University of Southern California \\ Los Angeles, California 90089-1113 USA
}

February 13, 1995

\begin{abstract}
A model reference adaptive control law is defined for nonlinear distributed parameter systems. The reference model is assumed to be governed by a strongly coercive linear operator defined with respect to a Gelfand triple of reflexive Banach and Hilbert spaces. The resulting nonlinear closed loop system is shown to be well posed. The tracking error is shown to converge to zero, and regularity results for the control input and the output are established. With an additional richness, or persistence of excitation assumption, the parameter error is shown to converge to zero as well. A finite dimensional approximation theory is developed. Examples involving both first (parabolic) and second (hyperbolic) order systems and linear and nonlinear systems are discussed, and numerical simulation results are presented.
\end{abstract}

\footnotetext{
${ }^{*}$ Supported in part by DFG.

${ }^{\dagger}$ Supported in part by the Air Force Office of Scientific Research under grant AFOSR F49620-93-1-0198, and in part by NASA under grant NAG-1-1600.

${ }^{\ddagger}$ Supported in part by the Fund for the Promotion of Research at the Technion.

${ }^{\S}$ Supported in part by the Air Force Office of Scientific Research under grant AFOSR 91-0076.
} 


\section{Introduction}

In this paper we develop a model reference adaptive control (MRAC) scheme for rather broad classes of, in general, nonlinear distributed parameter systems. By a distributed parameter system we mean one in which the state space is infinite dimensional such as occurs in the case of partial and functional (i.e. hereditary and neutral) differential equations. In the context of finite dimensional systems, model reference adaptive control is one of the standard approaches taken in designing a control law for a plant with unknown parameters. A complete description and analysis of a variety of approaches to MRAC can be found in any one of a number of standard texts on adaptive control (see, for example, [2], [13], [29], and [34]). The objective of a MRAC scheme is to determine a feedback control law which forces the state of the plant to asymptotically track the state of a given reference model. At the same time, the unknown parameters in the plant model are estimated and used to update the control law. Typically, the resulting closed loop system consisting of the plant, the reference model, and the estimator, will be nonlinear. This is true even if the underlying plant and reference models, and the estimator, are linear. The nonlinearity arises in the coupling. Consequently, the scheme requires a careful stability analysis to ensure that all signals (both input and output) remain, in some sense, bounded. It is also desirable, although not necessarily essential, that some sort of parameter convergence be achieved.

The focus of the effort we describe here is the extension of one approach to finite dimensional MRAC to infinite dimensional systems. We consider nonlinear plants with the restriction that their dependence on the unknown parameters be affine. The operator describing the dynamics of the reference model is assumed to be linear and strongly $V$-coercive (in a Gelfand triple setting). The parameter space can be either finite or infinite dimensional, and the estimator dynamics for the unknown parameters, which are linear, are chosen in a fashion which renders the closed loop error equations skew-self-adjoint. This is analogous to what is done in finite dimensions and has the effect of facilitating both tracking error and parameter convergence by forcing the time derivative of a certain energy functional to be negative semi-definite. We establish the global well-posedness of the closed loop system via two different approaches. First we argue existence of a local solution and then its continuation by treating the closed loop system as semilinear (i.e. a nonlinear perturbation of a linear system) with the linear component of the dynamics being the infinitesimal generator of an analytic semigroup. The second approach involves the application of an abstract version of the Implicit Function Theorem to obtain a global solution when the initial tracking and parameter error are sufficiently small. Using an analog of Barbălat's lemma, we establish that the tracking error approaches zero asymptotically. We also establish regularity results for both the input and output signals. In particular, we establish a boundedness result for the control signal. With the additional assumption of persistence of excitation, a richness condition on the plant, reference model, and input reference signal, we establish parameter convergence. Since the reference model and estimator are, in general, infinite dimensional, implementation requires some form of finite dimensional approximation. Consequently, we develop an abstract finite dimensional approximation and convergence theory. Finally, we illustrate the application of our general theory on a number of examples involving distributed parameter systems of various types (e.g. parabolic, hyperbolic, linear, nonlinear, etc.).

One drawback of our approach is that it requires (as do the analogous finite dimensional 
schemes, see, for example, [29]) measurement of the full state, and distributed input. Eliminating either of these restrictions represents a formidable challenge. For example, if only a partial state measurement is available, a coupled adaptive observer would be required. The corresponding analysis would be significantly more complicated than the already rather technical arguments we present here. We are currently looking at the extension of our treatment here to include partial measurements and finite dimensional input.

Our effort here is related to our earlier treatment of adaptive identification for distributed parameter systems in [6], [7], and [8]. In fact, we employ the same estimator here to identify the unknown parameters in the plant, and the arguments used below (infinite dimensional analogs of the finite dimensional theory presented in [28] and [30]) to demonstrate the asymptotic convergence of the tracking and parameter error to zero, are similar to the ones employed to establish state and parameter convergence for the identification schemes. However, in the case of the identification schemes, the resulting estimator equations are linear. In the case of MRAC, the resulting closed loop system is nonlinear. Consequently, certain aspects of the analysis, in particular, those dealing with the well-posedness of the closed loop system and the convergence of the finite dimensional approximation, are more delicate. Other related treatments of on-line or adaptive identification for distributed parameter systems can be found in [1], [5], [15], [16], [17], and [36].

Recently there has been some attention given to the adaptive control of distributed parameter systems. First, with respect to approaches other than model reference, indirect adaptive control algorithms for a class of infinite dimensional stochastic evolution equations have been developed by Duncan, Pasik-Duncan, and their co-workers in a recent series of papers [10], [11], [12], and [31]. Their approach involves the use of a least squares based estimator together with a linear quadratic control design. Parameter convergence together with a continuous dependence result (with respect to the unknown parameters) for the solutions to the operator algebraic Riccati equations yield convergence of the adaptive control law to the nonadaptive optimal LQ controller. Also, Kobayashi in [20], [21], [22], [23], and [24] has proposed a number of direct schemes based upon an input/output formulation. His approach is primarily directed towards the case of unknown input and/or output operators (i.e. the $B$ and $C$ operators) and places a number of restrictions on the $A$ operator (for example, that it be self-adjoint, its eigenvalues be known, that only a finite number of modes be unstable, etc.).

In [39] and [40] a finite dimensional approach to model reference adaptive control based upon the so-called command generator tracker, is extended to infinite dimensions. The command generator tracker theory deals with the problem of a mis-match in the dimensionality of the plant and the reference model by assuming that there is an infinite dimensional system that is input/output equivalent to the reference model. The authors establish closed loop stability (and robustness properties) via a Lyapunov argument (which in infinite dimensions must be done with care) under a number of rather technical assumptions.

In a recent effort by Hong and Bentsman [18] the authors consider the model reference adaptive control of linear parabolic partial differential equations. Formally, and only formally, with respect to the choice of the control and parameter adaptation laws, and the argument establishing state error convergence, their approach and ours are the same. Of course since both studies involve model reference adaptive control, the desired objectives (i.e. the convergence of the state tracking and parameter errors to zero with closed loop input and output signals remaining, in some sense, bounded) both here and in [18] are the same. Both treatments 
assume full state measurement and distributed input. However, in all other respects, the two treatments have nothing in common. In particular, the results in [18] apply only to plants and reference models which are linear parabolic partial differential equations with Dirichlet boundary conditions. Other portions of their analysis are only carried out under additional restrictions such as one dimensional spatial domain. On the other hand, in the present treatment, the plant and reference model are independent and must only satisfy a few relatively mild abstract assumptions. In particular, we consider general nonlinear plants and require only that the reference model dynamics be strongly $V$-coercive (in a Gelfand triple sense). In [18] the authors make the rather restrictive assumption that the reference signal and the plant and reference model parameters are analytic. We again require only minimal abstract assumptions on the parameter and reference signal spaces. The primary reason for these differences is that our approach and analysis is entirely functional analytic/abstract operator theoretic in nature, whereas in [18] the treatment has a more classical flavor. In [18] the authors provide a parameter convergence result based upon a very different notion of persistence of excitation than the one we define here. They seem to establish that if the spatial gradient of the plant is zero only on a set of measure zero (their definition of persistence of excitation) and the state tracking error is identically zero then the parameter error is identically zero. It is unclear to these authors why this result is useful and, moreover, what it has to do with the usual notion of parameter convergence. On the other hand, our definition of persistence of excitation for infinite dimensional systems is the natural extension of the analogous result for finite dimensional systems as given in, for example, [28], [29] and [30]. With this assumption, we are able to establish that the parameter space norm of the parameter error tends to zero asymptotically. Hong and Bentsman also provide state tracking error and parameter convergence results (exponential stability of the trivial solution to the error equations) via an averaging technique. However, it seems that these results, as is also the case for our parameter convergence result, can only be applied (i.e. the requisite hypotheses checked) in very simple cases. For example, plants and reference models that consist of one or two dimensional heat equations with constant coefficients. Finally, we have developed a finite dimensional approximation theory and have established associated convergence results. This has not been included in [18].

An outline of the remainder of the paper is as follows. In Section 2 we define the plant, reference model, and estimator, we derive the closed loop system and establish well posedness. In Section 3 we establish the convergence of the tracking error to zero, we define persistence of excitation and demonstrate parameter convergence. The finite dimensional approximation and convergence theory is discussed in Section 4, and examples and the results of our numerical studies are presented in Section 5.

\section{The Model Reference Adaptive Control Problem}

Let $\{H,\langle\cdot, \cdot\rangle,|\cdot|\}$ be a Hilbert space over $\mathbf{R}$, and let $\{V,\|\cdot\|\}$ be a reflexive Banach space over $\mathbf{R}$ which is densely and continuously embedded in $H$. Then (see, for example, [26], [37], or [38])

$$
V \hookrightarrow H \hookrightarrow V^{*},
$$

with the embeddings dense and continuous where $V^{*}$ denotes the continuous dual of $V$. Let $\|\cdot\|_{*}$ denote the usual norm on $V^{*}$, and let $K>0$ be such that

$$
|\varphi| \leq K\|\varphi\|, \quad \varphi \in V \text {. }
$$


Let $\hat{V}^{*}$ be a subspace of $V^{*}$, and let $\left\{Q,\langle\cdot, \cdot\rangle_{Q},|\cdot|_{Q}\right\}$ be a real Hilbert space.

For each $q \in Q$, let $A_{1}(q): V \rightarrow V^{*}$ be an, in general, nonlinear operator, and for $q \in Q$, let $\operatorname{Dom}\left(A_{1}(q)\right)=\left\{\varphi \in V: A_{1}(q) \varphi \in H\right\}$. Also, we let $A_{2}: V \rightarrow V^{*}$ be an, in general, nonlinear operator, and we make the following standing assumptions.

(A1) ( $V-V^{*}$-Boundedness) There exist $\alpha_{1}, \alpha_{2}>0$ such that

$$
\left|\left\langle A_{1}(q) \varphi, \psi\right\rangle\right| \leq \alpha_{1}|q|_{Q}\|\varphi\|\|\psi\|, \quad \varphi, \psi \in V, q \in Q .
$$

and

$$
\left|\left\langle A_{2} \varphi, \psi\right\rangle\right| \leq \alpha_{2}\|\varphi\|\|\psi\|, \quad \varphi, \psi \in V .
$$

(A2) ( $Q$-Linearity) For each $\varphi \in V$, the map $q \rightarrow A_{1}(q) \varphi$ from $Q$ into $V^{*}$ is linear.

For each $q \in Q$, let $A(q): V \rightarrow V^{*}$ be given by

$$
A(q) \varphi=A_{1}(q) \varphi+A_{2} \varphi, \quad \varphi \in V .
$$

We are interested in adaptively controlling the nonlinear plant given by

$$
\begin{aligned}
D_{t} u(t)+A(\bar{q}) u(t) & =f(t), \quad \text { a.e. } t>0, \\
u(0) & =u_{0},
\end{aligned}
$$

where $\bar{q} \in Q$ is unknown, $u_{0} \in H$, the operator $A(\bar{q})$ is given by (2.2) with $q=\bar{q}$, and the control input $f$ is assumed to satisfy $f \in L_{2}\left(0, T ; V^{*}\right)$ for all $T>0$ with $f(t) \in \hat{V}^{*}$, a.e. $t>0$. We assume minimally, that the system $(2.3),(2.4)$ is well-posed in at least some sense. That is, we assume that for sufficiently regular initial data, $u_{0}$, and input, $f$, there exists a weak solution. More precisely, we assume that for each $T>0$, and each $u_{0} \in H$ and $f \in L_{2}\left(0, T ; V^{*}\right)$ sufficiently regular, there exists a unique $V$-valued function $u$ which is $V^{*}$-absolutely continuous on $(0, T)$,

$$
u \in C(0, T ; H) \cap L_{2}(0, T ; V), \quad D_{t} u \in L_{2}\left(0, T ; V^{*}\right),
$$

and which satisfies

$$
\begin{gathered}
\left\langle D_{t} u(t), \varphi\right\rangle+\langle A(\bar{q}) u(t), \varphi\rangle=\langle f(t), \varphi\rangle, \quad \varphi \in V, \text { a.e. } t>0, \\
u(0)=u_{0} .
\end{gathered}
$$

Theorem III.2.6 in [4] provides sufficient conditions for the existence of such a solution. Indeed, we would require that the operator $A(\bar{q})$ be hemicontinuous (i.e. $\lim _{\lambda \rightarrow 0}\langle A(\bar{q})\{\varphi+\lambda \psi\}-$ $A(\bar{q}) \varphi, \chi\rangle=0, \chi \in V$, for any $\varphi, \psi \in V$ ), monotone (i.e. $\langle A(\bar{q}) \varphi-A(\bar{q}) \psi, \varphi-\psi\rangle \geq 0$, for all $\varphi, \psi \in V$ ), bounded (i.e. there exists $\alpha>0$ for which $\|A(\bar{q}) \varphi\|_{*} \leq \alpha\{1+\|\varphi\|\}$, for all $\varphi \in V$ ) and coercive (i.e. there exist $\rho>0$ and $\sigma \in \mathbf{R}$ for which $\langle A(\bar{q}) \varphi, \varphi\rangle \geq \rho\|\varphi\|^{2}+\sigma$, for all $\varphi \in V$ ).

We are interested in designing a model reference adaptive controller for the plant, or system, $(2.3),(2.4)$. That is, we wish to find a control input $f$ in feedback form which forces the state of the unknown plant, $u$, to track the state of a given linear reference model,

$$
\begin{gathered}
\left\langle D_{t} v(t), \varphi\right\rangle+\left\langle A_{0} v(t), \varphi\right\rangle=\langle g(t), \varphi\rangle, \quad \varphi \in V, \text { a.e. } t>0, \\
v(0)=v_{0},
\end{gathered}
$$

where $v_{0} \in H$, the input reference signal $g$ is assumed to satisfy $g \in L_{2}\left(0, T ; V^{*}\right)$, for all $T>0$, with $g(t) \in \hat{V}^{*}$, a.e. $t>0$, and the operator $A_{0} \in \mathcal{L}\left(V, V^{*}\right)$ is assumed to satisfy the following conditions. 
(A3) $\left(V-V^{*}\right.$-Boundedness) There exists $\alpha_{0}>0$ such that

$$
\left|\left\langle A_{0} \varphi, \psi\right\rangle\right| \leq \alpha_{0}\|\varphi\|\|\psi\|, \quad \varphi, \psi \in V .
$$

(A4) ( $V$-Coercivity) There exists $\rho_{0}>0$ for which

$$
\left\langle A_{0} \varphi, \varphi\right\rangle \geq \rho_{0}\|\varphi\|^{2}, \quad \varphi \in V .
$$

(A5) ( $\hat{V}^{*}$-Range) For all $q \in Q$ we have $\mathcal{R}\left(A(q)-A_{0}\right) \subset \hat{V}^{*}$.

It is well known (see, for example, [26], [37], or [38]) that Assumptions (A3) and (A4) are sufficient to conclude that the system $(2.7),(2.8)$ admits a unique solution $v$ satisfying $v \in$ $C(0, T ; H) \cap L_{2}(0, T ; V)$ with $D_{t} v \in L_{2}\left(0, T ; V^{*}\right)$, for all $T>0$. Let $D_{0}=\operatorname{Dom}\left(A_{0}\right)=\{\varphi \in V$ : $\left.A_{0} \varphi \in H\right\}$. Then assumptions $(A 3)$ and $(A 4)$ also imply (see, for example, [32], [38], or [37]) that the operator $-A_{0}$ restricted to the subspace $D_{0}$ is the infinitesimal generator of an analytic semigroup, $\left\{T_{0}(t): t \geq 0\right\}$, of bounded linear operators on $H$. It can also be shown (see [38]) that the operator $-A_{0}$ is the infinitesimal generator of an analytic semigroup on $V^{*}$, and that appropriately restricted, $-A_{0}$ generates an analytic semigroup on $V$ (see [3]). Recalling (2.1), it follows that

$$
\left|T_{0}(t) \varphi\right| \leq e^{-\rho_{0} K^{-2} t}|\varphi|, \quad \varphi \in H,
$$

and

$$
\left\|T_{0}(t) \varphi\right\| \leq M e^{-\rho_{0} K^{-2} t}\|\varphi\|, \quad \varphi \in V
$$

for some $M>0$. The solution to the initial value problem $(2.7),(2.8)$ is given by

$$
v(t)=T_{0}(t) v_{0}+\int_{0}^{t} T_{0}(t-s) g(s) d s, \quad t \geq 0 .
$$

The primary motivation for the inclusion of Assumption (A5) is to allow us to apply our abstract framework to second order systems (i.e. abstract wave equations and the like). The relevance of Assumption ( $A 5)$ in this regard will become clearer when we discuss an example involving the control of a one dimensional wave equation in Section 5 below.

We have the following regularity result for the reference model, (2.7),(2.8).

Theorem 2.1 For the reference model given by (2.7),(2.8), we have the following results.

(i) If $g \in L_{\infty}(0, \infty ; H)$ then $v \in L_{\infty}(0, \infty ; H)$.

(ii) If $g \in L_{\infty}(0, \infty ; V)$ and $v_{0} \in V$, then $v \in L_{\infty}(0, \infty ; V)$.

(iii) If $g \in L_{2}\left(0, \infty ; V^{*}\right)$ then $v \in L_{\infty}(0, \infty ; H) \cap L_{2}(0, \infty ; V)$.

(iv) If $g \in L_{2}(0, \infty ; H)$ is Hölder continuous, i.e.

$$
|g(t)-g(s)| \leq C|t-s|^{\rho}, \quad 0 \leq t, s,<\infty,
$$

for some $C>0$ and $\rho \in(0,1]$, and $v_{0} \in V$, and if the operator $A_{0}$ is symmetric in the sense that

$$
\left\langle A_{0} \varphi, \psi\right\rangle=\left\langle A_{0} \psi, \varphi\right\rangle, \quad \varphi, \psi \in V,
$$

then $v \in L_{\infty}(0, \infty ; V), v(t) \in D_{0}$, a.e. $t>0$, and $A_{0} v \in L_{2}(0, \infty ; H)$. 
Proof. Statements (i) and (ii) follow immediately from (2.9), (2.10), and (2.11). To verify (iii), for almost every $t>0$ we have that

$$
\begin{aligned}
\frac{1}{2} D_{t}|v(t)|^{2} & =\left\langle-A_{0} v(t)+g(t), v(t)\right\rangle \\
& \leq-\rho_{0}\|v(t)\|^{2}+\left\|\left.g(t)\right|_{*}\right\| v(t) \| \\
& \leq-\left.\frac{\rho_{0}}{2}\left\|\left.v(t)\right|^{2}+\frac{1}{2}\right\| g(t)\right|_{*} ^{2} .
\end{aligned}
$$

Integrating both sides of the estimate (2.14) from 0 to $t$, it follows that

$$
|v(t)|^{2}+\int_{0}^{t}\|v(s)\|^{2} d s \leq\left|v_{0}\right|^{2}+\mid g \|_{L_{2}\left(0, \infty ; V^{*}\right)}^{2}, \quad t>0 .
$$

from which the result is immediately obtained.

To verify $(i v)$, first note that $A_{0}: D_{0} \subset H \rightarrow H$ is positive definite and self adjoint. It follows that the square root of $A_{0}, A_{0}^{\frac{1}{2}}$, can be defined with $\operatorname{Dom}\left(A_{0}^{\frac{1}{2}}\right)=V$ (see, for example, [38]). Moreover, for $\varphi \in V,\|\varphi\|_{0}=\left|A_{0}^{\frac{1}{2}} \varphi\right|$ defines a norm on $V$ and, by Assumptions $(A 3)$ and (A4), we have that

$$
\rho_{0}\|\varphi\|^{2} \leq\left\langle A_{0} \varphi, \varphi\right\rangle=\left\langle A_{0}^{\frac{1}{2}} \varphi, A_{0}^{\frac{1}{2}} \varphi\right\rangle=\|\varphi\|_{0}^{2}=\left\langle A_{0} \varphi, \varphi\right\rangle \leq \alpha_{0}\|\varphi\|^{2},
$$

for all $\varphi \in V$. Thus the two norms $\|\cdot\|$ and $\|\cdot\|_{0}$ on $V$ are equivalent.

The assumption of Hölder continuity on $g$ and the fact that $\left\{T_{0}(t ; \bar{q}): t \geq 0\right\}$, the semigroup of bounded linear operators on $H$ generated by the operator $-A_{0}$, is analytic, are sufficient to conclude that $A_{0} v(t) \in H$ for almost all $t>0$. It follows that $v(t) \in D_{0}$, a.e. $t>0$, and from (2.7), we obtain that

$$
\left\langle D_{t} v(t), A_{0} v(t)\right\rangle+\left|A_{0} v(t)\right|^{2}=\left\langle g(t), A_{0} v(t)\right\rangle, \quad \text { a.e. } t>0,
$$

and therefore that

$$
\frac{1}{2} D_{t}\|v(t)\|_{0}^{2}+\left|A_{0} v(t)\right|^{2} \leq|g(t)|\left|A_{0} v(t)\right| \leq \frac{1}{2}|g(t)|^{2}+\frac{1}{2}\left|A_{0} v(t)\right|^{2}, \quad \text { a.e. } t>0 .
$$

Integrating the above estimate from 0 to $t$, and recalling (2.8), we find that

$$
\left|v(t)\left\|_{0}^{2}+\int_{0}^{t}\left|A_{0} v(s)\right|^{2} d s \leq\right\| v_{0}\left\|_{0}^{2}+\int_{0}^{t}|g(s)|^{2} d s \leq\right\| v_{0}\left\|_{0}^{2}+\right\| g\right|_{L_{2}(0, \infty ; H)}^{2} \quad t \geq 0,
$$

from which the desired conclusion follows.

For each $t>0$, let $e(t)=u(t)-v(t)$. We would like to find a control input, $f$, such that

$$
\lim _{t \rightarrow \infty}|e(t)|=0
$$

with $f$ remaining, in some sense, bounded (for example, bounded energy; $f \in L_{2}\left(0, \infty ; V^{*}\right)$ ). If the plant (i.e. $\bar{q}$ ) were known, the convergence in $(2.16)$ could be achieved by setting

$$
f(t)=A(\bar{q}) u(t)-A_{0} u(t)+g(t), \quad \text { a.e. } t>0 .
$$

For then $e$ would satisfy

$$
\left\langle D_{t} e(t), \varphi\right\rangle+\left\langle A_{0} e(t), \varphi\right\rangle=0, \quad \varphi \in V, \text { a.e. } t>0,
$$




$$
\epsilon(0)=\epsilon_{0},
$$

where $\epsilon_{0}=u_{0}-v_{0} \in H$. It follows from Assumption (A4) and (2.1) that

$$
|e(t)| \leq e^{-\rho_{0} K^{-2} t}\left|\epsilon_{0}\right|, \quad t \geq 0,
$$

and consequently that $(2.16)$ is satisfied. The closed loop system is given by

$$
\begin{gathered}
\left\langle D_{t} u(t), \varphi\right\rangle+\left\langle A_{0} u(t), \varphi\right\rangle=\langle g(t), \varphi\rangle, \quad \varphi \in V, \text { a.є. } t>0, \\
u(0)=u_{0},
\end{gathered}
$$

Theorem 2.2 For the non-adaptive closed loop system given by (2.5), (2.6), (2.17), or equivalently, (2.18), (2.19), we have $f(t) \in \hat{V}^{*}$ for a.e. $t>0$, and the following results.

(i) If $g \in L_{2}\left(0, \infty ; V^{*}\right)$, then $u \in L_{\infty}(0, \infty ; H) \cap L_{2}(0, \infty ; V)$ and, moreover, $f \in L_{2}\left(0, \infty ; V^{*}\right)$.

(ii) If $g \in L_{\infty}(0, \infty ; V)$ and $u_{0} \in V$, then $u \in L_{\infty}(0, \infty ; V)$ and $f \in L_{\infty}\left(0, \infty ; V^{*}\right)$.

(iii) If the operator $A_{0}$ is symmetric in the sense of (2.13), $u_{0} \in V$, and $g \in L_{2}(0, \infty ; H)$ and satisfies (2.12), then $u(t) \in D_{0}$, a.e. $t>0, u \in L_{\infty}(0, \infty ; V)$ and $A_{0} u \in L_{2}(0, \infty ; H)$. If, in addition,

(a) $g \in L_{\infty}\left(0, \infty ; V^{*}\right)$, then $f \in L_{\infty}\left(0, \infty ; V^{*}\right)$,

or

(b) for $\varphi \in D_{0}, A(\bar{q}) \varphi \in H$ and

$$
|A(\bar{q}) \varphi| \leq \gamma\left|A_{0} \varphi\right|, \quad \varphi \in D_{0},
$$

for some $\gamma>0$, then $f \in L_{2}(0, \infty ; H)$.

Proof. The fact that $f(t) \in \hat{V}^{*}$, a.e. $t>0$, follows immediately from Assumption $(A 5)$ and the assumption that $g(t) \in \hat{V}^{*}$, a.e. $t>0$.

To establish $(i)$, as in the proof of Theorem 2.1, for almost every $t>0,(2.18)$ implies that

$$
\begin{aligned}
\frac{1}{2} D_{t}|u(t)|^{2} & =\left\langle-A_{0} u(t)+g(t), u(t)\right\rangle \\
& \leq-\rho_{0}\|u(t)\|^{2}+\|g(t)\|_{*} \mid u(t) \| \\
& \leq-\frac{\rho_{0}}{2}\|u(t)\|^{2}+\frac{1}{2}\|g(t)\|_{*}^{2},
\end{aligned}
$$

and hence that

$$
|u(t)|^{2}+\rho_{0} \int_{0}^{t}\left\|\left.u(s)\right|^{2} d s \leq\left|u_{0}\right|^{2}+\right\| g \|_{L_{2}\left(0, \infty ; V^{*}\right)}^{2}, \quad t>0 .
$$

Consequently, $u \in L_{\infty}(0, \infty ; H) \cap L_{2}(0, \infty ; V)$. Assumptions $(A 1)$ and $(A 3)$ and the definition of the control input $f$ given in (2.17) yield

$$
\|f(t)\|_{*} \leq\left\{\alpha_{1}|\bar{q}|_{Q}+\alpha_{2}+\alpha_{0}\right\}\|u(t)\|+\|\left. g(t)\right|_{*}, \quad \text { a.e. } t>0,
$$

from which it follows that $f \in L_{2}\left(0, \infty ; V^{*}\right)$. 
The result given in (ii) follows immediately from (2.10), the fact that (2.18) and (2.19) imply that

$$
u(t)=T_{0}(t) u_{0}+\int_{0}^{t} T_{0}(t-s) g(s) d s, \quad t \geq 0,
$$

and $(2.20)$.

To verify the claim in (iii), following the proof of Theorem 2.1, we first have that $u(t) \in D_{0}$, a.e. $t>0$. Then, (2.18) yields

$$
\left\langle D_{t} u(t), A_{0} u(t)\right\rangle+\left|A_{0} u(t)\right|^{2}=\left\langle g(t), A_{0} u(t)\right\rangle, \quad \text { a.e. } t>0,
$$

and therefore that

$$
\frac{1}{2} D_{t}|| u(t) \|_{0}^{2}+\left|A_{0} u(t)\right|^{2} \leq|g(t)|\left|A_{0} u(t)\right| \leq \frac{1}{2}|g(t)|^{2}+\frac{1}{2}\left|A_{0} u(t)\right|^{2}, \quad \text { a.e. } t>0 .
$$

Integrating (2.21) from 0 to $t$, and recalling (2.19) and (2.15), we find that

$$
\rho_{0}\|u(t)\|^{2}+\int_{0}^{t}\left|A_{0} u(s)\right|^{2} d s \leq \alpha_{0}\left\|u_{0}\right\|^{2}+\int_{0}^{t}|g(s)|^{2} d s \quad t \geq 0 .
$$

Clearly, the above estimate, (2.22), together with $g \in L_{2}(0, \infty ; H)$ imply that $u \in L_{\infty}(0, \infty ; V)$ and $A_{0} u \in L_{2}(0, \infty ; H)$. The result given in (iii) (a) then follows immediately from (2.20), while the estimate

$$
|f(t)| \leq\{\gamma+1\}\left|A_{0} u(t)\right|+|g(t)|, \quad \text { a.e. } t>0,
$$

yields the result given in $($ iii $)(b)$.

The importance of Theorem 2.2 lies in the fact that it serves as an upper bound for the results we can hope to obtain for a corresponding adaptive scheme wherein the plant, $\bar{q}$, is unknown and is estimated in real time.

Since $\bar{q}$ is in fact unknown, we set

$$
f(t)=A(q(t)) u(t)-A_{0} u(t)+g(t), \quad \text { a.e. } t>0,
$$

or

$$
\langle f(t), \varphi\rangle=\langle A(q(t)) u(t), \varphi\rangle-\left\langle A_{0} u(t), \varphi\right\rangle+\langle g(t), \varphi\rangle, \quad \varphi \in V, \text { a.e. } t>0,
$$

where for each $t>0, q(t) \in Q$ denotes an adaptively updated estimate for $\bar{q}$. Once again, $f(t) \in \hat{V}^{*}$, a.e. $t>0$ follows from Assumption $(A 5)$ and the fact that $g(t) \in \hat{V}^{*}$, a.e. $t>0$. By analogy to the finite dimensional case, and for the purpose of forcing an appropriate energy functional which will be defined in the next section when we consider convergence, we let the adaptation law for $q$ be given by

$$
\begin{gathered}
\left\langle D_{t} q(t), p\right\rangle_{Q}+\left\langle A_{1}(p) u(t), e(t)\right\rangle=0, \quad p \in Q, \text { a.e. } t>0, \\
q(0)=q_{0},
\end{gathered}
$$

where $q_{0} \in Q$, and $e(t)=u(t)-v(t), t>0$. The closed loop system is then given by

$$
\begin{gathered}
\left\langle D_{t} u(t), \varphi\right\rangle+\left\langle A_{0} u(t), \varphi\right\rangle+\left\langle A_{1}(\bar{q}-q(t)) u(t), \varphi\right\rangle=\langle g(t), \varphi\rangle, \quad \varphi \in V, \text { a.e. } t>0, \\
\left\langle D_{t} v(t), \varphi\right\rangle+\left\langle A_{0} v(t), \varphi\right\rangle=\langle g(t), \varphi\rangle, \quad \varphi \in V, \text { a.e. } t>0, \\
\left\langle D_{t} q(t), p\right\rangle_{Q}+\left\langle A_{1}(p) u(t), u(t)-v(t)\right\rangle=0, \quad p \in Q, \text { a.e. } t>0, \\
u(0)=u_{0}, \quad v(0)=v_{0}, \quad q(0)=q_{0} .
\end{gathered}
$$


We are interested in showing that the nonlinear system $(2.27)-(2.30)$ is, at least in some sense and under some set of minimally realizable assumptions, well posed. Recalling that $u(t)=$ $\epsilon(t)+v(t)$, and defining the parameter error, $r$, to be

$$
r(t)=q(t)-\bar{q}, \quad t>0,
$$

we consider instead the equivalent problem of establishing a well posedness result for the nonlinear system

$$
\begin{gathered}
\left\langle D_{t} e(t), \varphi\right\rangle+\left\langle A_{0} e(t), \varphi\right\rangle-\left\langle A_{1}(r(t))\{\epsilon(t)+v(t)\}, \varphi\right\rangle=0, \quad \varphi \in V, \text { a.e. } t>0, \\
\left\langle D_{t} v(t), \varphi\right\rangle+\left\langle A_{0} v(t), \varphi\right\rangle=\langle g(t), \varphi\rangle, \quad \varphi \in V, \text { a.e. } t>0, \\
\left\langle D_{t} r(t), p\right\rangle_{Q}+\left\langle A_{1}(p)\{e(t)+v(t)\}, e(t)\right\rangle=0, \quad p \in Q, \text { a.e. } t>0, \\
e(0)=\epsilon_{0}, \quad v(0)=v_{0}, \quad r(0)=r_{0},
\end{gathered}
$$

where $r_{0}=q_{0}-\bar{q} \in Q$. In the discussion to follow, we present two approaches to demonstrating the well-posedness of the closed-loop system, (2.32)-(2.35). We will first demonstrate the existence of a unique strong solution using the theory of semilinear equations with analytic semigroups. The second approach is based upon an application of an implicit function theorem. Necessarily, each of the two approaches will require its own set of additional hypotheses which must be satisfied in order for there to exist a unique solution. We note that the nonlinear system (2.32)-(2.35) is the one we will be using to establish the tracking error and parameter convergence in the next section. We note also that the skew-self-adjoint-like structure of the system (2.32)-(2.35) plays an essential role in the analysis to follow in Sections 2.1 and 2.2 .

\subsection{An Analytic Semigroup Approach to Closed-Loop Well-Posedness}

Let $X=H \times H \times Q$ be endowed with the inner product

$$
\left\langle\left(\varphi_{1}, \psi_{1}, q_{1}\right),\left(\varphi_{2}, \psi_{2}, q_{2}\right)\right\rangle_{X}=\left\langle\varphi_{1}, \varphi_{2}\right\rangle+\left\langle\psi_{1}, \psi_{2}\right\rangle+\left\langle q_{1}, q_{2}\right\rangle_{Q}, \quad\left(\varphi_{i}, \psi_{i}, q_{i}\right) \in X, i=1,2,
$$

and let $|\cdot|_{X}$ denote the corresponding induced norm. Thus $\left\{X,\langle\cdot, \cdot\rangle_{X},|\cdot|_{X}\right\}$ is a Hilbert space. Let $Y=V \times V \times Q$ be endowed with the norm

$$
\|\left.(\varphi, \psi, q)\right|_{Y}=\left(\|\varphi\|^{2}+\|\psi\|^{2}+|q|_{Q}^{2}\right)^{\frac{1}{2}}, \quad(\varphi, \psi, q) \in Y
$$

Then $\left\{Y,\|\cdot\|_{Y}\right\}$ is a reflexive Banach space which is densely and continuously embedded in $X$. It follows that

$$
Y \hookrightarrow X \hookrightarrow Y^{*}
$$

with the embeddings dense and continuous. For $\lambda>0$, define the linear operator $\mathcal{A}_{\lambda}: Y \rightarrow Y^{*}$ by

$$
\left\langle\mathcal{A}_{\lambda}(e, v, r),(\varphi, \psi, q)\right\rangle_{Y^{*}, Y}=\left\langle A_{0} e, \varphi\right\rangle+\left\langle A_{0} v, \psi\right\rangle+\langle\lambda r, q\rangle_{Q},
$$

for $(e, v, r),(\varphi, \psi, q) \in Y$. In the above definition, $\langle\cdot, \cdot\rangle_{Y, Y^{*}}$ denotes the duality pairing between $Y^{*}$ and $Y$ induced by the $X$-inner product via the dense and continuous embeddings given in (2.36). Recalling that $D_{0}=\operatorname{Dom}\left(A_{0}\right)=\left\{\varphi \in V: A_{0} \varphi \in H\right\}$, for $\lambda>0$, define the operator $A_{\lambda}: \operatorname{Dom}\left(A_{\lambda}\right) \subset X \rightarrow X$ by

$$
\operatorname{Dom}\left(A_{\lambda}\right)=\left\{(\varphi, \psi, q) \in Y: \mathcal{A}_{\lambda}(\varphi, \psi, q) \in X\right\}=D_{0} \times D_{0} \times Q,
$$




$$
A_{\lambda}(\varphi, \psi, q)=\mathcal{A}_{\lambda}(\varphi, \psi, q), \quad(\varphi, \psi, q) \in \operatorname{Dom}\left(A_{\lambda}\right)
$$

Note that $\operatorname{Dom}\left(A_{\lambda}\right)=\operatorname{Dom}(A)$ is independent of $\lambda>0$, that for $\lambda>0,-A_{\lambda}$ is the infinitesimal generator of a uniformly exponentially stable analytic semigroup, $\left\{T_{\lambda}(t): t \geq 0\right\}$, on $X, Y$, and $Y^{*}$, and that $0 \in \rho\left(-A_{\lambda}\right)$, the resolvent set of $-A_{\lambda}$.

For $\varphi \in V$, define the operator $B(\varphi): Q \rightarrow V^{*}$ by

$$
\langle B(\varphi) q, \psi\rangle=\left\langle A_{1}(q) \varphi, \psi\right\rangle \quad q \in Q, \psi \in V .
$$

Assumptions (A1) and (A2) imply that for $\varphi \in V, B(\varphi) \in \mathcal{L}\left(Q, V^{*}\right)$ with $\|B(\varphi)\| \leq \alpha_{1}\|\varphi\|$. Recalling that $V$ was assumed to be reflexive, and that $Q$ is a Hilbert space, for $\varphi \in V$, let $B(\varphi)^{\prime} \in \mathcal{L}(V, Q)$ denote the Banach space adjoint of $B(\varphi)$. That is, for $\varphi \in V$, we have

$$
\left\langle B(\varphi)^{\prime} \psi, q\right\rangle_{Q}=\langle B(\varphi) q, \psi\rangle=\left\langle A_{1}(q) \varphi, \psi\right\rangle, \quad \psi \in V, q \in Q .
$$

For $\lambda>0$, define $G_{\lambda}: \mathbf{R}^{+} \times Y \rightarrow Y^{*}$ by

$$
\left\langle G_{\lambda}(t, \Phi), \Psi\right\rangle_{Y^{*}, Y}=\langle B(e+v) r, \varphi\rangle+\langle g(t), \psi\rangle+\left\langle\lambda r-B(e+v)^{\prime} \epsilon, q\right\rangle_{Q},
$$

where $t \geq 0, \Phi=(e, v, r) \in Y$ and $\Psi=(\varphi, \psi, q) \in Y$.

We consider the system (2.32)-(2.35) written as

$$
\begin{gathered}
\left\langle D_{t} x(t), \Phi\right\rangle_{Y^{*}, Y}+\left\langle\mathcal{A}_{\lambda} x(t), \Phi\right\rangle_{Y^{*}, Y}=\left\langle G_{\lambda}(t, x(t)), \Phi\right\rangle_{Y^{*}, Y}, \quad \Phi \in Y, \text { a.e. } t>0, \\
x(0)=x_{0},
\end{gathered}
$$

where $\lambda>0$, and for each $t \geq 0, x(t)=(e(t), v(t), r(t))$. Under appropriate additional assumptions on the input reference signal, $g$, the initial data, $e_{0}, v_{0}$, and $r_{0}$, and the plant (i.e. the operator $A_{1}(q)$, for $q \in Q$ ) we establish the existence of a unique solution to the system (2.39),(2.40) by first establishing the existence of a unique local strong solution to the initial value problem in $X$ given by

$$
\begin{gathered}
D_{t} x(t)+A_{\lambda} x(t)=G_{\lambda}(t, x(t)), \quad \text { a.e. } t>0, \\
x(0)=x_{0},
\end{gathered}
$$

and then showing that it is possible to continue this solution for all $t>0$. By a strong (or classical) solution on the interval $[0, T)$ to the initial value problem $(2.41),(2.42)$ we mean a function $x:[0, T) \rightarrow X$ which is continuous on $[0, T)$, continuously differentiable on $(0, T)$, $x(t) \in \operatorname{Dom}(A)=\operatorname{Dom}\left(A_{\lambda}\right)$ for $t \in(0, T),(2.41)$ is satisfied for $t \in(0, T)$, and $(2.42)$ is satisfied.

To establish that the initial value problem $(2.41),(2.42)$ is well posed, we require the following additional assumptions.

(A6) ( $q$-Independent Domain) The subset of $V, D_{1}=\operatorname{Dom}\left(A_{1}(q)\right)$ is independent of $q \in Q$ and for some $\alpha \in(0,1), \operatorname{Dom}\left(A_{0}^{\alpha}\right) \subset D_{1}$.

(A7) ( $A_{0}^{\alpha}$-Boundedness) There exist $\beta_{1}>0$ such that for $\alpha$ as in Assumption (A6), we have

$$
\left|A_{1}(q) \varphi\right| \leq \beta_{1}|q|_{Q}\left|A_{0}^{\alpha} \varphi\right|, \quad q \in Q, \varphi \in \operatorname{Dom}\left(A_{0}^{\alpha}\right),
$$

(A8) $\left(A_{0}^{\alpha}\right.$-Lipschitz) There exist $\gamma_{1}>0$ such that for $\alpha$ as in Assumption (A6), we have

$$
\left|A_{1}(q) \varphi-A_{1}(q) \psi\right| \leq \gamma_{1}|q|_{Q}\left|A_{0}^{\alpha} \varphi-A_{0}^{\alpha} \psi\right|, \quad q \in Q, \varphi, \psi \in \operatorname{Dom}\left(A_{0}^{\alpha}\right),
$$


(A9) (Hölder Continuity) For $t \geq 0, g(t) \in H$, and there exists $\nu \in(0,1]$ and $\delta>0$ such that

$$
|g(t)-g(s)| \leq \delta|t-s|^{\nu}, \quad t, s \geq 0 .
$$

Note that Assumptions ( $A 3)$ and $(A 4)$ are sufficient for fractional powers of the operator $A_{0}$ to be well defined (see, for example, [32]).

Theorem 2.3 Suppose that Assumptions $(A 1)-(A 9)$ hold, and that $e_{0}, v_{0} \in \operatorname{Dom}\left(A_{0}^{\alpha}\right)$, where $\alpha \in(0,1)$ is as in Assumption (A6). Then there exists a $T=T\left(x_{0}\right)>0$ such that the initial value problem (2.41), (2.42) has a unique local solution $x \in C([0, T) ; X) \cap C^{1}((0, T) ; X)$.

Proof. For $\alpha \in(0,1)$, the linear operator $A_{0}^{\alpha}$ is closed and invertible with domain, $\operatorname{Dom}\left(A_{0}^{\alpha}\right)$, dense in $H$. For the $\alpha$ in Assumption $(A 6)$, let $H_{\alpha}$ denote the space $\operatorname{Dom}\left(A_{0}^{\alpha}\right)$ endowed with the graph norm, $\|\cdot\|_{\alpha}$, corresponding to $A_{0}^{\alpha}$. That is, for $\varphi \in \operatorname{Dom}\left(A_{0}^{\alpha}\right),\|\varphi\|_{\alpha}=|\varphi|+\left|A_{0}^{\alpha} \varphi\right|$. Note that since $A_{0}^{\alpha}$ is closed, $H_{\alpha}$ is a Banach space, and since $A_{0}^{\alpha}$ is invertible, the norm $\|\cdot\|_{\alpha}$ is equivalent to the norm $|\cdot|_{\alpha}$ on $\operatorname{Dom}\left(A_{0}^{\alpha}\right)$ given by $|\varphi|_{\alpha}=\left|A_{0}^{\alpha} \varphi\right|$, for $\varphi \in \operatorname{Dom}\left(A_{0}^{\alpha}\right)$. Define the Banach space $\left\{X_{\alpha},|\cdot| X_{\alpha}\right\}$ by $X_{\alpha}=H_{\alpha} \times H_{\alpha} \times Q$ with $|\Phi|_{X_{\alpha}}=\left|\varphi_{1}\right|_{\alpha}+\left|\varphi_{2}\right|_{\alpha}+\left|\varphi_{3}\right|_{Q}$, for $\Phi=\left(\varphi_{1}, \varphi_{2}, \varphi_{3}\right) \in X_{\alpha}$.

The theorem will follow at once from Theorem 6.3.1 in [32] once we have established that for some $\lambda>0$ and any neighborhood, $U \subset X_{\alpha}$, of $x_{0}, U=\left\{x \in X_{\alpha}:\left|x-x_{0}\right|_{X_{\alpha}}<\varepsilon\right\}$, there exists a constant $L=L(U, \lambda)=L\left(\varepsilon, x_{0}, \lambda\right)>0$, such that

$$
\begin{aligned}
& \left|G_{\lambda}(t, \Phi)-G_{\lambda}(s, \Psi)\right|_{X} \\
& \quad \leq L\left\{|t-s|^{\nu}+\left|A_{0}^{\alpha} \varphi_{1}-A_{0}^{\alpha} \psi_{1}\right|+\left|A_{0}^{\alpha} \varphi_{2}-A_{0}^{\alpha} \psi_{2}\right|+\left|\varphi_{3}-\psi_{3}\right|_{Q}\right\}, \\
& \quad=L\left\{|t-s|^{\nu}+\left|\varphi_{1}-\psi_{1}\right|_{\alpha}+\left|\varphi_{2}-\psi_{2}\right|_{\alpha}+\left|\varphi_{3}-\psi_{3}\right|_{Q}\right\}, \quad t, s>0,
\end{aligned}
$$

for all $\Phi=\left(\varphi_{1}, \varphi_{2}, \varphi_{3}\right), \Psi=\left(\psi_{1}, \psi_{2}, \psi_{3}\right) \in U$. Let $\lambda>0$ and $\Phi=\left(\varphi_{1}, \varphi_{2}, \varphi_{3}\right), \Psi=\left(\psi_{1}, \psi_{2}, \psi_{3}\right) \in$ $U$, and consider for $t, s>0$,

$$
\begin{aligned}
& \left|G_{\lambda}(t, \Phi)-G_{\lambda}(s, \Psi)\right|_{X}^{2}=\left|B\left(\varphi_{1}+\varphi_{2}\right) \varphi_{3}-B\left(\psi_{1}+\psi_{2}\right) \psi_{3}\right|^{2}+ \\
& \quad|g(t)-g(s)|^{2}+\left|\lambda\left\{\varphi_{3}-\psi_{3}\right\}-\left\{B\left(\varphi_{1}+\varphi_{2}\right)^{\prime} \varphi_{1}-B\left(\psi_{1}+\psi_{2}\right)^{\prime} \psi_{1}\right\}\right|_{Q}^{2} .
\end{aligned}
$$

Now, Assumptions ( $A 7)$ and (A8) imply that

$$
\begin{aligned}
& \left|B\left(\varphi_{1}+\varphi_{2}\right) \varphi_{3}-B\left(\psi_{1}+\psi_{2}\right) \psi_{3}\right| \\
& \quad \leq\left|B\left(\varphi_{1}+\varphi_{2}\right) \varphi_{3}-B\left(\varphi_{1}+\varphi_{2}\right) \psi_{3}\right|+\left|B\left(\varphi_{1}+\varphi_{2}\right) \psi_{3}-B\left(\psi_{1}+\psi_{2}\right) \psi_{3}\right| \\
& \quad \leq \beta_{1}\left|\varphi_{3}-\psi_{3}\right|_{Q}\left\{\left|A_{0}^{\alpha} \varphi_{1}\right|+\left|A_{0}^{\alpha} \varphi_{2}\right|\right\}+\gamma_{1}\left|\psi_{3}\right|_{Q}\left\{\left|A_{0}^{\alpha} \varphi_{1}-A_{0}^{\alpha} \psi_{1}\right|+\left|A_{0}^{\alpha} \varphi_{2}-A_{0}^{\alpha} \psi_{2}\right|\right\} \\
& \quad=\beta_{1}\left|\varphi_{3}-\psi_{3}\right|_{Q}\left\{\left|\varphi_{1}\right|_{\alpha}+\left|\varphi_{2}\right|_{\alpha}\right\}+\gamma_{1}\left|\psi_{3}\right|_{Q}\left\{\left|\varphi_{1}-\psi_{1}\right|_{\alpha}+\left|\varphi_{2}-\psi_{2}\right|_{\alpha}\right\} .
\end{aligned}
$$

Assumption (A9) implies that

$$
|g(t)-g(s)| \leq \delta|t-s|^{\nu}, \quad t, s \geq 0,
$$

for some $\nu>0$. Finally, using Assumptions ( $A 7)$ and ( $A 8)$, we obtain

$$
\begin{aligned}
& \left|\lambda\left\{\varphi_{3}-\psi_{3}\right\}-\left\{B\left(\varphi_{1}+\varphi_{2}\right)^{\prime} \varphi_{1}-B\left(\psi_{1}+\psi_{2}\right)^{\prime} \psi_{1}\right\}\right|_{Q} \\
& \quad \leq \lambda\left|\varphi_{3}-\psi_{3}\right|_{Q}+\sup _{|q|_{Q} \leq 1}\left|\left\langle\left\{B\left(\varphi_{1}+\varphi_{2}\right)^{\prime} \varphi_{1}-B\left(\psi_{1}+\psi_{2}\right)^{\prime} \psi_{1}\right\}, q\right\rangle_{Q}\right|
\end{aligned}
$$




$$
\begin{aligned}
& =\lambda\left|\varphi_{3}-\psi_{3}\right|_{Q}+\sup _{|q|_{Q} \leq 1}\left|\left\langle A_{1}(q)\left\{\varphi_{1}+\varphi_{2}\right\}, \varphi_{1}\right\rangle-\left\langle A_{1}(q)\left\{\psi_{1}+\psi_{2}\right\}, \psi_{1}\right\rangle\right| \\
& \leq \lambda\left|\varphi_{3}-\psi_{3}\right|_{Q}+\sup _{|q|_{Q} \leq 1}\left|\left\langle A_{1}(q)\left\{\varphi_{1}+\varphi_{2}\right\}-A_{1}(q)\left\{\psi_{1}+\psi_{2}\right\}, \varphi_{1}\right\rangle\right| \\
& +\sup _{|q|_{\varepsilon} \leq 1}\left|\left\langle A_{1}(q)\left\{\psi_{1}+\psi_{2}\right\}, \varphi_{1}-\psi_{1}\right\rangle\right| \\
& \leq \lambda\left|\varphi_{3}-\psi_{3}\right|_{Q}+\sup _{\mid}\left|A_{1}(q)\left\{\varphi_{1}+\varphi_{2}\right\}-A_{1}(q)\left\{\psi_{1}+\psi_{2}\right\}\right|\left|\varphi_{1}\right| \\
& |q|_{Q} \leq 1 \\
& +\sup \left|A_{1}(q)\left\{\psi_{1}+\psi_{2}\right\}\right|\left|\varphi_{1}-\psi_{1}\right| \\
& |q|_{Q} \leq 1 \\
& \leq \lambda\left|\varphi_{3}-\psi_{3}\right|_{Q}+\gamma_{1}\left|\varphi_{1}\right|\left\{\left|A_{0}^{\alpha} \varphi_{1}-A_{0}^{\alpha} \psi_{1}\right|+\left|A_{0}^{\alpha} \varphi_{2}-A_{0}^{\alpha} \psi_{2}\right|\right\} \\
& +\beta_{1}\left\{\left|A_{0}^{\alpha} \psi_{1}\right|+\left|A_{0}^{\alpha} \psi_{2}\right|\right\}\left|\varphi_{1}-\psi_{1}\right| \\
& \leq \lambda\left|\varphi_{3}-\psi_{3}\right|_{Q}+\gamma_{1} \kappa_{\alpha}\left|\varphi_{1}\right|_{\alpha}\left\{\left|\varphi_{1}-\psi_{1}\right|_{\alpha}+\left|\varphi_{2}-\psi_{2}\right|_{\alpha}\right\} \\
& +\beta_{1} \kappa_{\alpha}\left\{\left|\psi_{1}\right|_{\alpha}+\left|\psi_{2}\right|_{\alpha}\right\}\left|\varphi_{1}-\psi_{1}\right|_{\alpha},
\end{aligned}
$$

where $\kappa_{\alpha}$ is such that $|\varphi| \leq\|\varphi\|_{\alpha} \leq \kappa_{\alpha}|\varphi|_{\alpha}$, for $\varphi \in H_{\alpha}$. Combining (2.47)-(2.50), we obtain (2.46), and the theorem is proved.

In order to extend the local solution guaranteed to exist in Theorem 2.3 we require the estimate given in the following lemma.

Lemma 2.4 Let $x=(e, v, r)$ be the unique solution to the initial value problem $(2.41),(2.42)$ guaranteed to exist on the interval $[0, T)$ by Theorem 2.3. It then follows that

$$
|x(t)|_{X}^{2}+\rho_{0} \int_{0}^{t}\left\{\left\|\left.e(s)\right|^{2}+\right\| v(s) \|^{2}\right\} d s \leq\left|x_{0}\right|_{X}^{2}+\frac{1}{\rho_{0}} \int_{0}^{t}\|g(s)\|_{*}^{2} d s, \quad 0 \leq t<T .
$$

Proof. For $s \in[0, T)$, using (2.41), we obtain

$$
\begin{aligned}
\frac{1}{2} D_{t}|x(s)|_{X}^{2} & =\left\langle D_{t} x(s), x(s)\right\rangle_{X} \\
& =-\left\langle A_{\lambda} x(s), x(s)\right\rangle_{X}+\left\langle G_{\lambda}(s, x(s)), x(s)\right\rangle_{X} \\
& =-\left\langle A_{0} e(s), e(s)\right\rangle-\left\langle A_{0} v(s), v(s)\right\rangle+\langle g(s), v(s)\rangle \\
& \leq-\rho_{0}\|e(s)\|^{2}-\rho_{0}\|v(s)\|^{2}+\|g(s)\|\left\|_{*}\right\| v(s) \| \\
& \leq-\frac{\rho_{0}}{2}\left\{\|e(s)\|^{2}+\|v(s)\|^{2}\right\}+\frac{1}{2 \rho_{0}}\|g(s)\|_{*}^{2} .
\end{aligned}
$$

Integrating both sides of (2.52) from 0 to $t$, and using (2.42), we obtain (2.51), and the lemma is proved.

Note that the proof of Lemma 2.4 given above does not explicitly require that the additional assumptions $(A 6)-(A 9)$ be satisfied.

Theorem 2.5 Suppose that Assumptions (A1)-(A9) hold, and that $\epsilon_{0}, v_{0} \in \operatorname{Dom}\left(A_{0}^{\alpha}\right)$, where $\alpha \in(0,1)$ is as in Assumption (A6). Then the initial value problem (2.41),(2.42) has a unique solution, $x=(e, v, r)$, which exists for all $t \geq 0$.

Proof. The local solution, $x$, to the initial value problem (2.41),(2.42) guaranteed to exist by Theorem 2.3 can be continued so long as $|x(t)|_{X_{\alpha}}$ remains bounded. We show that this is in fact the case by using Lemma 2.4 to argue that $|x(t)|_{X_{\alpha}}$ remains bounded as $t \uparrow T$. 
For $t \in[0, T)$ we have that

$$
x(t)=T_{\lambda}(t) x_{0}+\int_{0}^{t} T_{\lambda}(t-s) G_{\lambda}(s, x(s)) d s,
$$

and therefore that

$$
A_{\lambda}^{\alpha} x(t)=A_{\lambda}^{\alpha} T_{\lambda}(t) x_{0}+\int_{0}^{t} A_{\lambda}^{\alpha} T_{\lambda}(t-s) G_{\lambda}(s, x(s)) d s .
$$

Equivalently, we have

$$
\begin{gathered}
A_{0}^{\alpha} e(t)=A_{0}^{\alpha} T_{0}(t) e_{0}+\int_{0}^{t} A_{0}^{\alpha} T_{0}(t-s) B(e(s)+v(s)) r(s) d s, \\
A_{0}^{\alpha} v(t)=A_{0}^{\alpha} T_{0}(t) v_{0}+\int_{0}^{t} A_{0}^{\alpha} T_{0}(t-s) g(s) d s
\end{gathered}
$$

and

$$
\lambda^{\alpha} r(t)=\lambda^{\alpha} e^{-\lambda t} r_{0}+\int_{0}^{t} \lambda^{\alpha} e^{-\lambda(t-s)}\left\{\lambda r(s)-B(\epsilon(s)+v(s))^{\prime} \epsilon(s)\right\} d s .
$$

It follows from Assumptions ( $A 7)$ and (A9), (2.9), and Theorem 2.6.13 in [32] that

$$
\begin{aligned}
|e(t)|_{\alpha} & \leq e^{-\rho_{0} K^{-2} t}\left|\epsilon_{0}\right|_{\alpha}+\int_{0}^{t} M_{\alpha}(t-s)^{-\alpha} e^{-\rho_{0} K^{-2}(t-s)} \beta_{1}|r(s)|_{Q}\left\{|\epsilon(s)|_{\alpha}+|v(s)|_{\alpha}\right\} d s \\
& \leq\left|e_{0}\right|_{\alpha}+M_{\alpha} \beta_{1} \int_{0}^{t}(t-s)^{-\alpha}|r(s)|_{Q}|x(s)|_{X_{\alpha}} d s \\
|v(t)|_{\alpha} & \leq e^{-\rho_{0} K^{-2} t}\left|v_{0}\right|_{\alpha}+\int_{0}^{t} M_{\alpha}(t-s)^{-\alpha} e^{-\rho_{0} K^{-2}(t-s)}|g(s)| d s \\
& \leq e^{-\rho_{0} K^{-2} t}\left|v_{0}\right|_{\alpha}+\int_{0}^{t} M_{\alpha}(t-s)^{-\alpha} e^{-\rho_{0} K^{-2}(t-s)}\{|g(0)|+|g(s)-g(0)|\} d s \\
& \leq\left|v_{0}\right|_{\alpha}+M_{\alpha} \int_{0}^{t}(t-s)^{-\alpha}\left\{|g(0)|+\delta s^{\nu} d s\right. \\
& \leq\left|v_{0}\right|_{\alpha}+M_{\alpha}\left\{|g(0)|+\delta T^{\nu}\right\} \frac{T^{1-\alpha}}{1-\alpha}
\end{aligned}
$$

and

$$
\begin{aligned}
|r(t)|_{Q} & \leq\left|r_{0}\right|_{Q}+\int_{0}^{t} e^{-\lambda(t-s)}\left\{\lambda|r(s)|_{Q}+\left|B(e(s)+v(s))^{\prime} e(s)\right|_{Q}\right\} d s \\
& \leq\left|r_{0}\right|_{Q}+\int_{0}^{t}\left\{\lambda|r(s)|_{Q}+\sup _{|q|_{Q} \leq 1}\left|\left\langle A_{1}(q)\{e(s)+v(s)\}, e(s)\right\rangle\right|\right\} d s \\
& \leq\left|r_{0}\right|_{Q}+\int_{0}^{t}\left\{\lambda|r(s)|_{Q}+\beta_{1}|e(s)|\left\{\left|A_{0}^{\alpha} e(s)\right|+\left|A_{0}^{\alpha} v(s)\right|\right\} d s\right. \\
& \leq\left|r_{0}\right|_{Q}+\int_{0}^{t}\left\{\lambda|r(s)|_{Q}+\beta_{1}|e(s)|\left\{|e(s)|_{\alpha}+|v(s)|_{\alpha}\right\} d s\right. \\
& \leq\left|r_{0}\right|_{Q}+T^{\alpha} \int_{0}^{t} \max \left\{\lambda, \beta_{1}|e(s)|\right\}(t-s)^{-\alpha}|x(s)|_{X_{\alpha}} d s
\end{aligned}
$$


where $M_{\alpha}$ is a positive constant. Now Lemma 2.4 implies that for $s \in[0, T),|x(s)|_{X}$ is bounded. It follows that $s \in[0, T),|e(s)|$ and $|r(s)|_{Q}$ are bounded. Combining (2.53), (2.54), and (2.55), we obtain

$$
|x(t)|_{X_{\alpha}} \leq\left|x_{0}\right|_{X_{\alpha}}+M_{\alpha}\left\{|g(0)|+\delta T^{\nu}\right\} \frac{T^{1-\alpha}}{1-\alpha}+C \int_{0}^{t}(t-s)^{-\alpha}|x(s)|_{X_{\alpha}} d s,
$$

where $C>0$. It follows from Theorem 5.6.7 in [32] that $|x(t)|_{X_{\alpha}} \leq C_{1}$ on $[0, T)$ for some $C_{1}>0$, and the theorem is proved.

Theorem 2.5 yields the following regularity result for the controller $f$. We state it as a Corollary.

Corollary 2.6 Suppose that Assumptions (A1)-(A9) hold, and that $e_{0}, v_{0} \in \operatorname{Dom}\left(A_{0}^{\alpha}\right)$, where $\alpha \in(0,1)$ is as in Assumption $(A 6)$. If the operator $A_{2}$ is such that $D_{2}=\operatorname{Dom}\left(A_{2}\right)=\{\varphi \in$ $\left.V: A_{2} \varphi \in H\right\} \supset \operatorname{Dom}\left(A_{0}^{\alpha}\right)$, where $\alpha \in(0,1)$ is as in Assumption $(A 6)$, and satisfies a Lipschitz condition of the form

$$
\left|A_{2} \varphi-A_{2} \psi\right| \leq \gamma_{2}|\varphi-\psi|_{\alpha}, \quad, \varphi, \psi \in \operatorname{Dom}\left(A_{0}^{\alpha}\right),
$$

then the control law given in (2.23) or (2.24) satisfies $f(t) \in H, t>0$, and $f \in C((0, \infty) ; H)$.

Proof. For $t>0$, the controller, $f$, satisfies

$$
f(t)=A(q(t)) u(t)-A_{0} u(t)+g(t)=D_{t} u(t)+A(\bar{q}) u(t) .
$$

Theorem 2.5 implies that $u(t) \in \operatorname{Dom}\left(A_{0}\right), t>0$. It follows, therefore, that $u(t) \in \operatorname{Dom}\left(A_{0}^{\alpha}\right)$, $t>0$, and therefore that $u(t) \in D_{j}, j=1,2, t>0$. Consequently, $A(\bar{q}) u(t) \in H, t>0$, and hence, $f(t) \in H, t>0$. Theorem 2.5 also implies that $D_{t} u \in C((0, \infty) ; H)$, and for $s, t>0$, Assumption (A8) together with (2.57) imply that

$$
\begin{aligned}
|A(\bar{q}) u(t)-A(\bar{q}) u(s)| & \leq\left|A_{1}(\bar{q}) u(t)-A_{1}(\bar{q}) u(s)\right|+\left|A_{2} u(t)-A_{2} u(s)\right| \\
& \leq\left\{\gamma_{1}|\bar{q}|_{Q}+\gamma_{2}\right\}|u(t)-u(s)|_{\alpha} .
\end{aligned}
$$

Inspection of the proof of Theorem 6.3.1 in [32] immediately reveals that $u$ is continuous in $H_{\alpha}$. It follows from $(2.58)$ and $(2.59)$ that $f \in C((0, \infty) ; H)$, which establishes the corollary. $\square$

We provide a simple example which satisfies Assumptions $(A 1)-(A 9)$. Let $H=L_{2}(0,1)$, and let it be endowed with the standard inner product, $\langle\cdot, \cdot\rangle$, and corresponding induced norm, $|\cdot|$. Let $V=H_{L}^{1}(0,1)=\left\{\varphi \in H^{1}(0,1): \varphi(0)=0\right\}$, and let it be endowed with the norm $\| \cdot \mid$ given by

$$
\|\varphi\|=\left\{\int_{0}^{1}|D \varphi(x)|^{2} d x\right\}^{\frac{1}{2}}, \quad \varphi \in H_{L}^{1}(0,1) .
$$

Then $\{V,\|\cdot\|\}$ is a reflexive Banach space, and in fact, a Hilbert space, which is densely and continuously embedded in $H$. We have $|\varphi| \leq\|\varphi\|, \varphi \in H_{L}^{1}(0,1)$. Let $\hat{V}^{*}=V^{*}$, and let $Q=\mathbf{R}^{1}$ with $|q|_{Q}=|q|$, for $q \in \mathbf{R}$. We are interested in controlling the first order plant given by

$$
\frac{\partial u}{\partial t}(t, x)+\bar{q} \frac{\partial u}{\partial x}(t, x)=f(t, x), \quad 0<x<1, t>0,
$$

together with the boundary condition

$$
u(t, 0)=0, \quad t>0,
$$


and initial condition

$$
u(0, x)=u_{0}(x), \quad 0 \leq x \leq 1,
$$

where $\bar{q}>0, u_{0} \in L_{2}(0,1)$, and $t \rightarrow f(t, \cdot) \in L_{2}(0, T ; H)$, for each $T>0$.

For each $q \in \mathbf{R}^{1}$, let the operator $A_{1}(q): \operatorname{Dom}\left(A_{1}(q)\right) \subset H \rightarrow H$ be given by

$$
A_{1}(q) \varphi=q D \varphi, \quad \varphi \in D_{1},
$$

where $D_{1}=\operatorname{Dom}\left(A_{1}(q)\right)=V$. For each $q \in Q$, let $A(q)=A_{1}(q)$. It follows that $A_{2}$ is the zero operator, that $D_{1}$ is independent of $q \in Q$, and that $A_{1}(q): V \rightarrow V^{*}$. Moreover, for $q \in Q$, and $\varphi, \psi \in V$, we have that

$$
\left|\left\langle A_{1}(q) \varphi, \psi\right\rangle\right|=|q|_{Q}\left|\int_{0}^{1} D \varphi(x) \psi(x) d x\right| \leq|q|_{Q}|D \varphi||\psi|=|q|_{Q}|| \varphi|||\psi| \leq|q|_{Q}|| \varphi|||\psi| \mid .
$$

Consequently Assumption ( $A 1)$ is satisfied with $\alpha_{1}=1$. Assumption ( $\left.A 2\right)$ is trivially satisfied.

It is not difficult to show that the Hilbert space adjoint of the operator $A_{1}(\bar{q})$ is given by

$$
A_{1}(\bar{q})^{*} \varphi=-\bar{q} D \varphi, \quad \varphi \in D_{1}^{*},
$$

where $D_{1}^{*}=\operatorname{Dom}\left(A_{1}(\bar{q})^{*}\right)=H_{R}^{1}(0,1)=\left\{\varphi \in H^{1}(0,1): \varphi(1)=0\right\}$. For $\varphi \in H_{L}^{1}(0,1)$, we have that

$$
\left\langle A_{1}(\bar{q}) \varphi, \varphi\right\rangle=\bar{q} \int_{0}^{1} D \varphi(x) \varphi(x) d x=\frac{\bar{q}}{2} \int_{0}^{1} D \varphi(x)^{2} d x=\frac{\bar{q}}{2} \varphi(1)^{2} \geq 0,
$$

and for $\varphi \in H_{R}^{1}(0,1)$, that

$$
\left\langle A_{1}(\bar{q})^{*} \varphi, \varphi\right\rangle=-\bar{q} \int_{0}^{1} D \varphi(x) \varphi(x) d x=-\frac{\bar{q}}{2} \int_{0}^{1} D \varphi(x)^{2} d x=\frac{\bar{q}}{2} \varphi(0)^{2} \geq 0 .
$$

It follows that (see, for example, [25], Theorem I.4.5) the operator $-A_{1}(\bar{q})$ is maximal dissipative and therefore that it is the infinitesimal generator of a $\mathcal{C}_{0}$-semigroup of bounded linear operators (in fact, contractions), $\{S(t, \bar{q}): t \geq 0\}$, on $H=L_{2}(0,1)$. For each $t \geq 0$, the unique mild solution, $u(t)=u(t, \cdot)$ to the system $(2.59)-(2.61)$ is given by

$$
u(t)=S(t ; \bar{q}) u_{0}+\int_{0}^{t} S(t-s ; \bar{q}) f(s) d s,
$$

where for each $t \geq 0, f(t)=f(t, \cdot) \in L_{2}(0,1)$. When $u_{0} \in H_{L}^{1}(0,1)$, and $f$ is strongly continuously differentiable for $t \geq 0$, the function $u$ given by (2.63) is a strong solution. Such a solution certainly satisfies our minimal well-posedness requirement on the plant.

For the reference model, we consider the one dimensional heat equation given by

$$
\frac{\partial v}{\partial t}(t, x)-a_{0} \frac{\partial^{2} v}{\partial x^{2}}(t, x)=g(t, x) \quad 0<x<1, t>0,
$$

together with the boundary conditions

$$
v(t, 0)=0, \quad \text { and } \quad \frac{\partial v}{\partial x}(t, 1)=0, \quad t>0,
$$

and the initial conditions

$$
v(0, x)=v_{0}(x), \quad 0 \leq x \leq 1,
$$


where $a_{0}>0, v_{0} \in L_{2}(0,1)$, and $t \rightarrow g(t, \cdot) \in L_{2}\left(0, T ; V^{*}\right)$, for each $T>0$. In this case we have $A_{0} \in \mathcal{L}\left(V, V^{*}\right)$ given by

$$
\left\langle A_{0} \varphi, \psi\right\rangle=a_{0} \int_{0}^{1} D \varphi(x) D \psi(x) d x, \quad \varphi, \psi \in H_{L}^{1}(0,1) .
$$

It is immediately clear that Assumptions $(A 3)$ and $(A 4)$ are satisfied with $\alpha_{0}=\rho_{0}=a_{0}$. Moreover, we have $D_{0}=\operatorname{Dom}\left(A_{0}\right)=\left\{\varphi \in H_{L}^{1}(0,1): \varphi \in H^{2}(0,1), D \varphi(1)=0\right\}$, and that $A_{0}$ as an operator from $V$ into $V^{*}$ is symmetric, or as an operator on $H$ is self-adjoint. Assumption $(A 5)$ is trivially satisfied with the choice of $\hat{V}^{*}=V^{*}$.

Since $A_{0}$ is symmetric, $V=\operatorname{Dom}\left(A_{0}^{\frac{1}{2}}\right)$ (see [38]). Consequently, we have $\operatorname{Dom}\left(A_{0}^{\frac{1}{2}}\right)=V=$ $\operatorname{Dom}\left(A_{1}(q)\right)=D_{1}$. It follows that Assumption $(A 6)$ is satisfied with $\alpha=\frac{1}{2}$. Moreover, for $\varphi \in H_{L}^{1}(0,1)=V$, we have that

$$
\begin{aligned}
\left|A_{1}(q) \varphi\right|^{2} & =|q D \varphi|^{2}=|q|_{Q}^{2}|D \varphi|^{2}=|q|_{Q}^{2}\|\varphi\|^{2} \\
& =|q|_{Q}^{2} \frac{1}{a_{0}}\left\langle A_{0} \varphi, \varphi\right\rangle=\frac{1}{a_{0}}|q|_{Q}^{2}\left\langle A_{0}^{\frac{1}{2}} \varphi, A_{0}^{\frac{1}{2}} \varphi\right\rangle=\frac{1}{a_{0}}|q|_{Q}^{2}\left|A_{0}^{\frac{1}{2}}\right|^{2} .
\end{aligned}
$$

It follows that Assumptions $(A 7)$ and $(A 8)$ are satisfied with $\beta_{1}=\gamma_{1}=\frac{1}{\sqrt{a_{0}}}$. Thus, if $u_{0}, v_{0} \in$ $H_{L}^{1}(0,1)$, and $g$ is sufficiently regular (i.e. Assumption $(A 9)$ being satisfied), then the resulting closed-loop system will be well-posed.

\subsection{Closed-Loop Well-Posedness via an Implicit Function Theorem}

Assumptions $(A 6)-(A 9)$ can be rather restrictive and may preclude the consideration of certain classes of problems of interest. In particular, Assumption $(A 7)$ does not include the class of problems in which the plant and reference model dynamics are of the same order (i.e. $\alpha \in(0,1)$ ). Thus, for example the above theory does not allow for both a plant and reference model described by a diffusion (or heat) equation. To remedy this, we propose a somewhat different approach to demonstrating the well-posedness of the closed loop system, (2.27)-(2.7). Our argument is based upon an application of the Implicit Function Theorem (see, for example, [9]). Of course this approach requires additional assumptions as well. Indeed, in this case, we can only guarantee well-posedness for initial data which is sufficiently small in norm. That is, the plant must initially be close to the reference model, and we require a reasonably good initial guess for the unknown parameters. Also, to simplify the presentation, we make the following assumption on the linearity of the plant.

(A10) (Linearity of the Plant) For each $q \in Q, A_{1}(q): V \rightarrow V^{*}$ is linear.

Note that Assumptions $(A 1)$ and $(A 10)$ together imply that $A_{1}(q) \in \mathcal{L}\left(V, V^{*}\right)$, for each $q \in$ $Q$. We note that Assumption $(A 10)$ can be weakened quite a bit to allow for certain classes of nonlinear plants. However, the required technical assumptions would only complicate the exposition without significantly affecting its substance. Consequently, we opt for clarity, and leave the generalization to the reader.

We also require the following regularity assumption on the state, $v$, of the reference model, $(2.7),(2.8)$.

(A11) (Regularity of the Reference Model) The solution $v$ to the system (2.7), (2.8) satisfies

$$
v \in L_{2}(0, \infty ; V) \text {. }
$$


Theorem 2.1 (ii) provides sufficient conditions for Assumption (A11) to be satisfied.

We consider $v$ to be an exogenous signal, and consider the initial value problem given by

$$
\begin{gathered}
\left\langle D_{t} e(t), \varphi\right\rangle+\left\langle A_{0} e(t), \varphi\right\rangle-\left\langle A_{1}(r(t))\{e(t)+v(t)\}, \varphi\right\rangle=0, \quad \varphi \in V, \text { a.e. } t>0, \\
\left\langle D_{t} r(t), p\right\rangle_{Q}+\left\langle A_{1}(p)\{e(t)+v(t)\}, e(t)\right\rangle=0, \quad p \in Q, \text { a.e. } t>0, \\
\epsilon(0)=e_{0}, \quad r(0)=r_{0},
\end{gathered}
$$

Theorem 2.7 Suppose that Assumptions (A1)-(A5) and Assumptions (A10) and (A11) hold. Suppose further that $e_{0} \in V$. Then there exists a constant $C>0$ such that if

$$
\left\|e_{0}\right\|+\left|r_{0}\right|_{Q}<C
$$

then the initial value problem (2.64)-(2.66) has a unique solution $(e, r)$ with $e \in L_{2}(0, \infty ; V) \cap$ $H^{1}\left(0, \infty ; V^{*}\right)$ and $r \in L_{\infty}(0, \infty ; Q) \cap W^{1,1}(0, \infty ; Q)$.

Proof. The proof follows from an application of the Implicit Function Theorem (see, for example, [9]). We begin with the definition of the following Banach spaces. Let

$$
X=V \times Q
$$

with norm

$$
\|(\varphi, q)\|_{X}=\|\varphi\|+|q|_{Q}, \quad \varphi \in V, q \in Q
$$

let

$$
Y=\left\{L_{2}(0, \infty ; V) \cap H_{L}^{1}\left(0, \infty ; V^{*}\right)\right\} \times\left\{L_{\infty}(0, \infty ; Q) \cap W_{L}^{1,1}(0, \infty ; Q)\right\}
$$

with norm

$$
\|(\varphi, q)\|_{Y}=\left\{\int_{0}^{\infty}\|\varphi(t)\|^{2} d t\right\}^{\frac{1}{2}}+\left\{\int_{0}^{\infty}\left\|D_{t} \varphi(t)\right\|_{*}^{2} d t\right\}^{\frac{1}{2}}+e s s \sup _{t \in \mathbf{R}^{+}}|q(t)|_{Q}+\int_{0}^{\infty}\left|D_{t} q(t)\right|_{Q} d t
$$

for $\varphi \in L_{2}(0, \infty ; V) \cap H_{L}^{1}\left(0, \infty ; V^{*}\right)$ and $q \in L_{\infty}(0, \infty ; Q) \cap W_{L}^{1,1}(0, \infty ; Q)$, and let

$$
Z=L_{2}\left(0, \infty ; V^{*}\right) \times L_{1}(0, \infty ; Q),
$$

with norm

$$
\|(\varphi, q)\|_{Z}=\left\{\int_{0}^{\infty}\|\varphi(t)\|_{*}^{2} d t\right\}^{\frac{1}{2}}+\int_{0}^{\infty}|q(t)|_{Q} d t
$$

for $\varphi \in L_{2}\left(0, \infty ; V^{*}\right)$ and $q \in L_{1}(0, \infty ; Q)$. The subscript $L$ in the above spaces denotes homogeneous boundary conditions at the left endpoint of the interval.

Define the function $\mathcal{F}: X \times Y \rightarrow Z$ by

$$
\mathcal{F}(x, y)=\left(\mathcal{F}_{1}(x, y), \mathcal{F}_{2}(x, y)\right), \quad x=\left(x_{1}, x_{2}\right) \in X, y=\left(y_{1}, y_{2}\right) \in Y,
$$

where $\mathcal{F}_{1}: X \times Y \rightarrow L_{2}\left(0, \infty ; V^{*}\right)$ is given by

$\mathcal{F}_{1}(x, y)=D_{t} y_{1}+A_{0}\left\{y_{1}+x_{1}\right\}-B\left(y_{1}+x_{1}+v\right)\left\{y_{2}+x_{2}\right\}, \quad x=\left(x_{1}, x_{2}\right) \in X, y=\left(y_{1}, y_{2}\right) \in Y$, and $\mathcal{F}_{2}: X \times Y \rightarrow L_{1}(0, \infty ; Q)$ is given by

$$
\mathcal{F}_{2}(x, y)=D_{t} y_{2}+B\left(y_{1}+x_{1}+v\right)^{\prime}\left\{y_{1}+x_{1}\right\}, \quad x=\left(x_{1}, x_{2}\right) \in X, y=\left(y_{1}, y_{2}\right) \in Y,
$$


where for $\varphi \in V$, the operator $B(\varphi) \in \mathcal{L}\left(Q, V^{*}\right)$ and its Banach space adjoint, $B(\varphi)^{\prime} \in \mathcal{L}(V, Q)$ are given in (2.37) and (2.38), respectively.

The hypotheses of the theorem clearly imply that $\mathcal{F}(0,0)=0$, and that $\mathcal{F} \in C(X \times Y, Z)$; that is, $\mathcal{F}$ is a continuous mapping from $X \times Y$ into $Z$. We show next that $D_{y} \mathcal{F}(0,0)=$ $\left(D_{y} \mathcal{F}_{1}(0,0), D_{y} \mathcal{F}_{2}(0,0)\right)$ is a linear homeomorphism of $Y$ onto $Z$. We do this by demonstrating that for each $z=\left(z_{1}, z_{2}\right) \in Z, z_{1} \in L_{2}\left(0, \infty ; V^{*}\right)$ and $z_{2} \in L_{1}(0, \infty ; Q)$, there exist a unique $y=\left(y_{1}, y_{2}\right) \in Y, y_{1} \in L_{2}(0, \infty ; V) \cap H_{L}^{1}\left(0, \infty ; V^{*}\right)$ and $y_{2} \in L_{\infty}(0, \infty ; Q) \cap W_{L}^{1,1}(0, \infty ; Q)$ satisfying the linear initial value problem

$$
\begin{gathered}
D_{t} y_{1}+A_{0} y_{1}-B(v) y_{2}=z_{1} \quad t>0, \\
D_{t} y_{2}+B(v)^{\prime} y_{1}=z_{2}, \quad t>0, \\
y_{1}(0)=0 \quad \text { and } \quad y_{2}(0)=0,
\end{gathered}
$$

and by providing estimates which establish the continuous dependence of $y$ on $z$. If we assume that $V$ is separable, then the argument establishing the existence of a unique solution to the system (2.71)-(2.73) is the same as the one used to prove Theorem III.1.2 in [26]. Galerkin approximation is used to define a sequence of finite dimensional initial value problems which approximate the system (2.71)-(2.73). Of course each of the finite dimensional systems admits a unique solution, $y^{n}=\left(y_{1}^{n}, y_{2}^{n}\right)$. One then argues that these approximating solutions lie in a bounded subset of $Y$, that $y^{n} \rightarrow y$, weakly in $Y$, and that $y$ is the unique solution to the initial value problem (2.71)-(2.73). The key step in the proof depends upon the estimate for $\|\left. y\right|_{Y}$ in terms of $\|z\|_{Z}$ which we now derive. This estimate, which is given in (2.78) below, establishes the continuous dependence of $y$ on $z$ as well.

Taking the inner product of (2.71) with $y_{1}$ and (2.72) with $y_{2}$, and then adding, we obtain

$$
\frac{1}{2}\left\{D_{t}\left|y_{1}\right|^{2}+\left|y_{2}\right|_{Q}^{2}\right\}+\left\langle A_{0} y_{1}, y_{1}\right\rangle=\left\langle z_{1}, y_{1}\right\rangle+\left\langle z_{2}, y_{2}\right\rangle_{Q} .
$$

For any $\varepsilon>0$, Assumption (A4) implies that

$$
\begin{aligned}
& \frac{1}{2}\left\{D_{t}\left|y_{1}\right|^{2}+\left|y_{2}\right|_{Q}^{2}\right\}+\rho_{0}|| y_{1} \|^{2} \\
& \quad \leq\left\|z_{1}\right\|\left\|_{*}\right\| y_{1} \|+\left|z_{2}\right|_{Q}\left|y_{2}\right|_{Q} \\
& \quad \leq \frac{1}{2 \varepsilon}\left\|z_{1}\right\|_{*}^{2}+\frac{\varepsilon}{2}\left\|y_{1}\right\|^{2}+\frac{1}{2}\left|z_{2}\right|_{Q}\left\{1+\left|y_{2}\right|_{Q}^{2}\right\} .
\end{aligned}
$$

Choosing $\varepsilon<2 \rho_{0}$, setting $c_{0}=2 \rho_{0}-\varepsilon>0$ and $c_{1}=1 / \varepsilon$, integrating (2.74) from 0 to $t$, and recalling $(2.73)$, we obtain

$$
\begin{aligned}
& \left|y_{1}(t)\right|^{2}+\left|y_{2}(t)\right|_{Q}^{2}+c_{0} \int_{0}^{t}\left\|y_{1}(s)\right\|^{2} d s \\
& \quad \leq c_{1} \int_{0}^{t}\left\|z_{1}(s)\right\|_{*}^{2} d s+\int_{0}^{t}\left|z_{2}(s)\right|_{Q} d s+\int_{0}^{t}\left|z_{2}(s)\right|_{Q}\left|y_{2}(s)\right|_{Q}^{2} d s \\
& \quad \leq c_{1}\|z\|_{Z}^{2}+|| z\|\|_{Z}+\int_{0}^{t}\left|z_{2}(s)\right|_{Q}\left|y_{2}(s)\right|_{Q}^{2} d s
\end{aligned}
$$

An application of the Generalized Gronwall inequality (see [14]) yields

$$
\left|y_{1}(t)\right|^{2}+\left|y_{2}(t)\right|_{Q}^{2}+c_{0} \int_{0}^{t}\left\|y_{1}(s)\right\|^{2} d s
$$




$$
\begin{aligned}
& \leq\left\{c_{1}\|z\|_{Z}^{2}+\|z\|_{Z}\right\}\left\{1+\int_{0}^{t}\left|z_{2}(s)\right|_{Q} e^{\int_{s}^{t}\left|z_{2}(\tau)\right|_{Q} d \tau} d s\right\} \\
& \leq\left\{c_{1}\|z\|_{Z}^{2}+\|z\|_{Z}\right\}\left\{1+\|z\|_{Z} e^{\|z\|_{Z}}\right\}, \quad t \geq 0 .
\end{aligned}
$$

Equation (2.71) and Assumptions ( $A 1),(A 3)$, and ( $A 11)$ yield

$$
\left\|D_{t} y_{1}(t)\right\|_{*} \leq\left\|z_{1}(t)\right\|_{*}+\alpha_{0}\left\|y_{1}(t)\right\|+\alpha_{1}\|v(t)\|\left|y_{2}(t)\right|_{Q}, \quad \text { a.e. } t>0 .
$$

Consequently there exists a constant $c_{2}=c_{2}\left(\|v\|_{L_{2}(0, \infty ; V)}\right)>0$ such that

$$
\left\|y_{1}\right\|_{H_{L}^{1}\left(0, \infty ; V^{*}\right)}^{2} \leq c_{2}\left\{\left\|z_{1}\right\|_{L_{2}\left(0, \infty ; V^{*}\right)}^{2}+\left\|y_{1}\right\|_{L_{2}(0, \infty ; V)}^{2}+\left|y_{2}\right|_{L_{\infty}(0, \infty ; Q)}^{2}\right\} .
$$

Similarly, (2.72) yields

$$
\left|D_{t} y_{2}(t)\right|_{Q} \leq\left|z_{2}(t)\right|_{Q}+\alpha_{1}\|v(t)\|\left\|y_{1}(t)\right\|, \quad \text { a.e. } t>0,
$$

and therefore that

$$
\left\|D_{t} y_{2}\right\|_{L_{1}(0, \infty ; Q)} \leq\left\|z_{2}\right\|_{L_{1}(0, \infty ; Q)}+\alpha_{1}\|v\|_{L_{2}(0, \infty ; V)}|| y_{1} \|_{L_{2}(0, \infty ; V)}
$$

Combining (2.75), (2.76), and (2.77), we obtain that

$$
\|y\|_{Y} \leq h\left(\|z\|_{Z}\right)
$$

where $h: \mathbf{R}^{+} \rightarrow \mathbf{R}^{+}$is continuous and monotone increasing.

The following estimates for the dependence on $z$ on $y$ can also be obtained. Once again, (2.71) and Assumptions ( $A 1),(A 3)$, and (A11) imply that

$$
\left\|z_{1}(t)\right\|_{*} \leq\left\|D_{t} y_{1}(t)\right\|_{*}+\alpha_{0}\left\|y_{1}(t)\right\|+\alpha_{1}\|v(t)\|\left|y_{2}(t)\right|_{Q}, \quad \text { a.e. } t>0,
$$

and therefore, that

$$
\left\|z_{1}\right\|_{L_{2}\left(0, \infty ; V^{*}\right)}^{2} \leq c_{2}\left\{\left\|y_{1}\right\|_{H_{L}^{1}\left(0, \infty ; V^{*}\right)}^{2}+\left\|y_{1}\right\|_{L_{2}(0, \infty ; V)}^{2}+\left|y_{2}\right|_{L_{\infty}(0, \infty ; Q)}^{2}\right\} .
$$

Also (2.72) yields

$$
\left|z_{2}(t)\right|_{Q} \leq\left|D_{t} y_{2}(t)\right|_{Q}+\alpha_{1}\|v(t)\|\left\|y_{1}(t)\right\|, \quad \text { a.e. } t>0
$$

and therefore that

$$
\left\|z_{2}\right\|_{L_{1}(0, \infty ; Q)} \leq\left\|D_{t} y_{2}\right\|_{L_{1}(0, \infty ; Q)}+\alpha_{1}\|v\|_{L_{2}(0, \infty ; V)}\left\|y_{1}\right\|_{L_{2}(0, \infty ; V)}
$$

Combining (2.79) and (2.80), we obtain that

$$
\|z\|_{Z} \leq h_{0}\|y\|_{Y}
$$

where $h_{0}>0$.

It follows from the Implicit Function Theorem that there exists a $C>0$, such that if $x_{0}=$ $\left(\epsilon_{0}, r_{0}\right) \in X$ satisfies (2.67), then there exists a unique $y=y\left(x_{0}\right)=\left(y_{1}\left(x_{0}\right), y_{2}\left(x_{0}\right)\right) \in Y$, which is continuously differentiable in $x_{0}$ and which satisfies $\mathcal{F}\left(x_{0}, y\right)=\left(\mathcal{F}_{1}\left(x_{0}, y\right), \mathcal{F}_{2}\left(x_{0}, y\right)\right)=0$. Setting $e=y_{1}+\epsilon_{0}$ and $r=y_{2}+r_{0}$, we obtain the desired result. $\square$

Under additional hypotheses a similar approach can used to obtain a somewhat stronger result providing $L_{\infty}$ estimates. 
Theorem 2.8 Suppose that Assumptions (A1)-(A5) and Assumption (A10) are satisfied. Suppose further that

(i) $D_{1}=\operatorname{Dom}\left(A_{1}(q)\right)$ is independent of $q \in Q, D_{1} \subset D_{0}$, and there exists $\gamma_{0}>0$ for which $\left|A_{1}(q) \varphi\right| \leq \gamma_{0}|q|_{Q}\left|A_{0} \varphi\right|, q \in Q$, and $\varphi \in D_{1}$,

(ii) $A_{0}$ is symmetric $\left(A_{0}: D_{0} \subset H \rightarrow H\right.$ is self adjoint),

(iii) $v \in L_{\infty}(0, \infty ; V), v(t) \in D_{0}$, a.e. $t>0, A_{0} v \in L_{2}(0, \infty ; H)$, and

(iv) $\epsilon_{0} \in D_{0}$.

Then there exists a constant $C>0$ such that if

$$
\left|A_{0} e_{0}\right|+\left|r_{0}\right|_{Q}<C,
$$

then the initial value problem (2.64)-(2.66) has a unique solution $(e, r)$ with $e \in L_{\infty}(0, \infty ; V) \cap$ $H^{1}(0, \infty ; H)$ and $r \in L_{\infty}(0, \infty ; Q) \cap W^{1,1}(0, \infty ; Q)$. Moreover, $e(t) \in D_{0}$, a.e. $t>0$, and $A_{0} e \in L_{2}(0, \infty ; H)$.

Proof. Let $D_{0}$ be endowed with the graph norm. Then the proof is completely analogous to the one given above for Theorem 2.7 based upon the Implicit Function Theorem. However in this case, we take the Banach spaces $X, Y$, and $Z$ to be

$$
\begin{gathered}
X=D_{0} \times Q, \\
Y=\left\{L_{2}\left(0, \infty ; D_{0}\right) \cap H_{L}^{1}(0, \infty ; H)\right\} \times\left\{L_{\infty}(0, \infty ; Q) \cap W_{L}^{1,1}(0, \infty ; Q)\right\},
\end{gathered}
$$

and

$$
Z=L_{2}(0, \infty ; H) \times L_{1}(0, \infty ; Q),
$$

respectively. The norms on these spaces are chosen analogously to (2.68), (2.69), and (2.70).

We note that conditions sufficient to guarantee that hypothesis (iii) in the statement of Theorem 2.8 above holds, are given in Theorem 2.1 (iv).

As an example of the kinds of systems to which the theory in this section applies, let $\Omega \subset \mathbf{R}^{n}$ be a bounded domain. Let $H=L_{2}(\Omega), V=H_{0}^{1}(\Omega)$, and let $Q$ be a closed subspace of $H^{s}(\Omega)^{n^{2}} \times H^{s}(\Omega)^{n} \times H^{s}(\Omega)$ with $s>n / 2$. Let $\hat{V}^{*}=V^{*}=H^{-1}(\Omega)$. Let $A_{0} \in \mathcal{L}\left(V, V^{*}\right)$ be given by

$$
A_{0} \varphi=-\sum_{i, j=1}^{n} D_{j}\left\{a_{i, j} D_{i} \varphi\right\}+\sum_{i=1}^{n} b_{i} D_{i} \varphi+c \varphi, \quad \varphi \in V .
$$

where $a_{i, j} \in L_{\infty}(\Omega), a_{i, j}(x)=a_{j, i}(x)$, a.e. $x \in \Omega, i, j=1,2, \ldots, n$,

$$
\rho_{0}|\xi|^{2} \leq \sum_{i, j=1}^{n} a_{i, j}(x) \xi_{i} \xi_{j} \leq \rho_{1}|\xi|^{2}, \quad \xi \in \mathbf{R}^{n} \text {, a.e. } x \in \Omega,
$$

for some constants $\rho_{0}, \rho_{1}>0, b_{i} \in W^{1, \frac{n}{2}}(\Omega), i=1,2, \ldots, n$ with

$$
\sum_{i=1}^{n} D_{i} b_{i}(x) \leq 0, \quad \text { a.e. } x \in \Omega,
$$

and $c \in L^{\frac{n}{2}}(\Omega)$ with $c(x) \geq 0$, a.e. $x \in \Omega$. 
For $q=\left(\left\{q_{i, j}\right\},\left\{q_{i}\right\}, q_{0}\right) \in Q$, let $A_{1}(q) \in \mathcal{L}\left(V, V^{*}\right)$ be given by

$$
A_{1}(q) \varphi=-\sum_{i, j=1}^{n} D_{j}\left\{q_{i, j} D_{i} \varphi\right\}+\sum_{i=1}^{n} q_{i} D_{i} \varphi+q_{0} \varphi, \quad \varphi \in V .
$$

Note that these are not the most general conditions possible to guarantee that Assumptions $(A 1)-(A 5)$ hold for the general class of second order elliptic plants and reference models.

\section{Tracking and Parameter Error Convergence}

In this section we argue that the control objective is achieved (i.e. that the tracking error, $\epsilon(t)$, converges to zero as $t \rightarrow \infty$ and that the feedback control, $f$, is, in some sense, bounded), and that under an additional richness condition on the reference model, parameter convergence is obtained (i.e. that $q(t) \rightarrow \bar{q}$ as $t \rightarrow \infty$ ). We require that our standing assumptions (A1)(A5) continue to hold, and that the error equations, (2.32)-(2.35), admit a unique solution $(e, v, r)$, with $\epsilon, v \in L_{2}(0, T ; V) \cap C([0, T] ; H), D_{t} e, D_{t} v \in L_{2}\left(0, T ; V^{*}\right), r \in C([0, T] ; Q)$, and $D_{t} r \in L_{2}(0, T ; Q)$, for all $T>0$.

Define $E:[0, \infty) \rightarrow \mathbf{R}^{+}$by

$$
E(t)=\frac{1}{2}\left\{|\epsilon(t)|^{2}+|r(t)|_{Q}^{2}\right\}, \quad t \geq 0 .
$$

Lemma 3.1 For $(e, v, r)$ the solution to the initial value problem (2.32)-(2.35), the function $E:[0, \infty) \rightarrow \mathbf{R}^{+}$given by (3.1) is non-increasing, and we have that

$$
E(t)+\rho_{0} \int_{0}^{t}\|e(s)\|^{2} d s \leq \xi_{0}, \quad t \geq 0,
$$

where $\xi_{0}=E(0)=\frac{1}{2}\left\{\left|e_{0}\right|^{2}+\left|r_{0}\right|_{Q}^{2}\right\}$.

Proof. Using (2.32), (2.34), and Assumption (A4), we obtain

$$
\begin{aligned}
D_{t} E(t) & =\left\langle D_{t} e(t), \epsilon(t)\right\rangle+\left\langle D_{t} r(t), r(t)\right\rangle_{Q} \\
& =-\left\langle A_{0} e(t), \epsilon(t)\right\rangle \\
& \leq-\rho_{0}\|e(t)\|^{2}, \quad \text { a.e. } t>0 .
\end{aligned}
$$

The estimate in (3.3) implies that $E$ is non-increasing. Integrating this expression from 0 to $t$, $t>0$, we obtain the result given in (3.2).

The above lemma yields the following immediate corollary.

Corollary 3.2 For $(\epsilon, v, r)$ the solution to the initial value problem (2.32)-(2.35), we have $\epsilon \in$ $L_{\infty}(0, \infty ; H) \cap L_{2}(0, \infty ; V)$ and $r \in L_{\infty}(0, \infty ; Q)$.

Lemma 3.3 Let $(e, v, r)$ be the solution to the initial value problem (2.32)-(2.35). Then, if $g \in L_{\infty}(0, \infty ; H)$, it follows that

$$
\int_{t_{1}}^{t_{2}}\|v(t)\|^{2} d t \leq c_{1}+c_{2}\left(t_{2}-t_{1}\right)
$$

for all $t_{2} \geq t_{1} \geq 0$, where $c_{1}$ and $c_{2}$ are positive constants which do not depend on $t_{1}$ and $t_{2}$. 
Proof. It follows from (2.9) and (2.11) that if $g \in L_{\infty}(0, \infty ; H)$ then $v \in L_{\infty}(0, \infty ; H)$. Then, integrating (2.14) from $t_{1}$ to $t_{2}$, we obtain

$$
\left|v\left(t_{2}\right)\right|^{2}+\rho_{0} \int_{t_{1}}^{t_{2}}\|v(t)\|^{2} d t \leq\left|v\left(t_{1}\right)\right|^{2}+\int_{t_{1}}^{t_{2}}\|g(t)\|_{*}^{2} d t
$$

Recalling (2.1), it follows from (3.4) that

$$
\int_{t_{1}}^{t_{2}} \|\left. v(t)\right|^{2} d t \leq \frac{1}{\rho_{0}}|v|_{L_{\infty}(0, \infty ; H)}^{2}+\frac{K}{\rho_{0}}|g|_{L_{\infty}(0, \infty ; H)}^{2}\left(t_{2}-t_{1}\right)
$$

In the theorem that follows, we establish that the desired control objective is achieved. The proof we provide is in the spirit of the argument used to verify Barbălat's Lemma in [33].

Theorem 3.4 For $(e, v, r)$ the solution to the initial value problem (2.32)-(2.35) and $f$ the adaptive feedback control law given by (2.23) or (2.24), we have the following results.

(i) If $g \in L_{2}\left(0, \infty ; V^{*}\right) \cup L_{\infty}(0, \infty ; H)$, then $\lim _{t \rightarrow \infty}|e(t)|=0$.

(ii) If $g \in L_{2}\left(0, \infty ; V^{*}\right)$, then $u \in L_{\infty}(0, \infty ; H) \cap L_{2}(0, \infty ; V)$ and $f \in L_{2}\left(0, \infty ; V^{*}\right)$.

(iii) If the operator $A_{0}$ is symmetric in the sense of (2.13), $u_{0}, v_{0} \in V, g \in L_{2}(0, \infty ; H)$ and satisfies (2.12), and for $\varphi \in D_{0}$ and $q \in Q$, we have $A(q) \varphi \in H$ and

$$
\left|A_{1}(q) \varphi\right| \leq \gamma_{1}|q|_{Q}\left|A_{0} \varphi\right|
$$

for some $\gamma_{1}>0$ for which $\gamma_{1}|r|_{L_{\infty}(0, \infty ; Q)}<1$, then $u(t) \in D_{0}$, a.e. $t>0, u \in L_{\infty}(0, \infty ; V)$, $A_{0} u \in L_{2}(0, \infty ; H)$. If, in addition,

(a) $g \in L_{\infty}\left(0, \infty ; V^{*}\right)$, then $f \in L_{\infty}\left(0, \infty ; V^{*}\right)$,

or if, in addition,

(b) for $\varphi \in D_{0}$ we have $A_{2} \varphi \in H$ and $\left|A_{2} \varphi\right| \leq \gamma_{2}\left|A_{0} \varphi\right|$, for some $\gamma_{2}>0$, then $f \in$ $L_{2}(0, \infty ; H)$.

Proof. Let $t_{2} \geq t_{1} \geq 0$ and note that Assumptions (A1) and (A4), (2.32), and Lemma 3.1 imply that

$$
\begin{aligned}
\left|e\left(t_{2}\right)\right|^{2}-\left|e\left(t_{1}\right)\right|^{2} & =\int_{t_{1}}^{t_{2}} \frac{d}{d t}|e(t)|^{2} d t \\
& =2 \int_{t_{1}}^{t_{2}}\left\langle D_{t} \epsilon(t), e(t)\right\rangle d t \\
& =-2 \int_{t_{1}}^{t_{2}}\left\langle A_{0} e(t), e(t)\right\rangle d t-2 \int_{t_{1}}^{t_{2}}\left\langle A_{1}(r(t))\{e(t)+v(t)\}, e(t)\right\rangle d t \\
& \leq-2 \rho_{0} \int_{t_{1}}^{t_{2}}|e(t)|^{2} d t+2 \alpha_{1} \int_{t_{1}}^{t_{2}}|r(t)|_{Q}\{\|e(t)\|+\mid v(t) \|\}|e(t)| d t \\
& \leq 2 \alpha_{1} \int_{t_{1}}^{t_{2}}\{\|e(t)\|+\|v(t)\|\} E(t) d t \\
& \leq 2 \alpha_{1} \xi_{0}\left(t_{2}-t_{1}\right)^{\frac{1}{2}}\left(\int_{t_{1}}^{t_{2}}\{\|e(t)\|+\|v(t)\|\}^{2} d t\right)^{\frac{1}{2}} \\
& \leq 2 \sqrt{2} \alpha_{1} \xi_{0}\left(t_{2}-t_{1}\right)^{\frac{1}{2}}\left(\int_{t_{1}}^{t_{2}}\|e(t)\|^{2} d t+\int_{t_{1}}^{t_{2}}\|v(t)\|^{2} d t\right)^{\frac{1}{2}} .
\end{aligned}
$$


If $g \in L_{2}\left(0, \infty ; V^{*}\right)$, then Theorem 2.1 implies that

$$
\int_{t_{1}}^{t_{2}}\|v(t)\|^{2} d t \leq \zeta_{0}
$$

for some $\zeta_{0}>0$, for all $t_{2} \geq t_{1} \geq 0$. It then follows from Lemma 3.1 and (3.5) that

$$
\left|e\left(t_{2}\right)\right|^{2}-\left|e\left(t_{1}\right)\right|^{2} \leq \kappa_{0}\left(t_{2}-t_{1}\right)^{\frac{1}{2}},
$$

where $\kappa_{0}=2 \sqrt{2} \alpha_{1} \xi_{0}\left\{\frac{\xi_{0}}{\rho_{0}}+\zeta_{0}\right\}^{\frac{1}{2}}$. On the other hand, if $g \in L_{\infty}(0, \infty ; H)$, then Lemma 3.1, Lemma 3.3, and (3.5) imply that

$$
\left|e\left(t_{2}\right)\right|^{2}-\left|e\left(t_{1}\right)\right|^{2} \leq \kappa_{1}\left(t_{2}-t_{1}\right)^{\frac{1}{2}}+\kappa_{2}\left(t_{2}-t_{1}\right),
$$

where $\kappa_{1}=2 \sqrt{2} \alpha_{1} \xi_{0}\left\{\frac{\xi_{0}}{\rho_{0}}+c_{1}\right\}^{\frac{1}{2}}$ and $\kappa_{2}=2 \sqrt{2} \alpha_{1} \xi_{0} \sqrt{c_{2}}$.

Now suppose that $\lim _{t \rightarrow \infty}|e(t)| \neq 0$. Then there exist $\varepsilon>0$ and a sequence $\left\{t_{i}\right\}_{i=1}^{\infty}$ with $\lim _{i \rightarrow \infty} t_{i}=\infty$ for which

$$
\left|\epsilon\left(t_{i}\right)\right|^{2}>\varepsilon, \quad i=1,2, \ldots
$$

If $g \in L_{2}\left(0, \infty ; V^{*}\right)$, then (3.6) and (3.8) imply that for $\delta>0$ and $i=1,2, \ldots$, we have

$$
\begin{aligned}
\int_{t_{i}-\delta}^{t_{i}}|e(t)|^{2} d t & =\int_{t_{i}-\delta}^{t_{i}}\left|e\left(t_{i}\right)\right|^{2} d t-\int_{t_{i}-\delta}^{t_{i}}\left\{\left|e\left(t_{i}\right)\right|^{2}-|e(t)|^{2}\right\} d t \\
& >\varepsilon \delta-\kappa_{0} \int_{t_{i}-\delta}^{t_{i}}\left(t_{i}-t\right)^{\frac{1}{2}} d t \\
& =\varepsilon \delta-\frac{2}{3} \kappa_{0} \delta^{\frac{3}{2}} .
\end{aligned}
$$

Choosing $\delta=\frac{9 \varepsilon}{16 \kappa_{0}^{2}}$, and replacing the sequence $\left\{t_{i}\right\}_{i=1}^{\infty}$ by a subsequence, $\left\{t_{i_{j}}\right\}_{j=1}^{\infty}$, for which $t_{i_{j+1}}-t_{i_{j}}>\delta, j=1,2, \ldots$, we obtain

$$
\int_{t_{i_{j}}-\delta}^{t_{i_{j}}}|e(t)|^{2} d t \geq \frac{\varepsilon \delta}{2}, \quad j=1,2, \ldots
$$

If $g \in L_{\infty}(0, \infty ; H)$, then (3.7) and (3.8) imply that for $\delta>0$ and $i=1,2, \ldots$, we have

$$
\begin{aligned}
\int_{t_{i}-\delta}^{t_{i}}|e(t)|^{2} d t & =\int_{t_{i}-\delta}^{t_{i}}\left|e\left(t_{i}\right)\right|^{2} d t-\int_{t_{i}-\delta}^{t_{i}}\left\{\left|e\left(t_{i}\right)\right|^{2}-|e(t)|^{2}\right\} d t \\
& >\varepsilon \delta-\int_{t_{i}-\delta}^{t_{i}}\left\{\kappa_{1}\left(t_{i}-t\right)^{\frac{1}{2}}+\kappa_{2}\left(t_{i}-t\right)\right\} d t \\
& =\varepsilon \delta-\frac{2}{3} \kappa_{1} \delta^{\frac{3}{2}}-\frac{1}{2} \kappa_{2} \delta^{2} .
\end{aligned}
$$

Choosing

$$
\delta=\left(\sqrt{\frac{4}{9}\left(\frac{\kappa_{1}}{\kappa_{2}}\right)^{2}+\frac{\varepsilon}{\kappa_{2}}}-\frac{2}{3}\left(\frac{\kappa_{1}}{\kappa_{2}}\right)\right)^{2},
$$

we once again obtain (3.9). But (3.9) contradicts the fact that Lemma 3.1 implies that

$$
\int_{0}^{\infty}|\epsilon(t)|^{2} d t \leq K^{2} \int_{0}^{\infty} \|\left.\epsilon(t)\right|^{2} d t \leq K^{2} \frac{\xi_{0}}{\rho_{0}}<\infty
$$


Consequently, $\lim _{t \rightarrow \infty}|e(t)|^{2}=0$, and therefore, $\lim _{t \rightarrow \infty}|e(t)|=0$, which establishes $(i)$.

Corollary 3.2 above implies that $e \in L_{\infty}(0, \infty ; H) \cap L_{2}(0, \infty ; V)$, and if $g \in L_{2}\left(0, \infty ; V^{*}\right)$, then Theorem 2.1 implies that $v \in L_{\infty}(0, \infty ; H) \cap L_{2}(0, \infty ; V)$ as well. Consequently, it follows that $u=e+v \in L_{\infty}(0, \infty ; H) \cap L_{2}(0, \infty ; V)$. To establish that $f \in L_{2}\left(0, T ; V^{*}\right)$, we note that (2.24), Assumptions (A1) and (A3), and (2.31) imply that for a.e. $t>0$

$$
\begin{aligned}
\|f(t)\|_{*} & =\sup _{\|\varphi\| \leq 1}|\langle f(t), \varphi\rangle| \\
& =\sup _{\|\varphi\| \leq 1}\left|\left\langle A(q(t)) u(t)-A_{0} u(t)+g(t), \varphi\right\rangle\right| \\
& =\sup _{\|\varphi\| \leq 1}\left|\left\langle A_{1}(q(t)) u(t)+A_{2} u(t)-A_{0} u(t)+g(t), \varphi\right\rangle\right| \\
& \leq\left\{\alpha_{1}|q(t)|_{Q}+\alpha_{2}+\alpha_{0}\right\}\|u(t)\|+\|g(t)\|_{*} \\
& \leq\left\{\alpha_{1}\left\{|r(t)|_{Q}+|\bar{q}|_{Q}\right\}+\alpha_{2}+\alpha_{0}\right\}\|u(t)\|+\mid g(t) \|_{*} \\
& \leq \sigma\|u(t)\|+\mid g(t) \|_{*},
\end{aligned}
$$

where $\sigma=\alpha_{1}\left\{\sqrt{\xi_{0}}+|\bar{q}|_{Q}\right\}+\alpha_{2}+\alpha_{0}$. It follows that

$$
\|f(t)\|_{*}^{2} \leq 2 \sigma^{2}\|u(t)\|^{2}+2\|g(t)\|_{*}^{2}, \quad \text { a.e. } t>0 .
$$

The fact that $g \in L_{2}\left(0, \infty ; V^{*}\right)$ and $u \in L_{2}(0, \infty ; V)$ immediately yields (ii).

To establish (iii), we first note that under the present assumptions, Theorem 2.1 implies that $v \in L_{\infty}(0, \infty ; V), v(t) \in D_{0}$, a.e. $t>0$, and $A_{0} v \in L_{2}(0, \infty ; H)$. Also, Corollary 3.2 implies that $r \in L_{\infty}(0, \infty ; Q)$. Now, recalling the definition of the norm $\|\cdot\|_{0}$ on $V$ from Section 2, (2.32) with $\varphi=A_{0} \epsilon(t)$ implies that

$$
\left\langle D_{t} e(t), A_{0} e(t)\right\rangle+\left|A_{0} e(t)\right|^{2}=\left\langle A_{1}(r(t))\{e(t)+v(t)\}, A_{0} e(t)\right\rangle, \quad \text { a.e. } t>0,
$$

and therefore, for any $\varepsilon>0$, that

$$
\begin{aligned}
\left.\frac{1}{2} D_{t}|| \epsilon(t)\right|_{0} ^{2}+\left|A_{0} e(t)\right|^{2} \leq & \left|A_{1}(r(t))\{\epsilon(t)+v(t)\}\right|\left|A_{0} \epsilon(t)\right| \\
\leq & \gamma_{1}|r(t)|_{Q}\left|A_{0} e(t)+A_{0} v(t)\right|\left|A_{0} e(t)\right| \\
\leq & \gamma_{1}|r|_{L_{\infty}(0, \infty ; Q)}\left|A_{0} e(t)\right|^{2}+\gamma_{1}|r|_{L_{\infty}(0, \infty ; Q)} \frac{\varepsilon}{2}\left|A_{0} e(t)\right|^{2} \\
& \quad+\gamma_{1}|r|_{L_{\infty}(0, \infty ; Q)} \frac{1}{2 \varepsilon}\left|A_{0} v(t)\right|^{2}, \quad \text { a.e. } t>0 .
\end{aligned}
$$

Integrating (3.11) from 0 to $t$, recalling (2.35), (2.15), and our assumption that $\gamma_{1}|r|_{L_{\infty}(0, \infty ; Q)}<$ 1 , and choosing $\varepsilon>0$ sufficiently small, we find that

$$
\rho_{0}\|e(t)\|^{2}+\sigma_{0} \int_{0}^{t}\left|A_{0} e(s)\right|^{2} d s \leq \alpha_{0}\left\|e_{0}\right\|^{2}+\gamma_{1}|r|_{L_{\infty}(0, \infty ; Q)} \frac{1}{2 \varepsilon} \int_{0}^{t}\left|A_{0} v(s)\right|^{2} d s \quad t \geq 0,
$$

for some $\sigma_{0}>0$. It follows from (3.12) that $e \in L_{\infty}(0, \infty ; V), e(t) \in D_{0}$, a.e. $t>0$, and $A_{0} \epsilon \in L_{2}(0, \infty ; H)$. Consequently, $u=\epsilon+v \in L_{\infty}(0, \infty ; V), u(t) \in D_{0}$, a.e. $t>0$, and $A_{0} u \in L_{2}(0, \infty ; H)$. This, together with (3.10) establishes the claim in $(a)$. To establish the claim in $(b)$, we have the estimate

$$
|f(t)|=\sup _{|\varphi| \leq 1}|\langle f(t), \varphi\rangle|
$$




$$
\begin{aligned}
& =\sup _{|\varphi| \leq 1}\left|\left\langle A(q(t)) u(t)-A_{0} u(t)+g(t), \varphi\right\rangle\right| \\
& =\sup _{|\varphi| \leq 1}\left|\left\langle A_{1}(q(t)) u(t)+A_{2} u(t)-A_{0} u(t)+g(t), \varphi\right\rangle\right| \\
& \leq\left\{\gamma_{1}|q(t)|_{Q}+\gamma_{2}+1\right\}\left|A_{0} u(t)\right|+|g(t)| \\
& \leq\left\{\gamma_{1}\left\{|r|_{L_{\infty}(0, \infty ; Q)}+|\bar{q}|_{Q}\right\}+\gamma_{2}+1\right\}\left|A_{0} u(t)\right|+|g(t)|, \quad \text { a.e. } t>0,
\end{aligned}
$$

from which the desired result immediately follows.

We note that the condition that $\gamma_{1}|r|_{L_{\infty}(0, \infty ; Q)}<1$ can be satisfied with an appropriate choice of the reference model dynamics, $A_{0}$, the initial estimate of the unknown parameters, $q_{0}$, (i.e. that it be sufficiently close to the true parameters, $\bar{q}$, and the initial state of the reference model, $v_{0}$, (i.e. that it be sufficiently close to the initial state of the plant, $u_{0}$ ). The last two sufficient conditions are a consequence of Lemma 3.1 .

In addition to meeting the designated control objective, it is also desirable to have an adaptive control scheme provide parameter convergence as well. In order to establish that the scheme we consider here yields convergence of the parameters, $q(t)$, to the true parameters, $\bar{q}$, as $t \rightarrow \infty$, we require the following additional richness condition on the reference model.

Definition 3.5 The reference model, (2.7), (2.8), or the triple, $\left\{A_{0}, g, v_{0}\right\}$, consisting of the reference model dynamics operator $A_{0}$, the input reference signal, $g$, and the initial state of the reference model, $v_{0}$, will be said to be persistently exciting, or, sufficiently rich, if there exist positive constants $\tau_{0}, \delta_{0}$, and $\varepsilon_{0}$, such that for each $p \in Q$ with $|p|_{Q}=1$ and $t \geq 0$ sufficiently large, there exists $\tilde{t} \in\left[t, t+\tau_{0}\right]$ for which

$$
\left\|\int_{\tilde{t}}^{\tilde{t}+\delta_{0}} A_{1}(p) u(\tau) d \tau\right\|_{*} \geq \varepsilon_{0}
$$

where $u$ is the closed loop state of the plant as given by the system (2.27)-(2.30).

Theorem 3.6 If either $g \in L_{2}\left(0, \infty ; V^{*}\right)$ or $g \in L_{\infty}(0, \infty ; V)$ and $v_{0} \in V$, and if the reference model, (2.7),(2.8), is persistently exciting, then $\lim _{t \rightarrow \infty}|r(t)|_{Q}=0$.

Proof. If $g \in L_{2}\left(0, \infty ; V^{*}\right)$, then Theorem 3.4 implies that $u \in L_{2}(0, \infty ; V)$. Corollary $3.2 \mathrm{im}-$ plies that $r \in L_{\infty}(0, \infty ; Q)$, and Lemma 3.1 together with Theorem 3.4 imply that $\lim _{t \rightarrow \infty}|r(t)|_{Q}$ exists. If we assume that $\lim _{t \rightarrow \infty}|r(t)|_{Q} \neq 0$, then there exists $\left\{t_{k}\right\}_{k=1}^{\infty}$, an increasing sequence of positive numbers for which $\lim _{k \rightarrow \infty} t_{k}=\infty$ and

$$
\left|r\left(t_{k}\right)\right|_{Q} \geq \delta, \quad k=1,2, \ldots,
$$

for some $\delta>0$. If the reference model, (2.7),(2.8) is persistently exciting, it then follows from Assumption $(A 1)$ that for each $k=1,2 \ldots$ and some $\tilde{t}_{k} \in\left[t_{k}, t_{k}+\tau_{0}\right]$, we have

$$
\begin{aligned}
0<\delta \varepsilon_{0} & \leq\left|r\left(t_{k}\right)\right|_{Q}\left\|\int_{\tilde{t}_{k}}^{\tilde{t}_{k}+\delta_{0}} A_{1}\left(\frac{r\left(t_{k}\right)}{\left|r\left(t_{k}\right)\right|_{Q}}\right) u(t) d t\right\|_{*} \\
& =\| \int_{\tilde{t}_{k}}^{\tilde{t}_{k}+\delta_{0}} A_{1}\left(r\left(t_{k}\right) u(t) d t \|_{*}\right. \\
& \leq \alpha_{1}|r|_{L_{\infty}(0, \infty ; Q)} \sqrt{\delta_{0}}\left\{\int_{\tilde{t}_{k}}^{\tilde{t}_{k}+\delta_{0}}\|u(t)\|^{2} d t\right\}^{\frac{1}{2}} .
\end{aligned}
$$


Letting $k \rightarrow \infty$ in (3.14), and using the fact that $u \in L_{2}(0, \infty ; V)$ implies that

$$
\lim _{k \rightarrow \infty} \int_{\tilde{t}_{k}}^{\tilde{t}_{k}+\delta_{0}}\|u(t)\|^{2} d t=0
$$

we obtain a contradiction.

Now suppose that $g \in L_{\infty}(0, \infty ; V)$ and $v_{0} \in V$. We first recall that Theorem 2.1 implies that $v \in L_{\infty}(0, \infty ; V)$. Now, for $t_{2}>t_{1},(2.32)$, Assumption (A3), and (2.1) imply that

$$
\begin{aligned}
\left\|\int_{t_{1}}^{t_{2}} A_{1}(r(t)) u(t) d t\right\|_{*} & =\left\|\int_{t_{1}}^{t_{2}} A_{1}(r(t))\{\epsilon(t)+v(t)\} d t\right\|_{*} \\
& \leq\left\|e\left(t_{2}\right)\right\|_{*}+\left\|e\left(t_{1}\right)\right\|_{*}+\int_{t_{1}}^{t_{2}}\left\|A_{0} e(t)\right\|_{*} d t \\
& \leq K\left|e\left(t_{2}\right)\right|+K\left|e\left(t_{1}\right)\right|+\alpha_{0}\left(t_{2}-t_{1}\right)^{\frac{1}{2}}\left\{\int_{t_{1}}^{t_{2}}\|e(t)\|^{2} d t\right\}^{\frac{1}{2}} .
\end{aligned}
$$

Also, from (2.34), Assumption (A1), and Lemma 3.1 it follows that

$$
\begin{aligned}
\left|r\left(t_{2}\right)-r\left(t_{1}\right)\right|_{Q} & =\sup _{|p|_{Q} \leq 1}\left|\left\langle r\left(t_{2}\right)-r\left(t_{1}\right), p\right\rangle_{Q}\right| \\
& =\sup _{|p|_{Q} \leq 1}\left|\left\langle\int_{t_{1}}^{t_{2}} D_{t} r(t) d t, p\right\rangle_{Q}\right| \\
& \leq \int_{t_{1}}^{t_{2}} \sup _{|p|_{Q} \leq 1}\left|\left\langle A_{1}(p)\{e(t)+v(t)\}, e(t)\right\rangle\right| d t \\
& \leq \alpha_{1} \int_{t_{1}}^{t_{2}}\{|\epsilon(t)|+\mid v(t) \|\}\|e(t)\| d t \\
& \leq \alpha_{1} \int_{t_{1}}^{t_{2}}\|e(t)\|^{2} d t+\alpha_{1}\|v\|_{L_{\infty}(0, \infty ; V)} \int_{t_{1}}^{t_{2}}\|e(t)\| d t \\
& \leq \alpha_{1} \int_{t_{1}}^{t_{2}}\|e(t)\|^{2} d t+\alpha_{1}\|v\|_{L_{\infty}(0, \infty ; V)}\left(t_{2}-t_{1}\right)^{\frac{1}{2}}\left\{\int_{t_{1}}^{t_{2}}\|e(t)\|^{2} d t\right\}^{\frac{1}{2}} .
\end{aligned}
$$

Once again assume that $\lim _{t \rightarrow \infty}|r(t)|_{Q} \neq 0$, and let $\left\{t_{k}\right\}_{k=1}^{\infty}$ be an increasing sequence of positive numbers for which $\lim _{k \rightarrow \infty} t_{k}=\infty$ and for which (3.13) holds for some $\delta>0$. Assume further that the reference model, (2.7),(2.8), is persistently exciting, and for each $k=1,2, \ldots$, let $\tilde{t}_{k} \in\left[t_{k}, t_{k}+\tau_{0}\right]$ be such that

$$
\left\|\int_{\tilde{t}_{k}}^{\tilde{t}_{k}+\delta_{0}} A_{1}\left(\frac{r\left(t_{k}\right)}{\left|r\left(t_{k}\right)\right|_{Q}}\right) u(t) d t\right\|_{*} \geq \varepsilon_{0} .
$$

Then, using (3.13), (3.16), (3.17), (3.18), and Assumptions (A1) and (A2), we obtain the estimate

$$
\begin{aligned}
0< & \delta \varepsilon_{0} \leq\left|r\left(t_{k}\right)\right|_{Q}\left\|\int_{\tilde{t}_{k}}^{\tilde{t}_{k}+\delta_{0}} A_{1}\left(\frac{r\left(t_{k}\right)}{\left|r\left(t_{k}\right)\right|_{Q}}\right) u(t) d t\right\|_{*}=\left\|\int_{\tilde{t}_{k}}^{\tilde{t}_{k}+\delta_{0}} A_{1}\left(r\left(t_{k}\right)\right) u(t) d t\right\|_{*} \\
\leq & \left\|\int_{\tilde{t}_{k}}^{\tilde{t}_{k}+\delta_{0}} A_{1}(r(t)) u(t) d t\right\|_{*}+\left\|\int_{\tilde{t}_{k}}^{\tilde{t}_{k}+\delta_{0}} A_{1}\left(r\left(t_{k}\right)-r(t)\right)\{e(t)+v(t)\} d t\right\|_{*} \\
& \leq K\left|e\left(\tilde{t}_{k}+\delta_{0}\right)\right|+K\left|e\left(\tilde{t}_{k}\right)\right|+\alpha_{0} \sqrt{\delta_{0}}\left\{\int_{\tilde{t}_{k}}^{\tilde{t}_{k}+\delta_{0}} \|\left. e(t)\right|^{2} d t\right\}^{\frac{1}{2}}
\end{aligned}
$$




$$
\begin{aligned}
&+\alpha_{1} \int_{\tilde{t}_{k}}^{\tilde{t}_{k}+\delta_{0}}\left|r(t)-r\left(t_{k}\right)\right|_{Q}\{\|e(t)\|+\| v(t) \mid\} d t \\
& \leq K\left|e\left(\tilde{t}_{k}+\delta_{0}\right)\right|+K\left|e\left(\tilde{t}_{k}\right)\right|+\alpha_{0} \sqrt{\delta_{0}}\left\{\int_{\tilde{t}_{k}}^{\tilde{t}_{k}+\delta_{0}} \|\left. e(t)\right|^{2} d t\right\}^{\frac{1}{2}} \\
&+\alpha_{1}^{2}\left(\int_{\tilde{t}_{k}}^{\tilde{t}_{k}+\tau_{0}+\delta_{0}}\|e(t)\|^{2} d t+\|\left. v\right|_{L_{\infty}(0, \infty ; V)} \sqrt{\tau_{0}+\delta_{0}}\left\{\int_{\tilde{t}_{k}}^{\tilde{t}_{k}+\tau_{0}+\delta_{0}} \|\left.\epsilon(t)\right|^{2} d t\right\}^{\frac{1}{2}}\right) \\
& \times \int_{\tilde{t}_{k}}^{\tilde{t}_{k}+\delta_{0}}\{\|e(t)\|+\|v(t)\|\} d t \\
&+\alpha_{1}^{2}\left(\int_{\tilde{t}_{k}}^{\tilde{t}_{k}+\tau_{0}+\delta_{0}}\|e(t)\|^{2} d t+\|v\|_{L_{\infty}(0, \infty ; V)} \sqrt{\tau_{0}+\delta_{0}}\left\{\int_{\tilde{t}_{k}}^{\tilde{t}_{k}+\tau_{0}+\delta_{0}} \|\left.\epsilon(t)\right|^{2} d t\right\}^{\frac{1}{2}}\right) \\
& \\
& \times\left(\sqrt{\delta_{0}}\left\{\int_{\tilde{t}_{k}}^{\tilde{t}_{k}+\delta_{0}}\|e(t)\|^{2} d t\right\}^{\frac{1}{2}}+\int_{\tilde{t}_{k}} \tilde{t}_{k}\|v(t)\|_{L_{\infty}(0, \infty ; V)}\right) .
\end{aligned}
$$

Now Lemma 3.1 implies that for any $L>0$

$$
\lim _{t \rightarrow \infty} \int_{t}^{t+L}\|e(s)\|^{2} d s=0 .
$$

Therefore, letting $k \rightarrow \infty$ in (3.19), Lemma 3.1 and Theorem 3.4 imply that

$$
\begin{aligned}
0< & \delta \varepsilon_{0} \\
\leq & \left.K \lim _{k \rightarrow \infty}\left|e\left(\tilde{t}_{k}+\delta_{0}\right)\right|+K \lim _{k \rightarrow \infty}\left|e\left(\tilde{t}_{k}\right)\right|+\alpha_{0} \sqrt{\delta_{0}} \lim _{k \rightarrow \infty}\left\{\int_{t_{k}}^{t_{k}+\tau_{0}+\delta_{0}}\|e(t)\|^{2} d t\right\}^{\frac{1}{2}}\|(t)\|^{2} d t+\|v\|_{L_{\infty}(0, \infty ; V)} \sqrt{\tau_{0}+\delta_{0}}\left\{\lim _{k \rightarrow \infty} \int_{\tilde{t}_{k}}^{\tilde{t}_{k}+\tau_{0}+\delta_{0}}\|e(t)\|^{2} d t\right\}^{\frac{1}{2}}\right) \\
& \left.+\alpha_{1}^{2}\left(\lim _{k \rightarrow \infty} \int_{\tilde{t}_{k}}^{\tilde{t}_{k}+\tau_{0}+\delta_{0}} \| e\right\}^{\frac{1}{2}}+\delta_{0}\|v(t)\|_{L_{\infty}(0, \infty ; V)}\right) \\
& \times\left(\sqrt{\delta_{0}}\left\{\lim _{k \rightarrow \infty} \int_{\tilde{t}_{k}}^{\tilde{t}_{k}+\delta_{0}}\|e(t)\|^{2} d t\right\}^{2}\right. \\
= & 0,
\end{aligned}
$$

which is contradiction, and the theorem is proved. $\square$

We note that the persistence of excitation condition defined in Definition 3.5 is, in practice, difficult, if not impossible, to verify. However, this condition is analogous to a similar condition used to establish parameter convergence in an on-line identification scheme developed in [6]. In [8] a careful study and analysis of the persistence of excitation condition was carried out yielding valuable insight into how to recognize (based upon its performance) whether an adaptive scheme such as the one we treat here is either over-damped (i.e. the operator $-A_{0}$ is too stable) or under-damped (i.e. the persistency of excitation is insufficient). This information can then be used to tune the scheme (i.e. tune the reference model and reference input signal, $g$ ) so as to 
achieve a balance between the tracking error convergence (i.e. $\lim _{t \rightarrow \infty}|e(t)|=0$ ) and parameter convergence (i.e. $\lim _{t \rightarrow \infty}|q(t)-\bar{q}|_{Q}=\lim _{t \rightarrow \infty}|r(t)|_{Q}=0$ ). We note also that it is possible to establish a weaker parameter convergence result in either the absence of persistence of excitation, or the presence of partial persistence of excitation. The result and its proof are analogous to the corresponding notions in the case of a strict identification scheme (see [6] and [8]).

\section{Finite Dimensional Approximation}

The reference model, (2.7), (2.8), and the estimator, or adaptation law for $q,(2.25),(2.26)$, reside in the memory of a computer. Moreover, they are both, in general, infinite dimensional evolution equations. Consequently their real-time, or on-line, integration requires some form of finite dimensional approximation. This results in an approximating closed loop system. In this section we consider the finite dimensional approximation of the reference model and the adaptation law, and establish well-posedness and convergence results for the resulting approximating closed loop systems.

For each $n=1,2, \ldots$, let $H^{n}$ be a finite dimensional subspace of $H$ with $H^{n} \subset V$, and let $Q^{n}$ be a finite dimensional subspace of $Q$. Let $P^{n}: H \rightarrow H^{n}$ denote the orthogonal (with respect to the standard inner product on $H$ ) projection of $H$ onto $H^{n}$. We then use a Galerkin approach to approximate $(2.7),(2.8)$ and $(2.25),(2.26)$. For each $n=1,2, \ldots$, we consider the approximating reference model,

$$
\begin{gathered}
\left\langle D_{t} v^{n}(t), \varphi^{n}\right\rangle+\left\langle A_{0} v^{n}(t), \varphi^{n}\right\rangle=\left\langle g(t), \varphi^{n}\right\rangle, \quad \varphi^{n} \in H^{n}, \text { a.e. } t>0, \\
v^{n}(0)=v_{0}^{n},
\end{gathered}
$$

where $v^{n}(t), v_{0}^{n} \in H^{n}$, and the approximating adaptation law,

$$
\begin{gathered}
\left\langle D_{t} q^{n}(t), p^{n}\right\rangle_{Q}+\left\langle A_{1}\left(p^{n}\right) u(t), u(t)-v^{n}(t)\right\rangle=0, \quad p^{n} \in Q^{n}, \text { a.e. } t>0, \\
q^{n}(0)=q_{0}^{n},
\end{gathered}
$$

where $q^{n}(t), q_{0}^{n} \in Q^{n}$. Recalling the definition of the adaptive control law given in (2.23) or (2.24), for each $n=1,2, \ldots$, we define an approximating adaptive feedback control law, $f^{n}$, by

$$
f^{n}(t)=A\left(q^{n}(t)\right) u(t)-A_{0} u(t)+g(t), \quad \text { a.e. } t>0,
$$

or

$$
\left\langle f^{n}(t), \varphi\right\rangle=\left\langle A\left(q^{n}(t)\right) u(t), \varphi\right\rangle-\left\langle A_{0} u(t), \varphi\right\rangle+\langle g(t), \varphi\rangle, \quad \varphi \in V, \text { a.e. } t>0,
$$

where $q^{n}$ is determined by the system (4.1)-(4.4). Combining (4.1)-(4.4) and either (4.5) or (4.6) together with the plant, (2.3), (2.4), or (2.5), (2.6), we obtain what we will refer to as the approximating closed loop system

$$
\begin{gathered}
\left\langle D_{t} u^{n}(t), \varphi\right\rangle+\left\langle A_{0} u^{n}(t), \varphi\right\rangle+\left\langle A_{1}\left(\bar{q}-q^{n}(t)\right) u^{n}(t), \varphi\right\rangle=\langle g(t), \varphi\rangle, \quad \varphi \in V, \text { a.e. } t>0, \\
\left\langle D_{t} v^{n}(t), \varphi^{n}\right\rangle+\left\langle A_{0} v^{n}(t), \varphi^{n}\right\rangle=\left\langle g(t), \varphi^{n}\right\rangle, \quad \varphi^{n} \in H^{n} \text {, a.e. } t>0, \\
\left\langle D_{t} q^{n}(t), p^{n}\right\rangle_{Q}+\left\langle A_{1}\left(p^{n}\right) u^{n}(t), u^{n}(t)-v^{n}(t)\right\rangle=0, \quad p^{n} \in Q^{n} \text {, a.e. } t>0, \\
u^{n}(0)=u_{0}, \quad v^{n}(0)=v_{0}^{n}, \quad q^{n}(0)=q_{0}^{n} .
\end{gathered}
$$

We begin by establishing a well-posedness result for the system (4.7)-(4.10). Our approach is similar to the one taken earlier in Section 2 when we considered the well posedness of the closed 
loop system (2.27)-(2.30). We assume that Assumptions (A1)-(A9) are satisfied, and we first note that the equation for the reference model, (4.8), can be solved independently of equations (4.7) and (4.9). The solution, $v^{n} \in C([0, \infty) ; H) \cap C^{1}((0, \infty) ; H)$, is given by (see, for example, $[19])$

$$
v^{n}(t)=T_{0}^{n}(t) v_{0}^{n}+\int_{0}^{t} T_{0}^{n}(t-s) P^{n} g(s) d s, \quad t \geq 0,
$$

$\left\{T_{0}^{n}(t): t \geq 0\right\}$ is the uniformly exponentially stable analytic semigroup of bounded linear operators on $H^{n}$ generated by the Galerkin approximation, $-A_{0}^{n} \in \mathcal{L}\left(H^{n}, H^{n}\right)$, to the operator $-A_{0}$. That is, for each $n=1,2, \ldots, A_{0}^{n} \in \mathcal{L}\left(H^{n}, H^{n}\right)$ is the operator defined by $A_{0}^{n} \varphi^{n}=\psi^{n}$, for $\varphi^{n} \in H^{n}$, where $\psi^{n} \in H^{n}$ is the unique element in $H^{n}$ satisfying

$$
\left\langle A_{0} \varphi^{n}, \chi^{n}\right\rangle=\left\langle\psi^{n}, \chi^{n}\right\rangle, \quad \chi^{n} \in H^{n},
$$

guaranteed to exist by the Riesz Representation Theorem. Since $H^{n}$ was assumed to be finite dimensional, we have that

$$
T_{0}^{n}(t)=\exp \left(-A_{0}^{n} t\right)=e^{-A_{0}^{n} t}, \quad t \geq 0 .
$$

Let $\hat{X}=H \times Q$ be endowed with the inner product

$$
\langle(\varphi, q),(\psi, p)\rangle_{\hat{X}}=\langle\varphi, \psi\rangle+\langle q, p\rangle_{Q}, \quad(\varphi, q),(\psi, p) \in \hat{X}
$$

and let $|\cdot|_{\hat{X}}$ denote the corresponding induced norm. It follows that $\left\{\hat{X},\langle\cdot, \cdot\rangle_{\hat{X}},|\cdot|_{\hat{X}}\right\}$ is a Hilbert space. Moreover, as was done in the proof of Theorem 2.3, for the $\alpha \in(0,1)$ in Assumption (A6), define the Banach space $\left\{\hat{X}_{\alpha},|\cdot|_{\hat{X}_{\alpha}}\right\}$ by $\hat{X}_{\alpha}=H_{\alpha} \times Q$ with $|(\varphi, q)|_{\hat{X}_{\alpha}}=|\varphi|_{\alpha}+|q|_{Q}$, for $(\varphi, q) \in \hat{X}_{\alpha}$.

For $\lambda>0$ and $\psi \in C([0, \infty) ; H)$ define the mapping $\hat{G}_{\lambda}(\cdot, \cdot ; \psi):[0, \infty) \times \hat{X}_{\alpha} \rightarrow \hat{X}$ by

$$
\hat{G}_{\lambda}(t,(\varphi, q) ; \psi)=\left(g(t)-B(\varphi)\{\bar{q}-q\}, \lambda q-B(\varphi)^{\prime}\{\varphi-\psi(t)\}\right), \quad t \geq 0,(\varphi, q) \in \hat{X}_{\alpha},
$$

where for $\varphi \in V$ the operators $B(\varphi) \in \mathcal{L}\left(Q, V^{*}\right)$ and its Banach space adjoint $B(\varphi)^{\prime} \in \mathcal{L}(V, Q)$ are defined in (2.37) and (2.38), respectively. We note that since in the above definition, since $\varphi \in H_{\alpha}$. Assumption (A6) implies that the operator $B(\varphi)$ in fact has range in $H$, and that the operator $B(\varphi)^{\prime}$ is well defined on $H$. Consequently the mapping $\hat{G}_{\lambda}(\cdot, \cdot ; \psi)$ given by $(4.12)$ above is well defined on $[0, \infty) \times \hat{X}_{\alpha}$ with range in $\hat{X}$.

For $\lambda>0$, define the operator $\hat{A}_{\lambda}: \operatorname{Dom}\left(\hat{A}_{\lambda}\right) \subset \hat{X} \rightarrow \hat{X}$ by

$$
\begin{gathered}
\operatorname{Dom}\left(\hat{A}_{\lambda}\right)=D_{0} \times Q \\
\hat{A}_{\lambda}(\varphi, q)=\left(A_{0} \varphi, \lambda q\right), \quad(\varphi, q) \in \operatorname{Dom}\left(\hat{A}_{\lambda}\right) .
\end{gathered}
$$

The operator $-\hat{A}_{\lambda}$ is the infinitesimal generator of a uniformly exponentially stable analytic semigroup, $\left\{\hat{T}_{\lambda}(t): t \geq 0\right\}$, on $\hat{X}$, and $0 \in \rho\left(-\hat{A}_{\lambda}\right)$.

For $n=1,2, \ldots$, let $\hat{X}^{n}=H \times Q^{n}$, and let $P_{Q}^{n}: Q \rightarrow Q^{n}$ denote the orthogonal (with respect to the standard inner product on $\left.Q,\langle\cdot, \cdot\rangle_{Q}\right)$ projection of $Q$ onto $Q^{n}$. For $n=1,2, \ldots, \lambda>0$ and $\psi \in C([0, \infty) ; H)$ define the mapping $\hat{G}_{\lambda}^{n}(\cdot, \cdot ; \psi):[0, \infty) \times \hat{X}_{\alpha} \rightarrow \hat{X}^{n}$ by

$$
\hat{G}_{\lambda}^{n}(t,(\varphi, q) ; \psi)=\left(g(t)-B(\varphi)\{\bar{q}-q\}, \lambda P_{Q}^{n} q-P_{Q}^{n} B(\varphi)^{\prime}\{\varphi-\psi(t)\}\right), t \geq 0,(\varphi, q) \in \hat{X}_{\alpha} .
$$

For $n=1,2, \ldots$ and $t \geq 0$, let $\hat{x}^{n}(t)=\left(u^{n}(t), q^{n}(t)\right)$ and consider the system (4.7), (4.9) and (4.10) written in the form of an initial value problem in $\hat{X}^{n}$ as

$$
D_{t} \hat{x}^{n}(t)+\hat{A}_{\lambda} \hat{x}^{n}(t)=\hat{G}_{\lambda}^{n}\left(t, \hat{x}^{n}(t) ; v^{n}\right), \quad \text { a.e. } t>0,
$$




$$
\hat{x}^{n}(0)=\hat{x}_{0}^{n},
$$

where $\lambda>0, \hat{G}_{\lambda}^{n}$ is given by (4.15), $\hat{A}_{\lambda}$ is given by (4.13) and (4.14), $v^{n}$ is given by (4.11), and $\hat{x}_{0}^{n}=\left(u_{0}, q_{0}^{n}\right) \in \hat{X}^{n}$.

In Theorem 4.1 to follow, we establish that the initial value problem, (4.16), (4.17), has a unique local strong solution.

Theorem 4.1 If $u_{0} \in \operatorname{Dom}\left(A_{0}^{\alpha}\right)$, then for each $n=1,2, \ldots$, there exists a $T>0$ and a unique function $\hat{x}^{n} \in C([0, T) ; \hat{X}) \cap C^{1}((0, T) ; \hat{X})$ satisfying (4.16) and (4.17). Moreover, $\hat{x}^{n}$ satisfies the integral equation

$$
\hat{x}^{n}(t)=\hat{T}_{\lambda}(t) \hat{x}_{0}^{n}+\int_{0}^{t} \hat{T}_{\lambda}(t-s) \hat{G}_{\lambda}^{n}\left(s, \hat{x}^{n}(s) ; v^{n}\right) d s, \quad 0 \leq t<T .
$$

Proof. For each $n=1,2, \ldots$, let $\hat{X}_{\alpha}^{n}=\operatorname{Dom}\left(A_{0}^{\alpha}\right) \times Q^{n}$ be considered as a subspace of $\hat{X}_{\alpha}$, and let $\hat{U}^{n} \subset \hat{X}_{\alpha}^{n}$ be the neighborhood of $\hat{x}_{0}^{n}$ given by $\hat{U}^{n}=\left\{\hat{x}^{n} \in \hat{X}_{\alpha}^{n}:\left|\hat{x}^{n}-\hat{x}_{0}^{n}\right|_{\hat{X}_{\alpha}}<\varepsilon\right\}$. Let $\bar{T}>0$ and $\lambda>0$ be fixed. We show that there exists a constant $\hat{L}^{n}=\hat{L}^{n}\left(\varepsilon, \hat{x}_{0}^{n}, \lambda, \bar{T}\right)>0$, such that

$$
\left|\hat{G}_{\lambda}^{n}\left(t, \hat{\Phi}^{n} ; v^{n}\right)-\hat{G}_{\lambda}^{n}\left(s, \hat{\Psi}^{n} ; v^{n}\right)\right|_{\hat{X}} \leq \hat{L}^{n}\left\{|t-s|^{\nu}+|\varphi-\psi|_{\alpha}+\left|q^{n}-p^{n}\right|_{Q}\right\},
$$

for $0 \leq t, s \leq \bar{T}$, and $\hat{\Phi}^{n}=\left(\varphi, q^{n}\right), \hat{\Psi}^{n}=\left(\psi, p^{n}\right) \in \hat{U}^{n}$. The desired result will then follow as in the proof of Theorem 6.3.1 in [32].

Let $0 \leq t, s \leq \bar{T}$, and let $\hat{\Phi}^{n}=\left(\varphi, q^{n}\right), \hat{\Psi}^{n}=\left(\psi, p^{n}\right) \in \hat{U}^{n}$. Then

$$
\begin{aligned}
& \left|\hat{G}_{\lambda}^{n}\left(t, \hat{\Phi}^{n} ; v^{n}\right)-\hat{G}_{\lambda}^{n}\left(s, \hat{\Psi}^{n} ; v^{n}\right)\right|_{\hat{X}}^{2} \\
& \quad \leq 2|g(t)-g(s)|^{2}+2\left|B(\varphi)\left\{\bar{q}-q^{n}\right\}-B(\psi)\left\{\bar{q}-p^{n}\right\}\right|^{2} \\
& \quad+2 \lambda^{2}\left|q^{n}-p^{n}\right|_{Q}^{2}+2\left|B(\varphi)^{\prime}\left\{\varphi-v^{n}(t)\right\}-B(\psi)^{\prime}\left\{\psi-v^{n}(s)\right\}\right|_{Q}^{2} .
\end{aligned}
$$

Now Assumption (A9) implies that

$$
|g(t)-g(s)| \leq \delta|t-s|^{\nu}, \quad t, s \geq 0 .
$$

Assumptions ( $A 7)$ and ( $A 8)$ imply that

$$
\begin{aligned}
& \left|B(\varphi)\left\{\bar{q}-q^{n}\right\}-B(\psi)\left\{\bar{q}-p^{n}\right\}\right| \\
& \quad \leq|B(\varphi) \bar{q}-B(\psi) \bar{q}|+\left|B(\varphi) q^{n}-B(\varphi) p^{n}\right|+\left|B(\varphi) p^{n}-B(\psi) p^{n}\right| \\
& \quad \leq \gamma_{1}|\bar{q}|_{Q}|\varphi-\psi|_{\alpha}+\beta_{1}|\varphi|_{\alpha}\left|q^{n}-p^{n}\right|_{Q}+\gamma_{1}\left|p^{n}\right|_{Q}|\varphi-\psi|_{\alpha} .
\end{aligned}
$$

Now

$$
\begin{aligned}
& \left|B(\varphi)^{\prime}\left\{\varphi-v^{n}(t)\right\}-B(\psi)^{\prime}\left\{\psi-v^{n}(s)\right\}\right|_{Q} \\
& \quad \leq \sup _{|q|_{Q} \leq 1}\left|\left\langle B(\varphi)^{\prime} \varphi-B(\psi)^{\prime} \psi, q\right\rangle_{Q}\right|+\sup _{|q|_{Q} \leq 1}\left|\left\langle B(\varphi)^{\prime} v^{n}(t)-B(\psi)^{\prime} v^{n}(s), q\right\rangle_{Q}\right| .
\end{aligned}
$$

Assumptions ( $A 7)$ and ( $A 8)$ imply that

$$
\begin{aligned}
& \left|\left\langle B(\varphi)^{\prime} \varphi-B(\psi)^{\prime} \psi, q\right\rangle_{Q}\right| \\
& \quad \leq\left|\left\langle B(\varphi)^{\prime} \varphi-B(\varphi)^{\prime} \psi, q\right\rangle_{Q}\right|+\left|\left\langle B(\varphi)^{\prime} \psi-B(\psi)^{\prime} \psi, q\right\rangle_{Q}\right| \\
& \quad \leq \kappa_{\alpha}\left\{\beta_{1}|\varphi|_{\alpha}+\gamma_{1}|\psi|_{\alpha}\right\}|\varphi-\psi|_{\alpha}|q|_{Q},
\end{aligned}
$$


where, recalling that the space $\left\{H_{\alpha},|\cdot|_{\alpha}\right\}$ is densely and continuously embedded in $H, \kappa_{\alpha}$ is such that $|\xi| \leq \kappa_{\alpha}|\xi|_{\alpha}$, for $\xi \in H_{\alpha}$. Assumptions (A7) and (A8) also imply that

$$
\begin{aligned}
& \left|\left\langle B(\varphi)^{\prime} v^{n}(t)-B(\psi)^{\prime} v^{n}(s), q\right\rangle_{Q}\right| \\
& \quad \leq\left|\left\langle B(\varphi)^{\prime} v^{n}(t)-B(\varphi)^{\prime} v^{n}(s), q\right\rangle_{Q}\right|+\left|\left\langle B(\varphi)^{\prime} v^{n}(s)-B(\psi)^{\prime} v^{n}(s), q\right\rangle_{Q}\right| \\
& \quad \leq \beta_{1}|\varphi|_{\alpha}\left|v^{n}(t)-v^{n}(s)\right||q|_{Q}+\gamma_{1}\left|v^{n}(s)\right||\varphi-\psi|_{\alpha}|q|_{Q} \\
& \quad \leq \kappa_{1}|\varphi|_{\alpha}|t-s|^{\nu}|q|_{Q}+\kappa_{2}|\varphi-\psi|_{\alpha}|q|_{Q},
\end{aligned}
$$

for some positive constants $\kappa_{1}$ and $\kappa_{2}$, where in the final estimate in (4.25) above, we have applied a regularity result for mild solutions to systems governed by analytic semigroups given in Theorem 4.3.1 in [32], and the fact that $v^{n} \in C([0, \bar{T}] ; H)$ and is therefore $H$-bounded uniformly on $[0, \bar{T}]$.

Combining (4.20)-(4.25), we obtain (4.19), and the theorem is proved.

It is also possible to establish a global existence result for the solution to the system (4.16), (4.17). However to do this we require the following additional assumption.

(A12) The operator $A_{1}(\bar{q}): V \rightarrow V^{*}$ is monotone in the sense that

$$
\left\langle A_{1}(\bar{q}) \varphi-A_{1}(\bar{q}) \psi, \varphi-\psi\right\rangle \geq 0, \quad \varphi, \psi \in V .
$$

We note that Assumption (A12) is not excessively restrictive in that monotonicity can be demonstrated for relatively large classes of nonlinear operators. It corresponds physically to some form of energy dissipation in the plant. In particular, we note that the operator $A_{1}(\bar{q})$ appearing in the example presented in Section 2.1 satisfies Assumption (A12) (see (2.62)).

Theorem 4.2 Suppose that Assumptions (A1)-(A9) and (A12) hold and that $u_{0} \in \operatorname{Dom}\left(A_{0}^{\alpha}\right)$. Then for each $n=1,2, \ldots$, the initial value problem (4.16),(4.17) has a unique solution $\hat{x}^{n}=$ $\left(u^{n}, q^{n}\right)$ which exists for all $t \geq 0$.

Proof. As in the proof of Theorem 2.5, we show that for each $n=1,2, \ldots$, the local solution, $\hat{x}^{n}$ to the initial value problem (4.16), (4.17) shown to exist in Theorem 4.1 can be continued by arguing that $\left|\hat{x}^{n}(t)\right|_{\hat{X}_{\alpha}}$ remains bounded as $t \uparrow T$. For $t \in[0, T)$ we have

$$
\left|\hat{x}^{n}(t)\right|_{\hat{X}_{\alpha}}=\left|u^{n}(t)\right|_{\alpha}+\left|q^{n}(t)\right|_{Q}
$$

We begin by determining a bound for $\left|q^{n}(t)\right|_{Q}$ as $t \uparrow T$. From (4.7) and (4.9), and Assumptions $(A 1),(A 2),(A 4)$, and $(A 12)$, for $t \in(0, T)$, and $\theta$ the zero vector in $V$, we obtain the estimate

$$
\begin{aligned}
& \frac{1}{2}\left\{D_{t}\left|u^{n}(t)\right|^{2}+D_{t}\left|q^{n}(t)\right|_{Q}^{2}\right\} \\
& =\left\langle D_{t} u^{n}(t), u^{n}(t)\right\rangle+\left\langle D_{t} q^{n}(t), q^{n}(t)\right\rangle_{Q} \\
& =-\left\langle A_{0} u^{n}(t), u^{n}(t)\right\rangle-\left\langle A_{1}(\bar{q}) u^{n}(t), u^{n}(t)\right\rangle+\left\langle g(t), u^{n}(t)\right\rangle+\left\langle A_{1}\left(q^{n}(t)\right) u^{n}(t), v^{n}(t)\right\rangle \\
& =-\left\langle A_{0} u^{n}(t), u^{n}(t)\right\rangle-\left\langle A_{1}(\bar{q}) u^{n}(t)-A_{1}(\bar{q}) \theta, u^{n}(t)\right\rangle-\left\langle A_{1}(\bar{q}) \theta, u^{n}(t)\right\rangle \\
& \quad \quad+\left\langle g(t), u^{n}(t)\right\rangle+\left\langle A_{1}\left(q^{n}(t)\right) u^{n}(t), v^{n}(t)\right\rangle \\
& \leq-\left\langle A_{0} u^{n}(t), u^{n}(t)\right\rangle+\left|\left\langle A_{1}(\bar{q}) \theta, u^{n}(t)\right\rangle\right|+\left\langle g(t), u^{n}(t)\right\rangle+\left\langle A_{1}\left(q^{n}(t)\right) u^{n}(t), v^{n}(t)\right\rangle \\
& \leq-\rho_{0}\left\|u^{n}(t)\right\|^{2}+\|g(t)\|_{*}\left|u^{n}(t)\left\|+\left.\alpha_{1}\left|q^{n}(t)\right|\right|_{Q}\right\| u^{n}(t)\|\| v^{n}(t)\right| .
\end{aligned}
$$


Now $v^{n} \in C([0, T] ; H)$. But $v^{n}(t) \in H^{n}, t \in[0, T]$, and $H^{n} \subset V$ finite dimensional imply that $v^{n} \in C([0, T] ; V)$, and therefore that $\left\|v^{n}(t)\right\|$ is bounded for $t \in[0, T]$. Consequently, for $\varepsilon>0$, (4.26) yields

$$
\begin{aligned}
& \frac{1}{2}\left\{D_{t}\left|u^{n}(t)\right|^{2}+D_{t}\left|q^{n}(t)\right|_{Q}^{2}\right\} \\
& \quad \leq-\rho_{0}\left\|\left.u^{n}(t)\right|^{2}+\frac{1}{2 \varepsilon}\right\| g(t)\left\|_{*}^{2}+\frac{\varepsilon}{2}\right\| u^{n}(t)\left\|^{2}+\frac{\kappa^{n}}{2 \varepsilon}\left|q^{n}(t)\right|_{Q}^{2}+\frac{\kappa^{n} \varepsilon}{2}\right\| u^{n}(t) \|^{2},
\end{aligned}
$$

or

$$
\frac{1}{2}\left\{D_{t}\left|u^{n}(t)\right|^{2}+D_{t}\left|q^{n}(t)\right|_{Q}^{2}\right\}+\left\{\rho_{0}-\left(1+\kappa^{n}\right) \frac{\varepsilon}{2}\right\}\left\|u^{n}(t)\right\|^{2} \leq \frac{1}{2 \varepsilon} \|\left. g(t)\right|_{*} ^{2}+\frac{\kappa^{n}}{2 \varepsilon}\left|q^{n}(t)\right|_{Q}^{2},
$$

for some $\kappa^{n}>0$. Choosing $\varepsilon=\varepsilon^{n}=2 \rho_{0} /\left(1+\kappa^{n}\right)$, we obtain

$$
D_{t}\left|u^{n}(t)\right|^{2}+D_{t}\left|q^{n}(t)\right|_{Q}^{2} \leq \mu_{1}|| g(t) \|_{*}^{2}+\mu_{2}^{n}\left|q^{n}(t)\right|_{Q}^{2}, \quad 0 \leq t<T
$$

for some $\mu_{1}, \mu_{2}^{n}>0$. Integrating both sides of the estimate in (4.27) from 0 to $t$, we find that for $0 \leq t<T$

$$
\begin{aligned}
& \left|u^{n}(t)\right|^{2}+\left|q^{n}(t)\right|_{Q}^{2} \\
& \quad \leq\left|u_{0}\right|^{2}+\left|q_{0}^{n}\right|_{Q}^{2}+\mu_{1} \int_{0}^{t}\|g(s)\|_{*}^{2} d s+\mu_{2}^{n} \int_{0}^{t}\left|q^{n}(s)\right|_{Q}^{2} d s .
\end{aligned}
$$

Applying the generalized Gronwall inequality (see, for example, [14]) to (4.28) above, we obtain

$$
\begin{aligned}
\left|u^{n}(t)\right|^{2}+\left|q^{n}(t)\right|_{Q}^{2} & \leq \zeta^{n}(t)+\mu_{2}^{n} \int_{0}^{t} \epsilon^{\mu_{2}^{n}(t-s)} \zeta^{n}(s) d s . \\
& \leq\left(1+\mu_{2}^{n} T \epsilon^{\mu_{2}^{n} T}\right) \zeta^{n}(T) \\
& =\kappa_{T}^{n}, \quad 0 \leq t<T
\end{aligned}
$$

where for each $n=1,2, \ldots, \zeta^{n}$ is the monotone increasing function on $[0, T]$ given by

$$
\zeta^{n}(t)=\left|u_{0}\right|^{2}+\left|q_{0}^{n}\right|_{Q}^{2}+\left.\mu_{1} \int_{0}^{t}|| g(s)\right|_{*} ^{2} d s, \quad 0 \leq t \leq T .
$$

Now for $t \in[0, T)$, from (4.18) we obtain

$$
A_{0}^{\alpha} u^{n}(t)=A_{0}^{\alpha} T_{0}(t) u_{0}+\int_{0}^{t} A_{0}^{\alpha} T_{0}(t-s)\left\{g(s)-B\left(u^{n}(s)\right)\left\{\bar{q}-q^{n}(s)\right\}\right\} d s .
$$

It follows from (4.30) and Assumptions ( $A 4)$ and $(A 7)$, that for $t \in[0, T)$, we have

$$
\begin{aligned}
& \left|u^{n}(t)\right|_{\alpha} \\
& \quad \leq e^{-\rho_{0} K^{-2} t}\left|u_{0}\right|_{\alpha}+\int_{0}^{t} M_{\alpha}(t-s)^{-\alpha} e^{-\rho_{0} K^{-2}(t-s)}\left\{|g(s)|+\beta_{1}\left\{|\bar{q}|_{Q}+\left|q^{n}(s)\right|_{Q}\right\}\left|u^{n}(s)\right|_{\alpha}\right\} d s,
\end{aligned}
$$

for some positive constant $M_{\alpha}$ (see [32]). Assumption (A9) and (4.29) then imply that

$$
\begin{aligned}
& \left|u^{n}(t)\right|_{\alpha} \\
& \quad \leq\left|u_{0}\right|_{\alpha}+M_{\alpha}\|g\|_{C([0, T] ; H)} \frac{T^{1-\alpha}}{1-\alpha}+M_{\alpha} \beta_{1}\left\{|\bar{q}|_{Q}+\sqrt{\kappa_{T}^{n}}\right\} \int_{0}^{t}(t-s)^{-\alpha}\left|u^{n}(s)\right|_{\alpha} d s,
\end{aligned}
$$


for $0 \leq t<T$. An application of Lemma 5.6.7 in [32] to the estimate given in (4.31) above, then yields the existence of a constant, $\lambda_{T}^{n}>0$, for which

$$
\left|u^{n}(t)\right|_{\alpha} \leq \lambda_{T}^{n}, \quad 0 \leq t<T .
$$

Combining (4.29) and (4.32), we obtain the desired result, and the theorem is proved. $\square$

Before we present our convergence result, we discuss some computational considerations, and in particular, the matrix representations for the finite dimensional approximating reference model, (4.1), (4.2), and adaptation law, (4.3), (4.4). For each $n=1,2, \ldots$ let $\left\{\varphi_{j}^{n}\right\}_{j=1}^{k^{n}}$ be a basis for $H^{n}$ and let $\left\{p_{j}^{n}\right\}_{j=1}^{\ell^{n}}$ be a basis for $Q^{n}$. Let

$$
\begin{aligned}
& v^{n}(t)=\sum_{j=1}^{k^{n}} \tilde{v}_{j}^{n}(t) \varphi_{j}^{n}, \quad t \geq 0, \\
& q^{n}(t)=\sum_{j=1}^{\ell^{n}} \tilde{q}_{j}^{n}(t) p_{j}^{n}, \quad t \geq 0 .
\end{aligned}
$$

That is, for each $t \geq 0$, let $\tilde{v}^{n}(t) \in \mathbf{R}^{k^{n}}$ and $\tilde{q}^{n}(t) \in \mathbf{R}^{\ell^{n}}$ be, respectively, the vector representations for $v^{n}(t) \in H^{n}$ and $q^{n} \in Q^{n}$ with respect to the bases $\left\{\varphi_{j}^{n}\right\}_{j=1}^{k^{n}}$ and $\left\{p_{j}^{n}\right\}_{j=1}^{\ell^{n}}$. We choose $v_{0}^{n}=P^{n} v_{0} \in H^{n}$ and $q_{0}^{n}=P_{Q}^{n} q_{0} \in Q^{n}$.

The matrix form of the approximating reference model, (4.1), (4.2), then becomes

$$
\begin{aligned}
M^{n} D_{t} \tilde{v}^{n}(t)+K^{n} \tilde{v}^{n}(t) & =g^{n}(t), \quad t>0, \\
M^{n} \tilde{v}^{n}(0) & =\tilde{v}_{0}^{n},
\end{aligned}
$$

where the $k^{n} \times k^{n}$ matrices $M^{n}$ and $K^{n}$ are given by

$$
\left[M^{n}\right]_{i, j}=\left\langle\varphi_{j}^{n}, \varphi_{i}^{n}\right\rangle \quad \text { and } \quad\left[K^{n}\right]_{i, j}=\left\langle A_{0} \varphi_{j}^{n}, \varphi_{i}^{n}\right\rangle \quad i, j=1,2, \ldots, k^{n},
$$

respectively, and

$$
\left[\tilde{v}_{0}^{n}\right]_{i}=\left\langle v_{0}, \varphi_{i}^{n}\right\rangle \quad \text { and } \quad\left[g^{n}(t)\right]_{i}=\left\langle g(t), \varphi_{i}^{n}\right\rangle \quad i=1,2, \ldots, k^{n}, t \geq 0 .
$$

Note that since $\left\{\varphi_{j}^{n}\right\}_{j=1}^{k^{n}}$ is a basis for $H^{n}$, the matrix $M^{n}$ is nonsingular.

For $u(t) \in V$, the output of the plant, (2.3). (2.4), or (2.5), (2.6), at time $t \geq 0$, the matrix form of the approximating adaptation law, (4.3), (4.4), is given by

$$
\begin{aligned}
M_{Q}^{n} D_{t} \tilde{q}^{n}(t)-L^{n}(u(t)) \tilde{v}^{n}(t) & =-h^{n}(u(t)), \quad t>0, \\
M_{Q}^{n} \tilde{q}^{n}(0) & =\tilde{q}_{0}^{n},
\end{aligned}
$$

where the $\ell^{n} \times \ell^{n}$ matrix $M_{Q}^{n}$ is given by

$$
\left[M_{Q}^{n}\right]_{i, j}=\left\langle p_{j}^{n}, p_{i}^{n}\right\rangle_{Q}, \quad i, j=1,2, \ldots, \ell^{n},
$$

for $\varphi \in V$, the $\ell^{n} \times k^{n}$ matrix $L^{n}(\varphi)$ and the $\ell^{n}$-vector $h^{n}(\varphi)$ are given by

$$
\left[L^{n}(\varphi)\right]_{i, j}=\left\langle A_{1}\left(p_{i}^{n}\right) \varphi, \varphi_{j}^{n}\right\rangle \text { and }\left[h^{n}(\varphi)\right]_{i}=\left\langle A_{1}\left(p_{i}^{n}\right) \varphi, \varphi\right\rangle, \quad i=1,2, \ldots, \ell^{n}, j=1,2, \ldots, k^{n},
$$

respectively, and the $\ell^{n}$-vector $\tilde{q}_{0}^{n}$ is given by

$$
\left[\tilde{q}_{0}^{n}\right]_{i}=\left\langle q_{0}, p_{i}^{n}\right\rangle_{Q}, \quad i=1,2, \ldots, \ell^{n} .
$$


Once again, since $\left\{p_{j}^{n}\right\}_{j=1}^{\ell^{n}}$ is a basis for $Q^{n}$, the matrix $M_{Q}^{n}$ is nonsingular.

Combining (4.34), (4.35) and (4.36), (4.37), for $u(t) \in V$, the output of the plant, (2.3), (2.4), or (2.5), (2.6), at time $t \geq 0$, the $k^{n}+\ell^{n}$ dimensional linear system

$$
\begin{gathered}
{\left[\begin{array}{c}
D_{t} \tilde{v}^{n}(t) \\
D_{t} \tilde{q}^{n}(t)
\end{array}\right]+\left[\begin{array}{cc}
\left(M^{n}\right)^{-1} K^{n} & 0 \\
-\left(M_{Q}^{n}\right)^{-1} L^{n}(u(t)) & 0
\end{array}\right]\left[\begin{array}{c}
\tilde{v}^{n}(t) \\
\tilde{q}^{n}(t)
\end{array}\right]=\left[\begin{array}{c}
\left(M^{n}\right)^{-1} g^{n}(t) \\
-\left(M_{Q}^{n}\right)^{-1} h^{n}(u(t))
\end{array}\right] \quad t>0,} \\
{\left[\begin{array}{c}
\tilde{v}^{n}(0) \\
\tilde{q}^{n}(0)
\end{array}\right]=\left[\begin{array}{c}
\left(M^{n}\right)^{-1} \tilde{v}_{0}^{n} \\
\left(M_{Q}^{n}\right)^{-1} \tilde{q}_{0}^{n}
\end{array}\right]}
\end{gathered}
$$

must be integrated to determine the state of the approximating reference model, $v^{n}(t)$, and the approximating parameter estimator, $q^{n}(t)$, at time $t>0$. The estimate for the parameters is given by (4.33), and the control input is given by

$$
f^{n}(t)=A\left(\sum_{j=1}^{\ell^{n}} \tilde{q}_{j}^{n}(t) p_{j}^{n}\right) u(t)-A_{0} u(t)+g(t), \quad t \geq 0 .
$$

We are now ready to turn to our convergence result. We require the following rather standard assumptions on the approximation properties of the finite dimensional subspaces $H^{n}$ and $Q^{n}$.

(A13) The subspace $H^{n}$ is such that for each $n=1,2, \ldots$, there exists a mapping $\pi^{n} \in \mathcal{L}(V, V)$ for which $\pi^{n} \varphi \in H^{n}, \varphi \in V$, and

$$
\lim _{n \rightarrow \infty}\left\|\pi^{n} \varphi-\varphi\right\|=0, \quad \varphi \in V .
$$

(A14) The subspace $Q^{n}$ is such that

$$
\lim _{n \rightarrow \infty}\left|P_{Q}^{n} q-q\right|_{Q}=0, \quad q \in Q .
$$

We note that Assumption ( $A 13$ ) together with the dense and continuous embedding of $V$ in $H$ is sufficient to conclude that

$$
\lim _{n \rightarrow \infty}\left|P^{n} \varphi-\varphi\right|=0, \quad \varphi \in H .
$$

We note further that in many cases it is possible to choose $\pi_{n}=P^{n}$. Indeed, this is in fact the case for polynomial spline based subspaces. Assumption (A13) can then be verified using the estimates found in, for example, [35].

The following theorem concerning the convergence of the approximating semigroups $\left\{T_{0}^{n}(t)\right.$ : $t \geq 0\}$ to the semigroup $\left\{T_{0}(t): t \geq 0\right\}$ is established in [3] 11sing the well known Trotter-Kato theorem (see, for example, [19] and [32]).

Theorem 4.3 Under Assumptions (A3), (A4), and (A13), for each $T>0$ we have the following results.

(i) There exists a constant $M_{0}>0$, independent of $n$, for which

$$
\left\|T_{0}^{n}(t) \varphi^{n}\right\| \leq M_{0}\left\|\varphi^{n}\right\|, \quad \varphi^{n} \in H^{n} .
$$


(ii) For $\varphi \in H$ and $t \in[0, T]$

$$
\lim _{n \rightarrow \infty}\left|T_{0}^{n}(t) P^{n} \varphi-T_{0}(t) \varphi\right|=0,
$$

uniformly in $t$ for $t$ in compact subintervals of $[0, T]$.

(iii) For $\varphi \in V$ and $t \in[0, T]$

$$
\lim _{n \rightarrow \infty}\left\|T_{0}^{n}(t) \pi^{n} \varphi-T_{0}(t) \varphi\right\|=0,
$$

uniformly in $t$ for $t$ in compact subintervals of $[0, T]$.

(iv) For $\varphi \in H$ and $t \in(0, T]$

$$
\lim _{n \rightarrow \infty}\left\|T_{0}^{n}(t) P^{n} \varphi-T_{0}(t) \varphi\right\|=0,
$$

uniformly in $t$ for $t$ in compact subintervals of $(0, T]$.

Once $(i)$ has been established, the essence of the proof of (ii)-(iv) is demonstrating resolvent convergence in $V$. Let $\lambda>0$ and $\varphi \in V$, and set $\psi=\left(\lambda I+A_{0}\right)^{-1} \varphi$ and $\psi^{n}=\left(\lambda I+A_{0}^{n}\right)^{-1} \pi^{n} \varphi$, $n=1,2, \ldots$. The triangle inequality yields

$$
\left\|\psi-\psi^{n}\right\| \leq\left\|\psi-\pi^{n} \psi\right\|+\left\|\pi^{n} \psi-\psi^{n}\right\| .
$$

Assumption (A13) implies that the first term on the right hand side of the estimate in (4.38) tends to zero as $n \rightarrow \infty$. With regard to the second term, using Assumptions ( $A 3)$ and (A4) we obtain, for any $\varepsilon>0$,

$$
\begin{aligned}
& \rho_{0}\left\|\psi^{n}-\pi^{n} \psi\right\|^{2} \leq\left\langle A_{0}\left\{\psi^{n}-\pi^{n} \psi\right\}, \psi^{n}-\pi^{n} \psi\right\rangle \\
& =\left\langle\left(\lambda I+A_{0}^{n}\right) \psi^{n}-\left(\lambda I+A_{0}\right) \psi, \psi^{n}-\pi^{n} \psi\right\rangle+\lambda\left\langle\psi-\pi^{n} \psi, \psi^{n}-\pi^{n} \psi\right\rangle \\
& \quad-\lambda\left\langle\psi^{n}-\pi^{n} \psi, \psi^{n}-\pi^{n} \psi\right\rangle+\left\langle A_{0}\left\{\psi-\pi^{n} \psi\right\}, \psi^{n}-\pi^{n} \psi\right\rangle \\
& \leq K\left\|\varphi-\pi^{n} \varphi\right\|\left\|\psi^{n}-\pi^{n} \psi\right\|+\lambda\left\|\psi-\pi^{n} \psi\right\|\left\|\psi^{n}-\pi^{n} \psi\right\|-\lambda\left|\pi^{n} \psi-\psi^{n}\right|^{2} \\
& \quad+\alpha_{0}\left\|\psi-\pi^{n} \psi\right\|\left\|\psi^{n}-\pi^{n} \psi\right\| \\
& \leq \frac{K}{2 \varepsilon}\left\|\varphi-\pi^{n} \varphi\right\|^{2}+\frac{\lambda+\alpha_{0}}{2 \varepsilon}\left\|\psi-\pi^{n} \psi\right\|^{2}+\frac{1}{2}\left\{K+\lambda+\alpha_{0}\right\} \varepsilon\left\|\psi^{n}-\pi^{n} \psi\right\|^{2} .
\end{aligned}
$$

Choosing $\varepsilon>0$ sufficiently small, we find that

$$
\left\|\psi^{n}-\pi^{n} \psi\right\|^{2} \leq \nu_{1}\left\|\varphi-\pi^{n} \varphi\right\|^{2}+\nu_{2}\left\|\psi-\pi^{n} \psi\right\|^{2}
$$

for some constants $\nu_{1}, \nu_{2}>0$. Invoking Assumption (A13), and recalling (4.38), we obtain

$$
\lim _{n \rightarrow \infty}\left\|\left(\lambda I+A_{0}^{n}\right)^{-1} \pi^{n} \varphi-\left(\lambda I+A_{0}\right)^{-1} \varphi\right\|=0, \quad \varphi \in V .
$$

We will require the following corollary to Theorem 4.3 above.

Corollary 4.4 If $v_{0} \in V$ and $v_{0}^{n}=\pi^{n} v_{0}$, then under Assumptions (A3), (A4), (A9), and (A13), for each $T>0$ we have the following results.

(i) For each $t \in[0, T]$

$$
\lim _{n \rightarrow \infty} \| v^{n}(t)-v(t) \mid=0
$$


(ii) There exists a constant $\kappa_{v^{n}}=\kappa_{v^{n}}(T)>0$, independent of $n$ for which

$$
\left\|v^{n}(t)\right\| \leq \kappa_{v^{n}}, \quad 0 \leq t \leq T .
$$

Proof. From (2.11) and (4.11) for $t \in[0, T]$, we obtain

$$
\begin{aligned}
& \left\|v^{n}(t)-v(t)\right\| \\
& \quad \leq\left\|T_{0}^{n}(t) \pi^{n} v_{0}-T_{0}(t) v_{0}\right\|+\int_{0}^{t}\left\|T_{0}^{n}(t-s) P^{n} g(s)-T_{0}(t-s) g(s)\right\| d s .
\end{aligned}
$$

Statement (iii) of Theorem 4.3 implies that the first term on the right hand side of the above expression tends to zero as $n \rightarrow \infty$. Statement (iv) of Theorem $4.3 \mathrm{implies}$ that the term under the integral sign tends to zero for almost every $s \in[0, T]$. Moreover, Lemma 3.6.2 in [38] implies that

$$
\left\|T_{0}^{n}(t-s) P^{n} g(s)-T_{0}(t-s) g(s)\right\| \leq \frac{C}{(t-s)^{\frac{1}{2}}}|g(s)|, \quad 0 \leq s<t .
$$

It follows that an application of the Lebesgue Dominated Convergence Theorem then yields $(i)$.

Statement (ii) is established analogously. Indeed, for $t \in[0, T],(4.11)$ yields

$$
\begin{aligned}
\left\|v^{n}(t)\right\| & \leq\left\|T_{0}^{n}(t) \pi^{n} v_{0}\right\|+\int_{0}^{t}\left\|T_{0}^{n}(t-s) P^{n} g(s)\right\| d s \\
& \leq M_{0} \kappa_{\pi}\left\|v_{0}\right\|+\int_{0}^{t} \frac{C}{(t-s)^{\frac{1}{2}}}|g(s)| d s \\
& \leq M_{0} \kappa_{\pi}\left\|v_{0}\right\|+2 C\|g\|_{C([0, T] ; H)} \sqrt{T} \\
& =\kappa_{v^{n}}(T),
\end{aligned}
$$

where $\kappa_{\pi}>0$ is the uniform bound on the operators $\pi^{n} \in \mathcal{L}(V, V), n=1,2, \ldots$, guaranteed to exist by Assumption (A13) and the Uniform Boundedness Principle. This proves the theorem. $\square$

Using Corollary 4.4, the next corollary follows immediately by inspection.

Corollary 4.5 Suppose that Assumptions (A1)-(A9) and (A12)-(A14) hold, and that $u_{0} \in$ $\operatorname{Dom}\left(A_{0}^{\alpha}\right)$. Suppose further that $v_{0} \in V, v_{0}^{n}=\pi^{n} v_{0}$, and that $q_{0}^{n}=P_{Q}^{n} q_{0}$. Then the constants $\kappa_{T}^{n}$ and $\lambda_{T}^{n}$ defined in the proof of Theorem 4.2 above are in fact independent of $n$.

The implication of Corollary 4.5 is that for $T>0$ fixed, $\left|u^{n}(t)\right|_{\alpha}$ and $\left|q^{n}(t)\right|_{Q}$ are bounded uniformly in $n$ and $t$ for $t \in[0, T]$, where for each $n=1,2, \ldots u^{n}$ and $q^{n}$ satisfy (4.7)-(4.10). That is there exist constants $\kappa_{T}>0$ and $\lambda_{T}>0$, independent of $n$ for which

$$
\left|\hat{x}^{n}(t)\right|_{\hat{X}_{\alpha}}=\left|u^{n}(t)\right|_{\alpha}+\left|q^{n}(t)\right|_{Q} \leq \lambda_{T}+\sqrt{\kappa_{T}}, \quad 0 \leq t \leq T,
$$

where for each $n=1,2, \ldots \hat{x}^{n}$ is the solution to the initial value problem (4.16), (4.17).

Our convergence result is given in Theorem 4.7 below. Its proof requires the following lemma.

Lemma 4.6 If $\operatorname{Dom}\left(A_{0}^{\alpha}\right) \subset V$ for some $\alpha \in(0,1)$, then the Banach space $\left\{H_{\alpha},|\cdot|_{\alpha}\right\}$ defined by $H_{\alpha}=\operatorname{Dom}\left(A_{0}^{\alpha}\right)$ and $|\varphi|_{\alpha}=\left|A_{0}^{\alpha} \varphi\right|, \varphi \in \operatorname{Dom}\left(A_{0}^{\alpha}\right)$ is continuously embedded in $V$. That is, there exists a constant, $K_{V}>0$, for which

$$
\|\varphi\| \leq K_{V}|\varphi|_{\alpha}, \quad \varphi \in \operatorname{Dom}\left(A_{0}^{\alpha}\right) .
$$


Proof. It can be shown (see [27], page 11) that there exists a linear, self adjoint, and positive operator $\Lambda: \operatorname{Dom}(\Lambda) \subset H \rightarrow H$, for which $\operatorname{Dom}(\Lambda)=V$ and for which the norm $\|\cdot\|_{\Lambda}$ on $V$ given by

$$
\|\varphi\|_{\Lambda}=\left\{|\varphi|^{2}+|\Lambda \varphi|^{2}\right\}^{\frac{1}{2}}, \quad \varphi \in V
$$

is equivalent to the standard norm, $\|\cdot\|$, on $V$. Then, for $\varphi \in \operatorname{Dom}\left(A_{0}^{\alpha}\right)$, Corollary 2.6.11 in [32] implies that

$$
\|\varphi\|^{2} \leq K_{1}\left\{|\varphi|^{2}+|\Lambda \varphi|^{2}\right\} \leq K_{2}\left\{|\varphi|^{2}+\left|A_{0}^{\alpha} \varphi\right|^{2}\right\} \leq K_{2}\|\varphi\|_{\alpha}^{2} \leq K_{V}^{2}|\varphi|_{\alpha}^{2},
$$

for some constants $K_{1}, K_{2}, K_{V}>0$, where the norm $\|\cdot\|_{\alpha}$ on $H_{\alpha}$ was defined and discussed in the proof of Theorem 2.3.

Theorem 4.7 Suppose that Assumptions (A1)-(A9) and Assumptions (A12)-(A14) hold, that $u_{0} \in \operatorname{Dom}\left(A_{0}^{\alpha}\right), \alpha \in(0,1)$ as in Assumption $(A 6)$, and that $v_{0}^{n}=\pi^{n} v_{0}$ and $q_{0}^{n}=P_{Q}^{n} q_{0}$. Then

$$
\lim _{n \rightarrow \infty}\left|u^{n}(t)-u(t)\right|_{\alpha}=\lim _{n \rightarrow \infty}\left|A_{0}^{\alpha} u^{n}(t)-A_{0}^{\alpha} u(t)\right|=0,
$$

and

$$
\lim _{n \rightarrow \infty}\left|q^{n}(t)-q(t)\right|_{Q}=0,
$$

uniformly in $t$, for $t \in[0, T]$, where $u^{n}, q^{n}$ satisfy (4.7)-(4.10), and $u$ and $q$ satisfy $(2.27)-(2.30)$. Moreover, if, in addition, the operator $A_{2}$ satisfies a Lipschitz condition of the form

$$
\left\|A_{2} \varphi-A_{2} \psi\right\|_{*} \leq \gamma_{2}|\varphi-\psi|_{\alpha}, \quad \varphi, \psi \in \operatorname{Dom}\left(A_{0}^{\alpha}\right),
$$

for some $\gamma_{2}>0$, where $\alpha \in(0,1)$ is as in Assumption $(A 6)$, then we have

$$
\lim _{n \rightarrow \infty} f^{n}(t)=f(t)
$$

in $V^{*}$ uniformly in $t$, for $t \in[0, T]$, and therefore that

$$
\lim _{n \rightarrow \infty} f^{n}=f
$$

in $L_{2}\left(0, T ; V^{*}\right)$, for each $T>0$, where for each $n=1,2, \ldots, f^{n}$ is given by (4.5) or (4.6), and $f$ is given by $(2.23)$ or $(2.24)$.

Before we prove Theorem 4.7, we note that (4.40) implies that

$$
\lim _{n \rightarrow \infty}\left|u^{n}(t)-u(t)\right| \leq \lim _{n \rightarrow \infty}\left|u^{n}(t)-u(t)\right|_{\alpha} \leq \kappa_{\alpha} \lim _{n \rightarrow \infty}\left|u^{n}(t)-u(t)\right|_{\alpha}=0,
$$

uniformly in $t$ for $t \in[0, T]$. Moreover, (4.40) together with Lemma 4.6 also imply that

$$
\lim _{n \rightarrow \infty}\left\|u^{n}(t)-u(t)\right\| \leq K_{V} \lim _{n \rightarrow \infty}\left|u^{n}(t)-u(t)\right|_{\alpha}=0,
$$

uniformly in $t$ for $t \in[0, T]$.

Proof of Theorem 4.7. For each $t \geq 0$, let $\hat{x}(t)=(u(t), q(t))$, where $u$ and $q$ satisfy $(2.27)$ (2.30). Then for each $\lambda>0, \hat{x}$ satisfies

$$
\hat{x}(t)=\hat{T}_{\lambda}(t) \hat{x}_{0}+\int_{0}^{t} \hat{T}_{\lambda}(t-s) \hat{G}_{\lambda}(s, \hat{x}(s) ; v) d s, \quad t \geq 0,
$$


where $\hat{G}_{\lambda}$ is given by (4.12), $\left\{\hat{T}_{\lambda}(t): t \geq 0\right\}$ is the uniformly exponentially stable semigroup of bounded linear operators on $\hat{X}$ generated by the operator $-\hat{A}_{\lambda}$ defined in (4.13) and (4.14), and $v$ satisfies (2.7), (2.8) and is given by (2.11). Subtracting (4.45) from (4.18) and taking norms in $\hat{X}_{\alpha}$, for each $t \geq 0$, we find that

$$
\begin{aligned}
& \left|\hat{x}^{n}(t)-\hat{x}(t)\right|_{\hat{X}_{\alpha}} \\
& \leq e^{-\lambda t}\left|P_{Q}^{n} q_{0}-q_{0}\right|_{Q}+\int_{0}^{t}\left|A_{0}^{\alpha} T_{0}(t-s)\left\{B\left(u^{n}(s)\right)\left\{q^{n}(s)-q(s)\right\}+B(u(s))\{\bar{q}-q(s)\}\right\}\right| \\
& \quad+e^{-\lambda(t-s)} \mid \lambda\left\{q^{n}(s)-q(s)\right\}+B(u(s))^{\prime}\{u(s)-v(s)\} \\
& \quad-\left.P_{Q}^{n} B\left(u^{n}(s)\right)^{\prime}\left\{u^{n}(s)-v^{n}(s)\right\}\right|_{Q} d s \\
& \leq\left|P_{Q}^{n} q_{0}-q_{0}\right|_{Q}+\int_{0}^{t} M_{\alpha}(t-s)^{-\alpha}\left\{\gamma_{1}\left|\bar{q}_{Q}\right| u^{n}(s)-\left.u(s)\right|_{\alpha}+\gamma_{1}\left|q^{n}(s)\right|_{Q}\left|u^{n}(s)-u(s)\right|_{\alpha}\right. \\
& \left.+\left.\beta_{1}\left|q^{n}(s)-q(s)\right|\right|_{Q}|u(s)|_{\alpha}\right\}+\lambda\left|q^{n}(s)-q(s)\right|_{Q}+\gamma_{1}\left|u^{n}(s)-u(s)\right|_{\alpha}\left|u^{n}(s)\right| \\
& +\gamma_{1}\left|u^{n}(s)-u(s)\right||u(s)|_{\alpha}+\gamma_{1}\left|u^{n}(s)-u(s)\right|_{\alpha}\left|v^{n}(s)\right| \\
& +\gamma_{1}|u(s)|_{\alpha}\left|v^{n}(s)-v(s)\right|+\left|\left\{I-P_{Q}^{n}\right\} B(u(s))^{\prime}\{u(s)-v(s)\}\right|_{Q} d s,
\end{aligned}
$$

where in the above estimate we have invoked Assumptions (A7) and (A8). Assumption (A14), Corollary 4.4, (2.56) and (4.39) then imply that

$$
\left|\hat{x}^{n}(t)-\hat{x}(t)\right|_{\hat{X}_{\alpha}} \leq \varepsilon^{n}+\kappa \int_{0}^{t}(t-s)^{-\alpha}\left|\hat{x}^{n}(s)-\hat{x}(s)\right|_{\hat{X}_{\alpha}} d s,
$$

where $\kappa>0$ and $\lim _{n \rightarrow \infty} \varepsilon^{n}=0$. The estimate given in (4.46) together with a careful inspection of the proof of Lemma 5.6.7 in [32] then yield that

$$
\left|\hat{x}^{n}(t)-\hat{x}(t)\right|_{\hat{X}_{\alpha}} \leq \varepsilon^{n} K_{T}, \quad 0 \leq t \leq T,
$$

where $K_{T}=\frac{T^{1-\alpha}}{1-\alpha}>0$. This establishes (4.40) and (4.41).

We now turn our attention to establishing (4.43). For $t \in[0, T]$ and $\varphi \in V$. Assumptions $(A 2),(A 3),(A 6),(A 7)$ and $(A 8),(2.1),(4.42)$ and Lemma 4.6 (or, equivalently, (4.44)) imply that

$$
\begin{aligned}
& \left|\left\langle f^{n}(t)-f(t), \varphi\right\rangle\right| \\
& =\left|\left\langle A\left(q^{n}(t)\right) u^{n}(t)-A_{0} u^{n}(t)-A(q(t)) u(t)+A_{0} u(t), \varphi\right\rangle\right| \\
& \leq\left|\left\langle A_{1}\left(q^{n}(t)\right) u^{n}(t)-A_{1}(q(t)) u^{n}(t), \varphi\right\rangle\right|+\left|\left\langle A_{1}(q(t)) u^{n}(t)-A_{1}(q(t)) u(t), \varphi\right\rangle\right| \\
& \quad+\left|\left\langle A_{2} u^{n}(t)-A_{2} u(t), \varphi\right\rangle\right|+\left|\left\langle A_{0} u^{n}(t)-A_{0} u(t), \varphi\right\rangle\right| \\
& \leq K \beta_{1}\left|q^{n}(t)-q(t)\right|_{Q}\left|u^{n}(t)\right|_{\alpha}\|\varphi\|+K \gamma_{1}|q(t)|_{Q}\left|u^{n}(t)-u(t)\right|_{\alpha}\|\varphi\| \\
& \quad+\left\|A_{2} u^{n}(t)-\left.A_{2} u(t)\right|_{*}|| \varphi\right\|+\alpha_{0}|| u^{n}(t)-u(t)\|\| \varphi \| \\
& \leq K \beta_{1}\left|q^{n}(t)-q(t)\right|_{Q}\left|u^{n}(t)\right|_{\alpha}\|\varphi\|+K \gamma_{1}|q(t)|_{Q}\left|u^{n}(t)-u(t)\right|_{\alpha}\|\varphi\| \\
& \quad+\gamma_{2}\left|u^{n}(t)-u(t)\right|_{\alpha}\|\varphi\|+\alpha_{0} K_{V}\left|u^{n}(t)-u(t)\right|_{\alpha}\|\varphi\| .
\end{aligned}
$$

Recalling (2.56), (4.39), (4.40), and (4.41), we obtain

$$
\lim _{n \rightarrow \infty}\left\|f^{n}(t)-f(t)\right\|_{*}=0,
$$

uniformly in $t$ for $t \in[0, T]$, and the theorem is proved. $\square$ 


\section{$5 \quad$ Examples and Numerical Results}

In this section we describe and discuss four different examples which illustrate the application of the general theory developed in the previous sections. We consider the example involving a first order hyperbolic plant and a diffusion equation reference model discussed in Section 2.1, an example involving the identification of a spatially varying thermal conductivity in a heat equation plant, an example involving the identification of a damped wave equation, and an example in which we identify the nonlinearity in a quasi-linear heat equation. All of the computations to be described below were carried out via codes written in Fortran and run on either a SUN SPARCstation 10 in the Department of Mathematics at the University of Southern California, or an IBM RISCSystem 6000 at the Center for Research in Scientific Computation at North Carolina State University. The closed loop system (2.27)-(2.30) was discretized using a spline based Galerkin scheme. The resulting finite dimensional system of nonlinear ordinary differential equations were integrated using either the stiff ODE solver from the NAG Library, routine D02NBF (at USC), or a fourth order Runge-Kutta scheme (at NCSU). All required integrals were computed numerically via a composite two point Gauss-Legendre quadrature rule.

Example 5.1 We consider the example discussed in Section 2.1. In particular, we use this example to illustrate the approximation results obtained in Section 4. Recall from Section 2.1 that $H=L_{2}(0,1), V=H_{L}^{1}(0,1)=\left\{\varphi \in H^{1}(0,1): \varphi(0)=0\right\}, \hat{V}^{*}=V^{*}$, and $Q=\mathbf{R}^{1}$. The inner product on $Q$ was chosen to be $\langle q, p\rangle_{Q}=\omega q \cdot p$ for $q, p \in \mathbf{R}^{1}$. The weighting factor $\omega>0$ serves as an adaptive gain which can be used to tune the estimator. The plant is given by

$$
\begin{gathered}
\frac{\partial u}{\partial t}(t, x)+\bar{q} \frac{\partial u}{\partial x}(t, x)=f(t, x), \quad 0<x<1, t>0, \\
u(t, 0)=0, \quad t>0, \\
u(0, x)=u_{0}(x), \quad 0 \leq x \leq 1,
\end{gathered}
$$

where $\bar{q}>0, u_{0} \in L_{2}(0,1)$, and $t \rightarrow f(t, \cdot) \in L_{2}(0, T ; H)$, for each $T>0$. The reference model is given by

$$
\begin{gathered}
\frac{\partial v}{\partial t}(t, x)-a_{0} \frac{\partial^{2} v}{\partial x^{2}}(t, x)=g(t, x) \quad 0<x<1, t>0, \\
v(t, 0)=0, \quad \text { and } \quad \frac{\partial v}{\partial x}(t, 1)=0, \quad t>0, \\
v(0, x)=v_{0}(x), \quad 0 \leq x \leq 1,
\end{gathered}
$$

where $a_{0}>0, v_{0} \in L_{2}(0,1)$, and $t \rightarrow g(t, \cdot) \in L_{2}\left(0, T ; V^{*}\right)$, for each $T>0$.

We approximate using linear B-splines. For $n=1,2, \ldots$, let $\left\{\varphi_{j}^{n}\right\}_{j=0}^{n}$ be the standard linear B-splines on the interval $[0,1]$ defined with respect to the uniform mesh $\left\{0, \frac{1}{n}, \frac{2}{n}, \ldots, 1\right\}$. That is, for $i=0,1,2, \ldots, n$

$$
\varphi_{i}^{n}(x)=\left\{\begin{array}{cc}
1-|n x-i|, & x \in\left[\frac{i-1}{n}, \frac{i+1}{n}\right], \\
0, & \text { elsewhere on }[0,1] .
\end{array}\right.
$$

Set $H^{n}=\operatorname{span}\left\{\varphi_{j}^{n}\right\}_{j=1}^{n} \subset V$. Since $Q$ is finite dimensional, we simply set $Q^{n}=Q=\mathbf{R}^{1}$, $n=1,2, \ldots$. For each $n=1,2, \ldots$, let $P^{n}$ denote the orthogonal projection of $H$ onto $H^{n}$ and 
setting $\pi_{n}=P^{n}$, standard approximation results for spline functions (see [35]) can be used to establish that Assumption ( $A 13$ ) is satisfied. Thus the conclusions of Theorem 4.7 hold.

We set $\bar{q}=1.0, a_{0}=.1, \omega=.02$, and $q_{0}=0.0$. We also set

$$
u_{0}(x)=0.0, \quad \text { and } \quad v_{0}(x)=\sin \left(\frac{\pi}{2} x\right), \quad 0 \leq x \leq 1,
$$

and

$$
g(t, x)=5 \sin \left(\frac{\pi}{2} t\right) \chi_{[.215, .315]}(x), \quad 0<x<1, t>0 .
$$

We simulated the plant using a 64 linear spline based Galerkin scheme, and approximated the reference model in $H^{n}$ with $n=8,16$ and 32. In Figure 5.1a we have plotted the parameter estimator trajectories, $q^{n}(t)$, along with the trajectory of the infinite dimensional estimator (i.e. $n=64), q(t)$, for $0 \leq t \leq 100$. In Figure $5.1 \mathrm{~b}$ we plot the $L_{2}$ norms of the corresponding state tracking errors, $\left|e^{n}(t)\right|=\left|u(t)-v^{n}(t)\right|$, for $0 \leq t \leq 100$. It is clear from the figures that the scheme performed well for $n$ as small as 8 . We note that the scheme even performed reasonably well for $n=4$, although we have not plotted these results here.

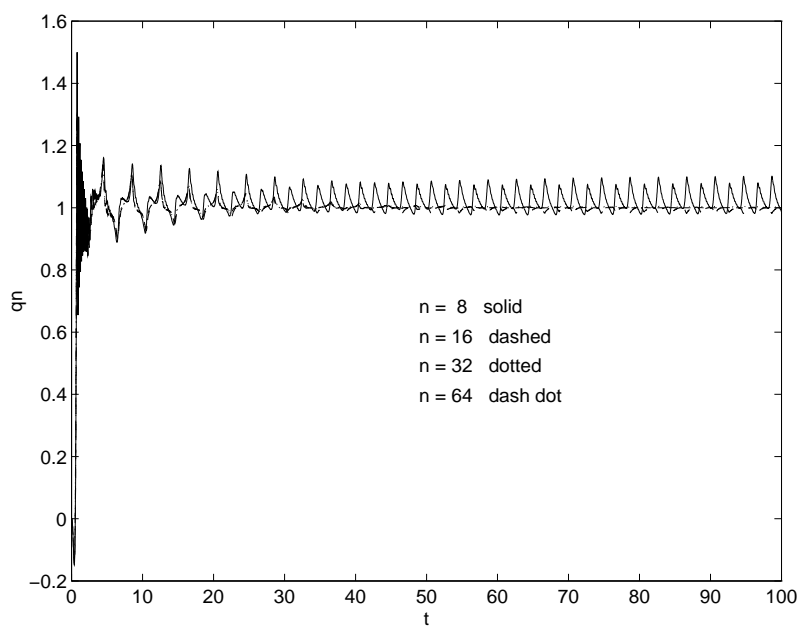

(a)

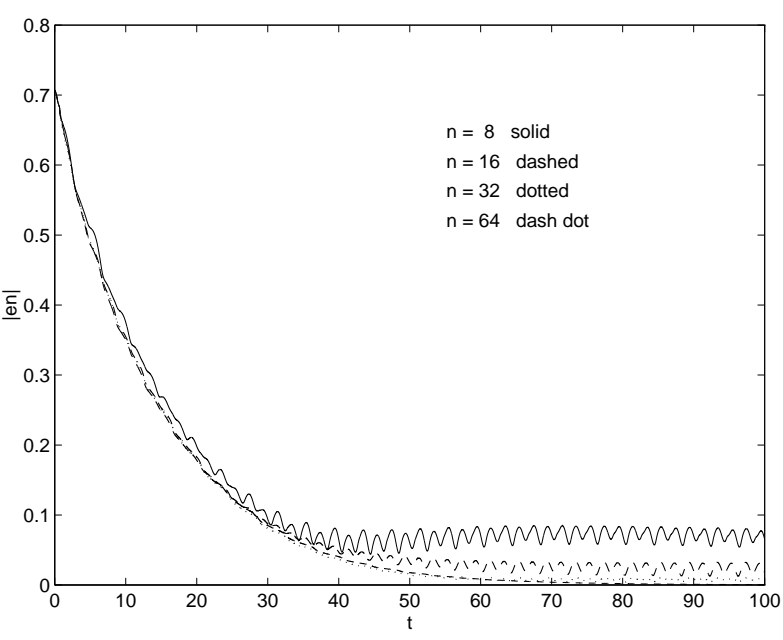

(b)

Figure 5.1: Results for Example 5.1: (a) the parameter estimates, (b) the tracking error.

Example 5.2 In this example we consider the control of the one dimensional heat or diffusion equation

$$
\frac{\partial u}{\partial t}(t, x)=\frac{\partial}{\partial x}\left\{\bar{q}(x) \frac{\partial u}{\partial x}(t, x)\right\}+f(t, x), \quad t>0, \quad 0<x<1,
$$

together with the Dirichlet boundary conditions

$$
u(t, 0)=0=u(t, 1), \quad t>0 .
$$

We take the reference model to be given by

$$
\begin{gathered}
\frac{\partial v}{\partial t}(t, x)-a_{0} \frac{\partial^{2} v}{\partial x^{2}}(t, x)=g(t, x) \quad 0<x<1, t>0, \\
v(t, 0)=0, \quad \text { and } \quad v(t, 1)=0, \quad t>0, \\
v(0, x)=v_{0}(x), \quad 0 \leq x \leq 1,
\end{gathered}
$$


where $a_{0}>0, v_{0} \in L_{2}(0,1)$, and $t \rightarrow g(t, \cdot) \in L_{2}\left(0, T ; V^{*}\right)$, for each $T>0$.

In this case we have $H=L_{2}(0,1)$ and $V=H_{0}^{1}(0,1)$, each endowed with its usual inner product and corresponding induced norm. We set $\hat{V}^{*}=V^{*}$, and we let $Q=H^{1}(0,1)$ and take it to be endowed with the weighted inner product

$$
\langle q, p\rangle_{Q}=\omega_{1} \int_{0}^{1} q(x) p(x) d x+\omega_{2} \int_{0}^{1} D q(x) D p(x) d x, \quad p, q \in H^{1}(0,1),
$$

where the weights $\omega_{1}$ and $\omega_{2}$, assumed to be positive, serve as adaptive gains, or tuning parameters. For $q \in Q$, the operator $A(q)=A_{1}(q) \in \mathcal{L}\left(V, V^{*}\right)$ is given by

$$
\langle A(q) \varphi, \psi\rangle=\left\langle A_{1}(q) \varphi, \psi\right\rangle=\int_{0}^{1} q(x) D \varphi(x) D \psi(x) d x, \quad \varphi, \psi \in H^{1}(0,1) .
$$

The operator $A_{0} \in \mathcal{L}\left(V, V^{*}\right)$ given by

$$
\left\langle A_{0} \varphi, \psi\right\rangle=a_{0} \int_{0}^{1} D \varphi(x) D \psi(x) d x, \quad \varphi, \psi \in H_{0}^{1}(0,1) .
$$

It is easily verified that Assumptions $(A 1)-(A 5)$ are satisfied, and that the theory in Section 2.2 applies.

To simulate the closed loop system, we discretized equations (2.27)-(2.30) using a linear spline based Galerkin scheme. We approximated the plant and reference model state space $H$ by $H^{n}=\operatorname{span}\left\{\varphi_{j}^{n}\right\}_{j=1}^{n-1}$, where the linear B-splines, $\varphi_{j}^{n}$, are given by (5.1). We also used linear B-splines to discretize the parameter space $Q$. We set $Q^{m}=\operatorname{span}\left\{\varphi_{j}^{m}\right\}_{j=0}^{m}$, where the linear spline basis, $\left\{\varphi_{j}^{m}\right\}_{j=0}^{m}$ is again given by (5.1) with $n$ replaced by $m$. Note that dim $H^{n}=n-1$ and $\operatorname{dim} Q^{m}=m+1$. Consequently the dimension of the approximating estimator is $n-1+m+1=n+m$.

We set $a_{0}=0.1, \omega_{1}=0.1, \omega_{2}=0.0001$,

$$
\bar{q}(x)=\frac{1}{10}\left\{1-\frac{1}{2} \sin \left(2 \pi\left\{x-\frac{1}{4}\right\}\right)\right\}, \quad 0<x<1,
$$

$q_{0}(x)=0.1, u_{0}(x)=0.3(0.5-|0.5-x|)$ and $v_{0}(x)=-0.1 \sin (\pi x)$, for $0 \leq x \leq 1$. We chose the input reference signal, $g$, to be given by

$$
g(t, x)=.1\left\{\sin \left(\frac{\pi}{24} t\right)+\cos \left(\frac{\pi}{50} t\right)+\frac{1}{2} \cos \left(\frac{\pi}{30} t\right)\right\} \chi_{[.4,6]}(x), \quad 0<x<1, t>0 .
$$

The results of our numerical study with $n=24$ and $m=16$ are displayed in Figures 5.2a and $5.2 \mathrm{~b}$. In Figure 5.2a we have plotted $q_{0}, \bar{q}$, and the estimate for $\bar{q}, q(t)$, at $t=25$. In Figure $5.2 \mathrm{~b}$ we have plotted the $L_{2}$ norm of the state tracking error, $|e(t)|=|u(t)-v(t)|$ for $0 \leq t \leq 50$. It is clear that the control objective has been met, and that an excellent estimate for $\bar{q}$ has been obtained. 


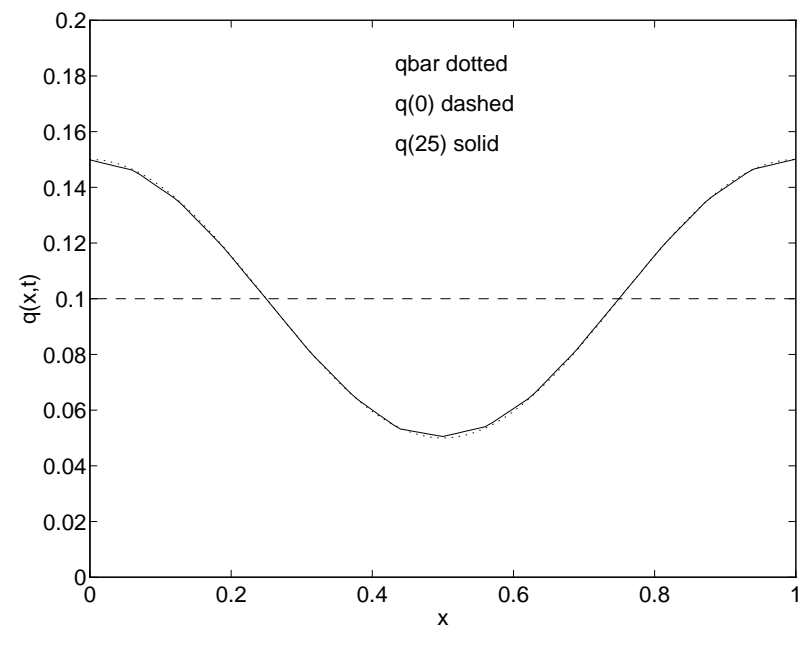

(a)

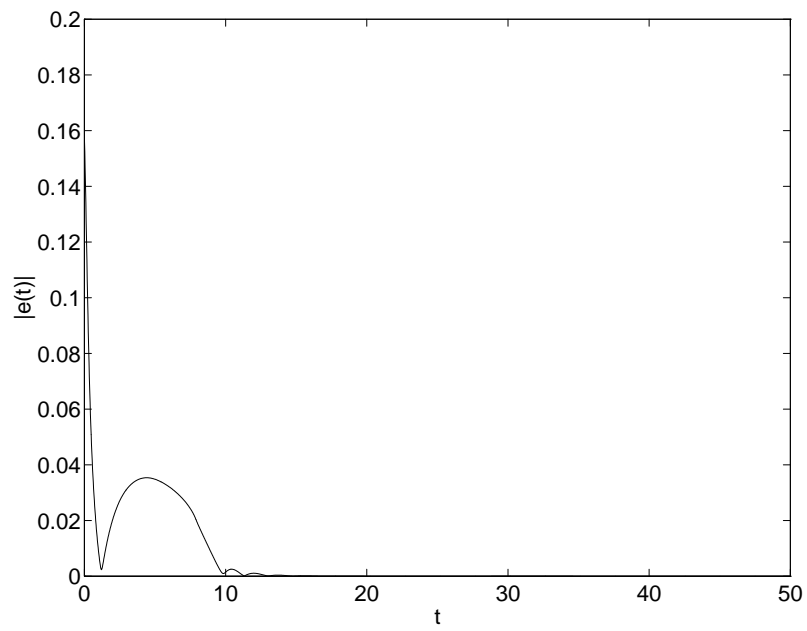

(b)

Figure 5.2: Results for Example 5.2: (a) the parameter estimate, (b) the tracking error.

Example 5.3 In this example we consider the control of the one dimensional wave equation with Kelvin - Voigt viscoelastic damping given by

$$
\frac{\partial^{2} u_{1}}{\partial t^{2}}(t, x)-\bar{q}_{2} \frac{\partial^{2}}{\partial x^{2}} \frac{\partial u_{1}}{\partial t}(t, x)-\bar{q}_{1} \frac{\partial^{2} u_{1}}{\partial x^{2}}=f_{1}(t, x), 0<x<1, t>0,
$$

with the Dirichlet (fixed endpoint) boundary conditions

$$
u_{1}(t, 0)=0=u_{1}(t, 1), \quad t>0,
$$

and the initial conditions

$$
u_{1}(0, x)=u_{01}(x), \quad \text { and } \quad \frac{\partial u_{1}}{\partial t}(0, x)=u_{02}(x), \quad 0 \leq x \leq 1 .
$$

We take the reference model to be given by

$$
\begin{gathered}
\frac{\partial^{2} v_{1}}{\partial t^{2}}(t, x)-b_{0} \frac{\partial^{2}}{\partial x^{2}} \frac{\partial v_{1}}{\partial t}(t, x)-a_{0} \frac{\partial^{2} v_{1}}{\partial x^{2}}=g_{1}(t, x), 0<x<1, t>0, \\
v_{1}(t, 0)=0=v_{1}(t, 1), \quad t>0, \\
v_{1}(0, x)=v_{01}(x), \quad \text { and } \quad \frac{\partial v_{1}}{\partial t}(0, x)=v_{02}(x), \quad 0 \leq x \leq 1,
\end{gathered}
$$

where $a_{0}, b_{0}>0$. To apply our theory in this case, requires rewriting the plant and reference model as equivalent first order systems. Let $H_{1}=L_{2}(0,1)$ be endowed with the standard inner product and corresponding induced norm denoted by $\langle\cdot, \cdot\rangle_{1}$ and $|\cdot|_{1}$, respectively. Let $V_{1}=H_{0}^{1}(0,1)$, be endowed with the inner product (and corresponding induced norm) given by $[\varphi, \psi]_{1}=\langle D \varphi, D \psi\rangle_{1}$, for $\varphi, \psi \in V_{1}$. Let $H=V_{1} \times H_{1}$ and $V=V_{1} \times V_{1}$. We endow $V$ with the usual product inner product, but we endow $H$ with the inner product given by

$$
\langle\varphi, \psi\rangle=\gamma\left\{a_{0}\left[\varphi_{1}, \psi_{1}\right]_{1}+\left\langle\varphi_{2}, \psi_{2}\right\rangle_{1}\right\}+\left\langle\varphi_{1}, \psi_{2}\right\rangle_{1}+\left\langle\varphi_{2}, \psi_{1}\right\rangle_{1}+b_{0}\left[\varphi_{1}, \psi_{1}\right]_{1},
$$


for $\varphi=\left(\varphi_{1}, \varphi_{2}\right), \psi=\left(\psi_{1}, \psi_{2}\right) \in H$, where $\gamma>0$. It is not difficult to argue that if $\gamma>$ $\max \left\{1, a_{0}^{-1}, b_{0}^{-1}\right\}$, then the norm induced by this inner product is equivalent to the standard norm on $H$. The inner product on $H$ is chosen in this way so that Assumption (A4) will be satisfied by the operator $A_{0}$ to be defined below. We note that this choice of an inner product affects the form of the estimator equation, (2.25). Thus in practice, $\gamma$ serves as an additional tuning parameter or adaptive gain.

We let $Q=\mathbf{R}^{2}$ with the weighted inner product given by

$$
\langle q, p\rangle=q^{T} \Omega p, \quad q, p \in \mathbf{R}^{2},
$$

where $\Omega$ is the $2 \times 2$ diagonal matrix given by

$$
\Omega=\left[\begin{array}{cc}
\omega_{1} & 0 \\
0 & \omega_{2}
\end{array}\right],
$$

with $\omega_{1}, \omega_{2}>0$.

For $q=\left(q_{1}, q_{2}\right)^{T} \in Q$, we define the operator $A(q) \in \mathcal{L}(V, V)$ by $A(q)=A_{1}(q)+A_{2}$, where

$$
\left\langle A_{1}(q) \varphi, \psi\right\rangle=q_{2}\left\langle D \varphi_{2}, D \psi_{2}\right\rangle+q_{1}\left\langle D \varphi_{1}, D \psi_{2}\right\rangle, \quad \varphi=\left(\varphi_{1}, \varphi_{2}\right), \psi=\left(\psi_{1}, \psi_{2}\right) \in V,
$$

and

$$
\left\langle A_{2} \varphi, \psi\right\rangle=-a_{0}\left\langle D \varphi_{2}, D \psi_{1}\right\rangle, \quad \varphi=\left(\varphi_{1}, \varphi_{2}\right), \psi=\left(\psi_{1}, \psi_{2}\right) \in V .
$$

We take the operator $A_{0} \in \mathcal{L}\left(V, V^{*}\right)$ to be given by $A_{0}=A\left(q^{*}\right)$, where $q^{*}=\left(a_{0}, b_{0}\right) \in Q$. We set $f=\left(0, f_{1}\right), g=\left(0, g_{1}\right), u_{0}=\left(u_{01}, u_{02}\right)$, and $v=\left(v_{01}, v_{02}\right)$. Thus we have rewritten the plant, (5.6)-(5.8), in the form (2.5), (2.6), and the reference model, (5.9)-(5.11), in the form (2.7), (2.8), with $u=\left(u_{1}, D_{t} u_{1}\right)$ and $v=\left(v_{1}, D_{t} v_{1}\right)$. It can be verified that Assumptions $(A 1)$ - $(A 5)$ are satisfied with $\hat{V}^{*}=\left\{(0, \varphi): \varphi \in H^{-1}(0,1)\right\} \subset V^{*}=H_{0}^{1}(0,1) \times H^{-1}(0,1)$.

To simulate the closed loop system, we again approximate using the linear spline basis given in (5.1) and a Galerkin scheme. We set $H_{1}^{n}=\operatorname{span}\left\{\varphi_{j}^{n}\right\}_{j=1}^{n-1}$ and set $H^{n}=H_{1}^{n} \times H_{1}^{n}$. We took $\bar{q}=(0.0308,0.01), q^{*}=\left(a_{0}, b_{0}\right)=(0.0056,0.0028)$, and $q_{0}=(0.02,0.005)$. We set

$$
\begin{gathered}
u_{01}(x)=0.01 \sin (\pi x) \quad \text { and } \quad u_{02}(x)=0.001 \sin (4 \pi x), \quad 0 \leq x \leq 1, \\
v_{01}(x)=0 \quad \text { and } \quad v_{02}(x)=0, \quad 0 \leq x \leq 1,
\end{gathered}
$$

and

$$
g_{1}(t, x)=\{4 \sin (4 \pi t)+\cos (\pi t)+2\} \chi_{[.215,315]}(x), \quad t>0,0<x<1 .
$$

We chose the adaptive gains to be $\omega_{1}=\omega_{2}=1600 / 3$ and $\gamma=100+1 / b_{0}=457.15$. We then simulated the closed loop system with $n=16$. In Figure 5.3 we have plotted the estimate for $\bar{q}_{1}$ $q_{1}(t)$, and the estimate for $\bar{q}_{2}, q_{2}(t)$, for $t \in[0,20]$. In Figure 5.4 we have plotted the $V_{1}$-norm of the displacement tracking error, $\|e(t)\|_{1}=\left\|u_{1}(t)-v_{1}(t)\right\|_{1}$, and the $H_{1}$-norm of the velocity tracking error, $\left|D_{t} \epsilon(t)\right|_{1}=\left|D_{t} u_{1}(t)-D_{t} v_{1}(t)\right|_{1}$, for $t \in[0,100]$. 


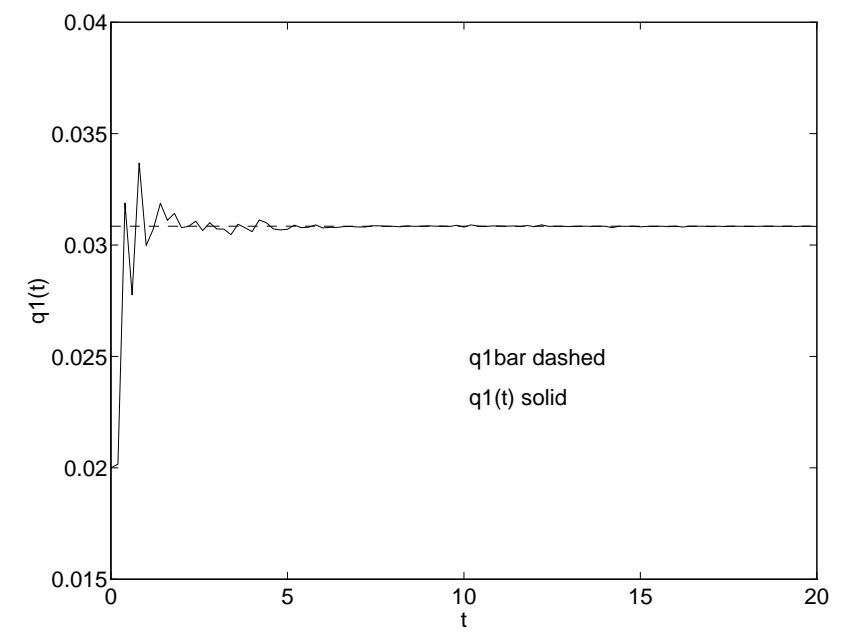

(a)

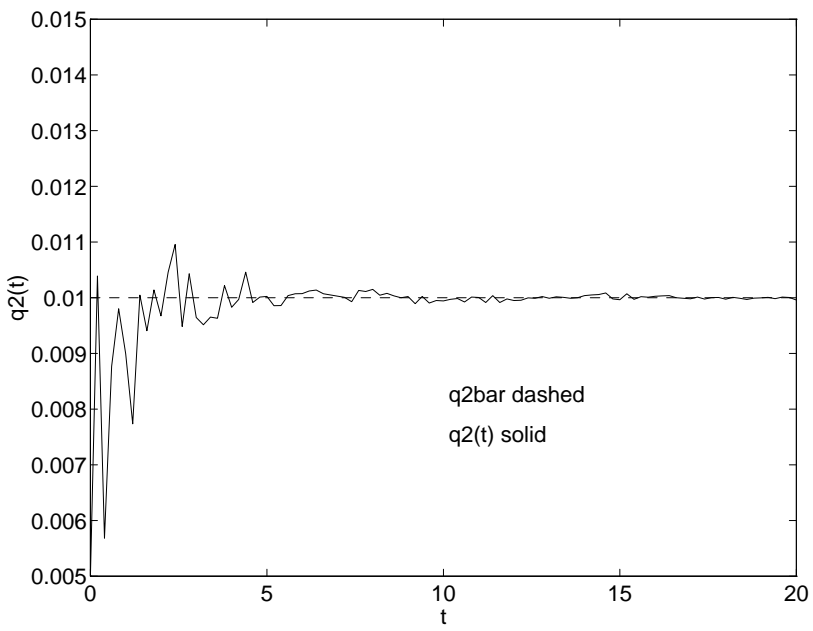

(b)

Figure 5.3: Results for Example 5.3: (a) the estimate for $\bar{q}_{1}$, (b) the estimate for $\bar{q}_{2}$.

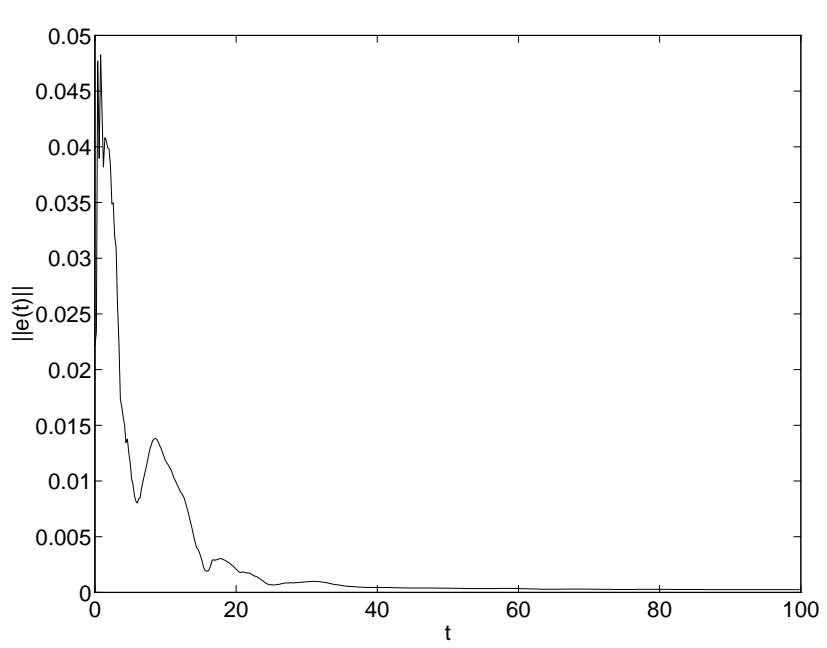

(a)

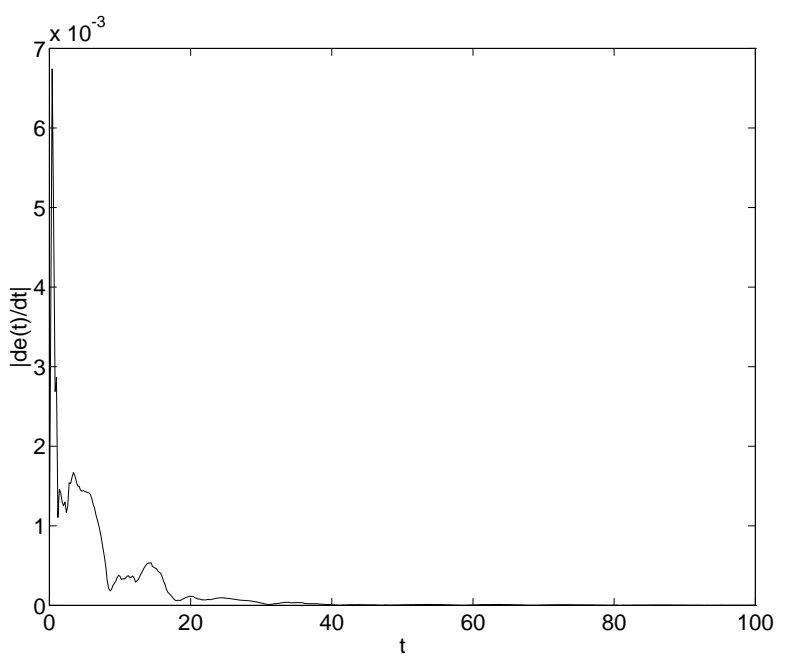

(b)

Figure 5.4: Results for Example 5.3: (a) the $V_{1}$-norm of the displacement tracking error, (b) the $H_{1}$-norm of the velocity tracking error.

Example 5.4 In this example we consider the control of the one dimensional nonlinear (strictly speaking, quasi-linear) heat equation

$$
\frac{\partial u}{\partial t}(t, x)-\frac{\partial}{\partial x}\left\{\bar{q}\left(\min \left\{M,\left|\frac{\partial u}{\partial x}(t, x)\right|\right\}\right) \frac{\partial u}{\partial x}(t, x)\right\}=f(t, x), \quad 0<x<1, t>0,
$$

together with the Dirichlet boundary conditions

$$
u(t, 0)=0 \quad \text { and } \quad u(t, 1)=0, \quad t>0,
$$


and the initial conditions

$$
u(0, x)=u_{0}(x), \quad 0 \leq x \leq 1 .
$$

We assume that $M \in[0, \infty), u_{0} \in L_{2}(0,1)$ and $f(t, \cdot) \in L_{2}(0,1)$ for $t \geq 0$. We assume that the nonlinearity, $\bar{q}$, is unknown, and is to be identified as the system (5.12)-(5.13) is being adaptively controlled. Once again, we take the reference model to be given by (5.2)-(5.4).

We let $H=L_{2}(0,1)$ be endowed with the standard inner product, we let $V=H_{0}^{1}(0,1)$ be endowed with the usual norm, and define the Hilbert space $Q$ as follows. Let $\hat{Q}=H^{1}\left(\mathbf{R}^{+}\right)$and define the inner product, $\langle\cdot, \cdot\rangle_{Q}$, on $\hat{Q}$ by

$$
\langle q, p\rangle_{Q}=\int_{0}^{\infty} \omega_{0}(\theta) q(\theta) p(\theta) d \theta+\int_{0}^{\infty} \omega_{1}(\theta) D q(\theta) D p(\theta) d \theta, \quad q, p \in \hat{Q},
$$

where $\omega_{0}, \omega_{1} \in L_{1}\left(\mathbf{R}^{+}\right)$are positive weighting functions. Let $|\cdot|_{Q}$ denote the norm induced by the inner product given in (5.15), and define the Hilbert space $Q$ to be the completion of the inner product space $\left\{\hat{Q},\langle\cdot, \cdot\rangle_{Q},|\cdot|_{Q}\right\}$. For $q \in Q$, the operator $A(q): V \rightarrow V^{*}$ is given by $A(q)=A_{1}(q)$, where $A_{1}(q): V \rightarrow V^{*}$ is defined by

$$
\left\langle A_{1}(q) \varphi, \psi\right\rangle=\int_{0}^{1} q(\min \{M,|D \varphi(x)|\}) D \varphi(x) D \psi(x) d x, \quad \varphi, \psi \in V .
$$

The operator $A_{0} \in \mathcal{L}\left(V, V^{*}\right)$ is once again given by (5.5). We set $\hat{V}^{*}=V^{*}=H^{-1}(0,1)$. It is not difficult to verify that Assumptions (A1)- $(A 5)$ are satisfied.

To simulate the closed loop system, we again approximate the plant and reference model state space, $H$, and the parameter space, $Q$, using linear B-spline functions. We approximate $H$ by $H^{n}=\operatorname{span}\left\{\varphi_{j}^{n}\right\}_{j=1}^{n-1}$, where for each $n=2,3, \ldots$ and $j=1,2, \ldots n-1, \varphi_{j}^{n}$ is given by (5.1). For each $m=1,2, \ldots$, and each $r>0$, let $\left\{\hat{\psi}_{j}^{m, r}\right\}_{j=0}^{m}$ be the standard linear B-splines on the interval $[0, r]$ defined with respect to the uniform mesh $\left\{0, \frac{r}{m}, \frac{2 r}{m}, \ldots, r\right\}$. We approximate $Q$ by $Q^{m, r}=\operatorname{span}\left\{\psi_{j}^{m, r}\right\}_{j=0}^{m}$, where

$$
\psi_{j}^{m, r}=\left\{\begin{array}{cc}
\hat{\psi}_{j}^{m, r}, & j=0,1,2, \ldots, m-1, \\
\hat{\psi}_{m}^{m, r}+\chi_{[r, \infty)}, & j=m,
\end{array}\right.
$$

with $\chi_{J}$ denoting the characteristic function for the interval $J$. In the simulations to be described below, only $q$ is discretized. The true value of $\bar{q}$,

$$
\bar{q}(\theta)=0.9\left(1-\frac{1}{2} \epsilon^{-\frac{1}{2} \theta^{2}}\right), \quad \theta \geq 0,
$$

is used. We chose $g$ to be given by

$$
\begin{aligned}
g(t, x)= & 1 \times 10^{-4}\{\sin (100 \pi t)+\sin (250 \pi t)+\sin (450 \pi t)+\sin (550 \pi t) \\
& +\sin (650 \pi t)+\sin (850 \pi t)+\cos (150 \pi t)+\cos (350 \pi t) \\
& +\cos (500 \pi t)+\cos (700 \pi t)\} \chi_{[0.6,0.8]}(x), \quad 0<x<1, \quad t>0,
\end{aligned}
$$

and set

$$
u_{0}(x)=5 \times 10^{-5} \quad \text { and } \quad v_{0}(x)=-0.1(0.5-|0.5-x|), \quad 0<x<1 .
$$

We set $a_{0}=.1, r=3.5, M=10.0$,

$$
\omega_{0}(\theta)=\omega_{1}(\theta)=\left\{\begin{array}{ll}
1 & 0 \leq \theta<r \\
\frac{1}{2} e^{-20 \theta} & r<\theta<\infty,
\end{array},\right.
$$


and

$$
q_{0}(\theta)=1, \quad 0<\theta<\infty .
$$

We simulated the closed loop system over the time interval $[0,20]$ using $n=32$ and $m=24$. In Figure 5.5a we have plotted our final (i.e. at time $t=20$ ) estimate for $\bar{q}$, and in Figure $5.5 b$ we have plotted the $H$-norm of the tracking error, $|e(t)|=|u(t)-v(t)|$, for $t \in[0,20]$. We note that convergence of the parameter estimates actually occured at about $t=5$. Our estimate for $\bar{q}$ in this example is not quite as good as the estimate obtained in Example 5.2. However, both the nonlinearity and the infinite domain of $\bar{q}$ (and therefore the need for an additional degree of approximation in the form of truncation) contribute to making this example a far more significant challenge for our scheme than the linear example discussed in Example 5.2.

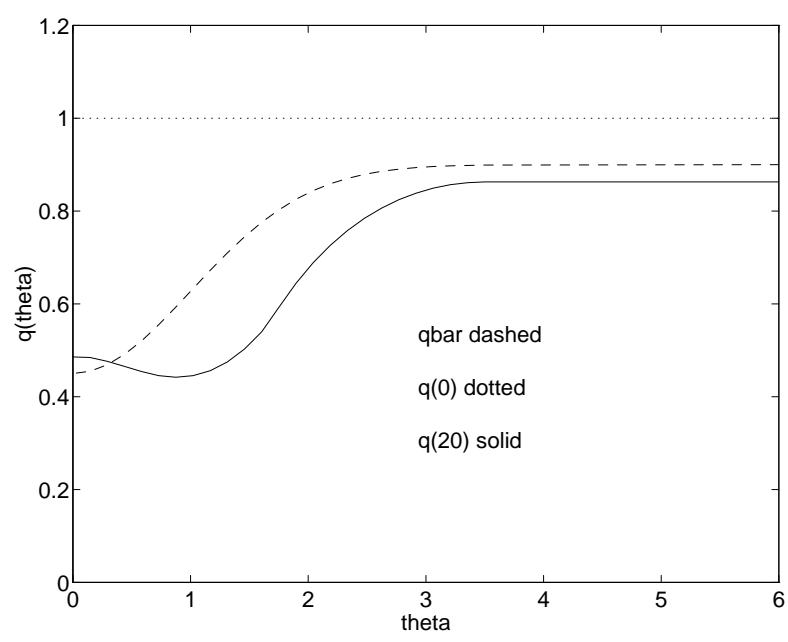

(a)

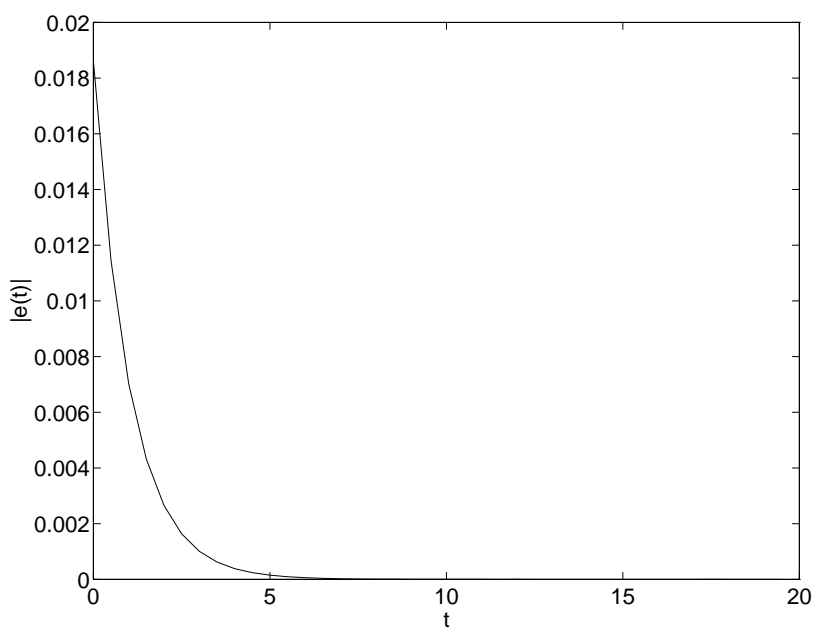

(b)

Figure 5.5: Results for Example 5.4: (a) the parameter estimate, (b) the tracking error. 


\section{References}

[1] H. W. Alt, K. H. Hoffmann, and J. Sprekels, A numerical procedure to solve certain identification problems, Intern. Series Numer. Math, 68 (1984), pp. 11-43.

[2] K. J. Astrom and B. Wittenmark, Adaptive Control, Addison-Wesley, Reading, MA, 1989.

[3] H. T. BANKS AND K. ITO, A unified framework for approximation and inverse problems for distributed parameter systems, Control Theory and Advanced Technology, 4 (1988), pp. 73-90.

[4] V. Barbu, Nonlinear Semigroups and Differential Equations in Banach Spaces, Noordhoff International Publishing, Leyden, The Netherlands, 1976.

[5] J. Baumeister and W. Scondo, Asymptotic embedding methods for parameter estimation, Proceedings of the 26th IEEE Conference on Decision and Control, (1987), pp. 170174.

[6] J. Baumeister, W. Scondo, M. A. Demetriou, and I. G. Rosen, On-line parameter estimation for infinite dimensional dynamical systems, SIAM Journal on Control and Optimization, (submitted).

[7] M. A. Demetriou and I. G. Rosen, Adaptive identification of second order distributed parameter systems, Inverse Problems, 10 (1994), pp. 261-294.

$[8]$ - On the persistence of excitation in the adaptive estimation of distributed parameter systems, IEEE Transactions on Automatic Control, 39 (1994), pp. 1117-1123.

[9] J. Dieudonné, Foundations of Modern Analysis, Academic Press, New York, 1960.

[10] T. E. Duncan and B. Pasik-Duncan, Adaptive control of linear delay time systems, Stochastics, 24 (1988), pp. 45-74.

[11] T. E. Duncan, B. Pasik-Duncan, and B. Goldys, Adaptive control of linear stochastic evolution systems, Stochastics and Stochastics Reports, 36 (1991), pp. 71-90.

[12] T. E. Duncan, B. Pasik-Duncan, and B. Maslowski, Some aspects of the adaptive boundary and point control of linear distributed parameter systems, Proceedings of the 31st IEEE Conference on Decision and Control, (1992), pp. 1077-1081.

[13] G. C. Goodwin And K. S. Sin, Adaptive Filtering Prediction and Control, Prentice-Hall, Englewood Cliffs, NJ, 1984.

[14] J. K. Hale, Ordinary Differential Equations, Wiley-Interscience, John Wiley and Sons, New York, 1969.

[15] K. H. Hoffmann and J. SpRekels, The method of asymptotic regularization and restricted parameter identification problems in variational inequalities, in Free Boundary Problems: Application and Theory, IV, Maubuisson, (1984), pp. 508-513. 
[16] - On the identification of coefficients of elliptic problems by asymptotic regularization, Numer. Funct. Anal. and Optimiz., 7 (1984-85), pp. 157-177.

[17] - On the identification of parameters in general variational inequalities by asymptotic regularization, SIAM J. Math. Anal., 17 (1986), pp. 1198-1217.

[18] K. S. Hong and J. Bentsman, Direct adaptive control of parabolic systems: Algorithm synthesis, and convergence and stability analysis, IEEE Transactions on Automatic Control, 39 (1994), pp. 2018-2033.

[19] T. Kato, Perturbation Theory for Linear Operators, Second Edition, Springer-Verlag, New York, 1984.

[20] T. Kobayashi, A digital adaptive control law for a parabolic distributed parameter system, Systems and Control Letters, 4 (1984), pp. 175-179.

[21] — Adaptive control for infinite dimensional systems, International Journal Systems Science, 17 (1986), pp. 887-896.

$[22]-$ Global adaptive stabilization of infinite dimensional systems, Systems and Control Letters, 9 (1987), pp. 215-223.

[23] - Model reference adaptive control for spectral systems, International Journal of Control, 46 (1987), pp. 1511-1523.

[24] —. Finite dimensional adaptive control for infinite dimensional systems, International Journal of Control, 48 (1988), pp. 289-302.

[25] S. G. Kreŭn, Linear Differential Equations in Banach Space, American Mathematical Society, Providence, 1971.

[26] J. L. Lions, Optimal Control of Systems Governed by Partial Differential Equations, Springer-Verlag, New York, 1971.

[27] J. L. Lions and E. Magenes, Problèmes aux Limites Non Homogènes et Applications, Volume 1, Dunod, Paris, 1968.

[28] A. P. Morgan and K. S. Narendra, On the stability of nonautonomous differential equations $\dot{x}=[A+B(t)]$, with skew symmetric matrix $B(t)$, SIAM J. Control and Optimization, 15 (1977), pp. 163-176.

[29] K. S. Narendra and A. M. Annaswamy, Stable Adaptive Systems, Prentice Hall, Englewood Cliffs, NJ, 1989.

[30] K. S. NARENDRA AND P. Kudva, Stable adaptive schemes for system identification and control, parts I and II, IEEE Trans. Systems, Man and Cybernetics, SMC-4 (1974), pp. 542560 .

[31] B. PASIK-DunCAN, On the consistency of a least squares identification procedure in linear evolution systems, Stochastics and Stochastics Reports, 39 (1992), pp. 83-94. 
[32] A. PAZY, Semigroups of Linear Operators and Applications to Partial Differential Equations, Springer-Verlag, New York, 1983.

[33] V. M. Popov, Hyperstability of Control Systems, Springer-Verlag, Berlin, 1973.

[34] S. Sastry and M. Bodson, Adaptive Control: Stability, Convergence and Robustness, Prentice-Hall, Englewood Cliffs, NJ, 1989.

[35] M. H. Schultz, Spline Analysis, Prentice Hall, Englewood Clffis, N.J., 1973.

[36] W. Scondo, Ein Modellabgleichsverfahren zur adaptiven Parameteridentifikation in Evolutionsgleichungen, PhD thesis, Johann Wolfgang Goethe-Universitat zu Frankfurt am Main, Frankfurt am Main, Germany, 1987.

[37] R. E. Showatter, Hilbert Space Methods for Partial Differential Equations, Pitman, London, 1977.

[38] H. Tanabe, Equations of Evolution, Pitman, London, 1979.

[39] J. T. Wen, Direct Adaptive Control in Hilbert Space, PhD thesis, Electrical, Computer and Systems Engineering Department, Renselaer Polytechnic Institute, Troy, NY, 1985.

[40] J. T. WEn And M. J. BaLAs, Robust adaptive control in Hilbert space, Journal of Mathematical Analysis and Applications, 143 (1989), pp. 1-26. 